\title{
STABILITY AND STABILIZABILITY OF MIXED RETARDED-NEUTRAL TYPE SYSTEMS *
}

\author{
Rabah Rabah ${ }^{1}$, Grigory Mikhailovitch Sklyar ${ }^{2}$ \\ AND PAVEL YuREVITCH BARKHAYEV ${ }^{3}$
}

\begin{abstract}
We analyze the stability and stabilizability properties of mixed retarded-neutral type systems when the neutral term may be singular. We consider an operator differential equation model of the system in a Hilbert space, and we are interested in the critical case when there is a sequence of eigenvalues with real parts converging to zero. In this case, the system cannot be exponentially stable, and we study conditions under which it will be strongly stable. The behavior of spectra of mixed retarded-neutral type systems prevents the direct application of retarded system methods and the approach of pure neutral type systems for the analysis of stability. In this paper, two techniques are combined to obtain the conditions of asymptotic non-exponential stability: the existence of a Riesz basis of invariant finite-dimensional subspaces and the boundedness of the resolvent in some subspaces of a special decomposition of the state space. For unstable systems, the techniques introduced enable the concept of regular strong stabilizability for mixed retarded-neutral type systems to be analyzed.
\end{abstract}

Mathematics Subject Classification. 34K40, 34K20, 93C23, 93D15.

Received November 12, 2009. Revised December 17, 2010.

Published online September 19, 2011.

\section{INTRODUCTION}

The interest in delay differential equations and the corresponding infinite-dimensional systems stems from the fact that many systems in economics, science and engineering can be described by such equations. The stability theory of such systems has been studied intensively (see e.g. [3,10,15]). A number of results have been obtained for retarded systems, however an analysis of neutral type systems is much more complicated and these systems are thus less fully understood. We consider neutral type systems given by the following functional differential equation:

$$
\frac{\mathrm{d}}{\mathrm{d} t}\left[z(t)-K z_{t}\right]=L z_{t}+B u(t), \quad t \geq 0
$$

Keywords and phrases. Retarded-neutral type systems, asymptotic non-exponential stability, stabilizability, infinite dimensional systems.

* The work was partially supported by the Polish Ministry of Science and High Education grant No. N514 238438 and École Centrale de Nantes, France.

${ }^{1}$ IRCCyN/École des Mines de Nantes, 4 rue Alfred Kastler, BP 20722, 44307 Nantes Cedex 3, France. rabah@emn.fr

${ }^{2}$ Institute of Mathematics, University of Szczecin, Wielkopolska 15, 70-451 Szczecin, Poland. sklar@univ.szczecin.pl

${ }^{3}$ Dept. of Diff. Equat. and Control, Kharkov National University, 4 Svobody sqr., 61077 Kharkov, Ukraine. pbarhaev@inbox.ru 
where $z_{t}:[-1,0] \rightarrow \mathbb{C}^{n}$ is the history of $z$ defined by $z_{t}(\theta)=z(t+\theta)$. We assume the delay operator $L: H^{1}\left([-1,0], \mathbb{C}^{n}\right) \rightarrow \mathbb{C}^{n}$ to be linear and bounded, thus, it has the following form:

$$
L f=\int_{-1}^{0} A_{2}(\theta) f^{\prime}(\theta) \mathrm{d} \theta+\int_{-1}^{0} A_{3}(\theta) f(\theta) \mathrm{d} \theta, \quad f \in H^{1}\left([-1,0], \mathbb{C}^{n}\right),
$$

where $A_{2}, A_{3}$ are $n \times n$-matrices whose elements belong to $L_{2}([-1,0], \mathbb{C})$. We take the difference operator $K$ in the form

$$
K f=A_{-1} f(-1)
$$

where $A_{-1}$ is a constant $n \times n$-matrix. The form (1.3) may be considered as a particular case of the operator $K: C\left([-1,0], \mathbb{C}^{n}\right) \rightarrow \mathbb{C}^{n}$ given by $K f=\int_{-1}^{0} \mathrm{~d} \mu(\theta) f(\theta)$, where $\mu(\cdot):[-1,0] \rightarrow \mathbb{C}^{n \times n}$ is of bounded variation and continuous at zero. However, the analysis of the system (1) with $K$ as in (3) is difficult enough to warrant a study of its own. As we shall see, the results depend strongly on the properties of the matrix $A_{-1}$.

The well-known approach, when studying systems of the form (1.1), is to consider a corresponding infinitedimensional model $\dot{x}=\mathcal{A} x$, where $\mathcal{A}$ is the infinitesimal generator of a $C_{0}$-semigroup. For systems (1.1)-(1.3), the resolvent of the operator $\mathcal{A}$ allows an explicit representation (see $[27,28]$ ). Such a representation is an effective tool for analyzing the exponential stability property since the latter is equivalent to the uniform boundedness of the resolvent on the complex right half-plane. The resolvent boundedness approach is exhaustive when one considers the stability of pure retarded type systems $\left(A_{-1}=0\right)$ since such systems may be exponentially stable or unstable only. This is because the exponential growth of the semigroup $\left\{\mathrm{e}^{t \mathcal{A}}\right\}_{t \geq 0}$ is determined by the spectrum's location and there is only a finite number of eigenvalues of $\mathcal{A}$ in any half-plane $\{\lambda: \operatorname{Re} \lambda \geq C\}$.

For neutral-type systems $\left(A_{-1} \neq 0\right)$, in addition to the notion of exponential stability, which is characterized by the condition that the spectrum is bounded away from the imaginary axis (see [12, Thm. 6.1],[10]), we have the notion of strong asymptotic non-exponential stability. This type of stability may occur in some critical cases when the exponential stability is not possible (see e.g. [6]). Thus, strong stability cannot be described in terms of the resolvent boundedness. In [27,28], for neutral type systems with a non-singular neutral term (det $A_{-1} \neq 0$ ), this type of stability has been precisely investigated for systems of the form (1.1)-(1.3) and some necessary and sufficient conditions of strong stability and instability have been proved. The proofs are based on such a powerful tool as the existence of a Riesz basis of $\mathcal{A}$-invariant finite-dimensional subspaces of the state space and on further application of the results on strong stability in Banach spaces, which originated in [33] and was later developed in $[1,18,31,32]$ and many others (see e.g. [35] for a review). A recent development was described by one of the authors [30]. In this work, the concept of a maximal asymptotic was introduced and conditions of the lack of maximal asymptotic were given, which is a generalization of the strong stability condition. Another approach is to characterize non-closed subspaces of initial conditions, where the rate of convergence to 0 is polynomial (see $[2,5]$ and our Rem. 5.2).

In the case of neutral type systems with a singular neutral term $\left(\operatorname{det} A_{-1}=0\right.$ and $A_{-1} \neq 0$ ), which we call mixed retarded-neutral type systems, strong stability may also occur. However, the approach given in [27,28] cannot be directly applied to such systems, since the existence of a Riesz basis of $\mathcal{A}$-invariant finite-dimensional subspaces of the whole state space cannot be guaranteed. Moreover, mixed retarded-neutral type systems, in general, cannot be decomposed into systems of pure neutral and pure retarded types. Therefore, the analysis of strong stability for mixed retarded-neutral type systems poses a difficult problem, which requires additional essential ideas.

Other approaches are also hard to use for neutral type systems. In particular, in the frequency-domain approach to the stability of delay systems, neutral type systems are difficult to analyze since they have chains of poles close to the imaginary axis (see some recent results in $[4,22]$ ).

The method presented in this paper is based on a decomposition of the initial infinite dimensional model $\dot{x}=\mathcal{A} x$ into two systems $\dot{x}_{0}=\mathcal{A}_{0} x_{0}$ and $\dot{x}_{1}=\mathcal{A}_{1} x_{1}$ in such a way that the spectra of $\mathcal{A}_{0}, \mathcal{A}_{1}$ satisfy: $\operatorname{Re} \sigma\left(\mathcal{A}_{0}\right) \leq-\varepsilon$ and $-\varepsilon<\operatorname{Re} \sigma\left(\mathcal{A}_{1}\right)<0$ for some $\varepsilon>0$. Generally speaking, the operators $\mathcal{A}_{0}$ and $\mathcal{A}_{1}$ are not the models of delay systems (retarded or neutral), which means, in particular, that the relation between their 
exponential growth and spectrum's location is a priori unknown. We prove the exponential stability of the operator $\mathcal{A}_{0}$ by analyzing the boundedness of its resolvent. This direct analysis requires subtle estimates and the proof is technically complicated. For the analysis of the subsystem $\dot{x}_{1}=\mathcal{A}_{1} x_{1}$, we apply methods of strong stability introduced in $[27,28]$. Finally, the introduced approach allows us to prove for mixed retarded-neutral type systems the results on strong stability formulated in [28] for pure neutral type systems $\left(\operatorname{det} A_{-1} \neq 0\right)$.

Besides, for control systems, the proposed approach can analyze the notion of regular asymptotic stabilizability [29] which is closely related to the strong stability notion. The techniques of regular asymptotic stabilizability were introduced in [29] and the sufficient condition for the system (1.1)-(1.3) to be stabilizable was proved in the case $\operatorname{det} A_{-1} \neq 0$. In the present paper, using the same framework as for stability, we show that these results also hold for mixed retarded-neutral type systems.

The general framework is the theory of $C_{0}$-semigroups of linear bounded operators (see e.g. [35]). In order to specify the main contribution of our paper, let us first give the operator model of the system (1.1)-(1.3). We consider first the system without control $(u \equiv 0)$, i.e. given by the equation

$$
\dot{z}(t)=A_{-1} \dot{z}(t-1)+\int_{-1}^{0} A_{2}(\theta) \frac{\mathrm{d}}{\mathrm{d} \theta} z(t+\theta) \mathrm{d} \theta+\int_{-1}^{0} A_{3}(\theta) z(t+\theta) \mathrm{d} \theta, \quad t \geq 0 .
$$

We use the model introduced by Burns et al. [7] in a Hilbert state space. The state space is $M_{2}\left(-1,0 ; \mathbb{C}^{n}\right) \stackrel{\text { def }}{=} \mathbb{C}^{n} \times$ $L_{2}\left(-1,0 ; \mathbb{C}^{n}\right)$, abbreviated $M_{2}$. The operator model is given by the equation

$$
\dot{x}=\mathcal{A} x, \quad x(t)=\left(\begin{array}{c}
y(t) \\
z_{t}(\cdot)
\end{array}\right) \in M_{2},
$$

where the state operator is defined by

$$
\mathcal{A} x(t)=\mathcal{A}\left(\begin{array}{c}
y(t) \\
z_{t}(\cdot)
\end{array}\right)=\left(\begin{array}{c}
\int_{-1}^{0} A_{2}(\theta) \frac{\mathrm{d}}{\mathrm{d} \theta} z_{t}(\theta) \mathrm{d} \theta+\int_{-1}^{0} A_{3}(\theta) z_{t}(\theta) \mathrm{d} \theta \\
\mathrm{d} z_{t}(\theta) / \mathrm{d} \theta
\end{array}\right),
$$

with the domain

$$
\mathcal{D}(\mathcal{A})=\left\{(y, z(\cdot))^{T}: z \in H^{1}\left(-1,0 ; \mathbb{C}^{n}\right), y=z(0)-A_{-1} z(-1)\right\} \subset M_{2}
$$

The operator $\mathcal{A}$ is the infinitesimal generator of a $C_{0}$-semigroup. The solutions of (1.4) and (1.5) are related as $z_{t}(\theta)=z(t+\theta), \theta \in[-1,0]$.

For the controlled system (1.1)-(1.3), i.e. for the equation

$$
\dot{z}(t)=A_{-1} \dot{z}(t-1)+\int_{-1}^{0} A_{2}(\theta) \frac{\mathrm{d}}{\mathrm{d} \theta} z(t+\theta) \mathrm{d} \theta+\int_{-1}^{0} A_{3}(\theta) z(t+\theta) \mathrm{d} \theta+B u,
$$

the corresponding operator model is

$$
\dot{x}=\mathcal{A} x+\mathcal{B} u,
$$

where the operator $\mathcal{B}: \mathbb{C}^{p} \rightarrow M_{2}$ is defined by the $n \times p$-matrix $B$ as follows: $\mathcal{B} u \stackrel{\text { def }}{=}(B u, 0)^{T}$.

The operator $\mathcal{A}$ given by (1.6) possesses only a discrete spectrum $\sigma(\mathcal{A})$ and, moreover, the growth of the semigroup $\left\{\mathrm{e}^{t \mathcal{A}}\right\}_{t \geq 0}$ is determined by the spectrum's location. Namely, denoting by $\omega_{s}=\sup \{\operatorname{Re} \lambda: \lambda \in \sigma(\mathcal{A})\}$ and by $\omega_{0}=\inf \left\{\omega:\left\|\mathrm{e}^{\mathcal{A} t} x\right\| \leq M \mathrm{e}^{\omega t}\|x\|\right\}$, we have the relation $\omega_{0}=\omega_{s}$ (see e.g. [10], Thm. 4.3).

For the stability problem, the last fact implies that the semigroup $\left\{\mathrm{e}^{t \mathcal{A}}\right\}_{t \geq 0}$ is exponentially stable if and only if the spectrum of $\mathcal{A}$ satisfies $\omega_{s}<0$. However, this type of stability is not the only possible one for systems of the form (1.4) (e.g. the same situation can also happen for some hyperbolic partial differential equations). Namely, if $\omega_{s}=0$ (and $A_{-1} \neq 0$ ), then there may be a sequence of eigenvalues with real parts approaching zero 
and imaginary parts tending to infinity. In this critical case, exponential stability is not possible: $\left\|\mathrm{e}^{t \mathcal{A}}\right\| \not 0$ when $t \rightarrow \infty$, but asymptotic non-exponential stability may occur: $\lim _{t \rightarrow+\infty} \mathrm{e}^{t \mathcal{A}} x=0$ for all $x \in M_{2}$.

For system (1.5), satisfying the assumption $\operatorname{det} A_{-1} \neq 0$, the problem of strong stability has been analyzed in $[27,28]$. The main result obtained may be formulated as follows.

Theorem 1.1 ([28], Rabah et al.). Consider the system (1.5) such that $\operatorname{det} A_{-1} \neq 0$. Let us put $\sigma_{1}=\sigma\left(A_{-1}\right) \cap$ $\{\mu:|\mu|=1\}$. Assume that $\sigma(\mathcal{A}) \subset\{\lambda: \operatorname{Re} \lambda<0\}$ (necessary condition). Then one (and only one) of the following three statements is true:

(i) $\sigma_{1}$ consists of simple eigenvalues only, i.e. a one-dimensional eigenspace corresponds to each eigenvalue and there are no root vectors. Then system (1.5) is asymptotically stable.

(ii) The matrix $A_{-1}$ has a Jordan block, corresponding to an eigenvalue $\mu \in \sigma_{1}$. Then (1.5) is unstable.

(iii) There are no Jordan blocks, corresponding to eigenvalues from $\sigma_{1}$, but there is an eigenvalue $\mu \in \sigma_{1}$ whose eigenspace is at least two-dimensional. In this case, system (1.5) can be either stable or unstable. Moreover, there are two systems with the same spectrum, such that one of them is stable while the other is unstable.

Let us discuss the importance of the assumption $\operatorname{det} A_{-1} \neq 0$. The proof of Theorem 1.1 given in [28] is based on the following facts. Firstly, if $\operatorname{det} A_{-1} \neq 0$, then the spectrum of $\mathcal{A}$ is located in a vertical strip $d_{1} \leq \operatorname{Re} \sigma(\mathcal{A}) \leq d_{2}$. Namely, in $[27,28]$ it was shown that $\sigma(\mathcal{A})=\left\{\ln \left|\mu_{m}\right|+\mathrm{i}\left(\arg \mu_{m}+2 \pi k\right)+O(1 / k): \mu_{m} \in\right.$ $\left.\sigma\left(A_{-1}\right), k \in \mathbb{Z}\right\}$. From the latter, the necessary condition for the system to be asymptotically stable also follows: $\sigma\left(A_{-1}\right) \subset\{\mu:|\mu| \leq 1\}$.

Secondly, such a location of the spectrum proves the existence of a Riesz basis of generalized eigenvectors for the operator $\mathcal{A}=\widetilde{\mathcal{A}}$ corresponding to the case $A_{2}(\theta) \equiv A_{3}(\theta) \equiv 0$. For a general operator $\mathcal{A}$, the generalized eigenvectors may not constitute a basis of the state space (see an example in [27] and some general conditions in [36]). However, in $[27,28]$ the existence of a Riesz basis of $\mathcal{A}$-invariant finite-dimensional subspaces of the space $M_{2}$ was proved (see also [37]). Such a basis is a powerful tool that has been applied for the analysis of strong stability.

If we allow the matrix $A_{-1}$ to be singular, then the location of the spectrum described above does not hold anymore. Generally speaking, in this case, for any $\alpha \in \mathbb{R}$ there is an infinite number of eigenvalues which are situated on the left of the vertical line $\operatorname{Re} \lambda=\alpha$. Thus, the existence of a Riesz basis of $\mathcal{A}$-invariant finitedimensional subspaces for the whole space $M_{2}$ cannot be guaranteed. As a consequence, the proof of the item (i) of Theorem 1.1 given in [28], which is essentially based on the Riesz basis technique, is no longer satisfactory and the stability needs to be analyzed another way.

However, it can be asserted that nonzero $\mu_{m} \in \sigma\left(A_{-1}\right)$ define the spectral set $\left\{\ln \left|\mu_{m}\right|+\mathrm{i}\left(\arg \mu_{m}+2 \pi k\right)+\right.$ $\left.O(1 / k): \mu_{m} \in \sigma\left(A_{-1}\right), \mu_{m} \neq 0, k \in \mathbb{Z}\right\} \subset \sigma(\mathcal{A})$ which belongs to a vertical strip. In particular, this can be asserted for $\mu_{m} \in \sigma_{1}$. The fact that Theorem 1.1 is formulated in terms of $\sigma_{1}$ and the last remark gave us the idea to decompose the initial system (1.5) into two systems

$$
\dot{x}=\mathcal{A} x \Leftrightarrow\left\{\begin{array}{c}
\dot{x_{0}}=\mathcal{A}_{0} x_{0} \\
\dot{x_{1}}=\mathcal{A}_{1} x_{1}
\end{array}\right.
$$

in such a way that $\sigma\left(\mathcal{A}_{0}\right)=\sigma(\mathcal{A}) \cap\{\lambda:-\infty<\operatorname{Re} \lambda \leq-\varepsilon\}$ and $\sigma\left(\mathcal{A}_{1}\right)=\sigma(\mathcal{A}) \cap\left\{\lambda:-\varepsilon<\operatorname{Re} \lambda \leq \omega_{s}=0\right\}$, for some $\varepsilon>0$.

To obtain the representation (1.9), we construct a special spectral decomposition of the state space: $M_{2}=$ $M_{2}^{0} \oplus M_{2}^{1}$, where $M_{2}^{0}, M_{2}^{1}$ are $\mathcal{A}$-invariant subspaces. We define $\mathcal{A}_{0}=\left.\mathcal{A}\right|_{M_{2}^{0}}$ and $\mathcal{A}_{1}=\left.\mathcal{A}\right|_{M_{2}^{1}}$.

The spectrum of the system $\dot{x_{1}}=\mathcal{A}_{1} x_{1}$ is such that the corresponding eigenvectors form a Riesz basis of the subspace $M_{2}^{1}$. The strong stability of the semigroup $\left\{\left.\mathrm{e}^{t \mathcal{A}}\right|_{M_{2}^{1}}\right\}_{t \geq 0}$ is proved using the methods of [28].

The semigroup $\left\{\left.\mathrm{e}^{t \mathcal{A}}\right|_{M_{2}^{0}}\right\}_{t \geq 0}$ is exponentially stable. We prove this fact using the equivalent condition consisting of the uniform boundedness of the resolvent $\left.R(\lambda, \mathcal{A})\right|_{M_{2}^{0}}$ on the set $\{\lambda: \operatorname{Re} \lambda \geq 0\}$. Thus, we prove that the initial system $\dot{x}=\mathcal{A} x$ is asymptotically stable. The mentioned scheme requires complicated technique. 
To complete the stability analysis, we revisit the example showing the item (iii) with a simpler formulation than in [28], where it was given using the Riesz basis technique. The analysis of the spectrum carried out in our example is essentially based on the deep results on transcendental equations obtained by Pontryagin [23]. We notice also that the proof of the item (ii) given in [28] does not involve the Riesz basis technique and that it is the same for the case $\operatorname{det} A_{-1}=0$.

The techniques of direct spectral decompositions and resolvent boundedness presented above allow us to extend the results to the stabilizability problem given in [29] for the case of a singular matrix $A_{-1}$.

The general problem of stabilizability of control systems is to find a feedback $u=\mathcal{F} x$ such that the closed-loop system $\dot{x}=(\mathcal{A}+\mathcal{B F}) x$ is asymptotically stable in some sense. For the system (1.7), the result of exponential stabilizability may be derived from those obtained for some particular cases (see e.g. [9,11,20,21]). The required feedback for our system is of the form

$$
F(z(t+\cdot))=F_{-1} \dot{z}(t-1)+\int_{-1}^{0} F_{2}(\theta) \dot{z}(t+\theta) \mathrm{d} \theta+\int_{-1}^{0} F_{3}(\theta) z(t+\theta) \mathrm{d} \theta .
$$

Our purpose is to obtain, as in [29], the condition of asymptotic non-exponential stabilizability of the system (1.7) with the regular feedback

$$
F(z(t+\cdot))=\int_{-1}^{0} F_{2}(\theta) \dot{z}(t+\theta) \mathrm{d} \theta+\int_{-1}^{0} F_{3}(\theta) z(t+\theta) \mathrm{d} \theta,
$$

where $F_{2}(\cdot), F_{3}(\cdot) \in L_{2}\left(-1,0 ; \mathbb{C}^{n \times p}\right)$. The motivation is that this kind of feedback is relatively bounded with respect to the state operator $\mathcal{A}$ and does not change the domain of $\mathcal{A}: \mathcal{D}(\mathcal{A})=\mathcal{D}(\mathcal{A}+\mathcal{B F})$. The natural necessary condition of regular stabilizability is $\sigma\left(A_{-1}\right) \subset\{\mu:|\mu| \leq 1\}$ because $A_{-1}$ is not modified by the feedback. Under the same restrictive condition $\operatorname{det} A_{-1} \neq 0$ in [29], the following result on stabilizability was obtained.

Theorem 1.2 ([29], Rabah et al.). Let the system (1.7) verify the following assumptions:

(1) All the eigenvalues of the matrix $A_{-1}$ satisfy $|\mu| \leq 1$.

(2) All the eigenvalues $\mu \in \sigma_{1}=\sigma\left(A_{-1}\right) \cap\{\mu:|\mu|=1\}$ are simple.

Then the system is regularly asymptotically stabilizable if

(3) $\operatorname{rank}(\Delta(\lambda), B)=n$ for all $\lambda: \operatorname{Re} \lambda \geq 0$,

(4) $\operatorname{rank}\left(\mu I-A_{-1}, B\right)=n$ for all $\mu \in \sigma_{1}$.

The proof of this theorem given in [29] uses the existence of a Riesz basis of finite-dimensional eigenspaces of the whole state space $M_{2}$ and, thus, it requires the assumption $\operatorname{det} A_{-1} \neq 0$. To avoid this assumption, we construct and prove another spectral decomposition which takes into account the unstable part of the system. By means of this decomposition, we separate a subsystem which is generated by the part of the spectrum corresponding to the zero eigenvalues, i.e. the singularities of the matrix $A_{-1}$. Proving the resolvent boundedness, we show the exponential stability of this subsystem. The main "critical" part of the system is in $\mathcal{A}$-invariant subspaces, where we apply the same methods as were given in [29], namely, the theorem on infinite pole assignment, introduced there, and a classical pole assignment result in finite dimensional spaces.

The paper is organized as follows. In Section 2, we recall the results on the spectrum, eigenvectors and the resolvent of the operator $\mathcal{A}$ obtained in [28,29]. Besides, we prove some properties of eigenvectors. In Section 3 , we construct and prove two direct spectral decompositions of the state space. One of them is used to prove the main result on stability and the other for the proof of the result on stabilizability. Section 4 is devoted to the proof of the uniform boundedness of the restriction of the resolvent on some invariant subspaces. Finally, in Sections 5 and 6 , we give the formulation and the proof of our main results on stability and stabilizability. In addition, in Section 5 we give an explicit example of two systems having the same spectrum in the open left half-plane but one of these systems is asymptotically stable while the other is unstable. 


\section{Preliminaries}

In this section, we recall several results on the location of the spectrum of the operator $\mathcal{A}$, on the explicit form of its resolvent and on the form of the eigenvectors of $\mathcal{A}$ and $\mathcal{A}^{*}$. We prove some properties of the eigenvectors of $\mathcal{A}$ and $\mathcal{A}^{*}$.

\subsection{The resolvent and the spectrum}

The results given in this subsection have been presented and proved in [27-29]. Some formulations of the propositions are adapted for the case $\operatorname{det} A_{-1}=0$.

Proposition 2.1 ([28], Prop. 1). The resolvent of the operator $\mathcal{A}$ has the following form:

$$
R(\lambda, \mathcal{A})\left(\begin{array}{c}
z \\
\xi(\cdot)
\end{array}\right) \equiv\left(\begin{array}{c}
\mathrm{e}^{-\lambda} A_{-1} \int_{-1}^{0} \mathrm{e}^{-\lambda s} \xi(s) \mathrm{d} s+\left(I-\mathrm{e}^{-\lambda} A_{-1}\right) \Delta^{-1}(\lambda) D(z, \xi, \lambda) \\
\int_{0}^{\theta} \mathrm{e}^{\lambda(\theta-s)} \xi(s) \mathrm{d} s+\mathrm{e}^{\lambda \theta} \Delta^{-1}(\lambda) D(z, \xi, \lambda)
\end{array}\right),
$$

where $z \in \mathbb{C}^{n}, \xi(\cdot) \in L_{2}\left(-1,0 ; \mathbb{C}^{n}\right) ; \Delta(\lambda)$ is the matrix function defined by

$$
\Delta(\lambda)=\Delta_{\mathcal{A}}(\lambda)=-\lambda I+\lambda \mathrm{e}^{-\lambda} A_{-1}+\lambda \int_{-1}^{0} \mathrm{e}^{\lambda s} A_{2}(s) \mathrm{d} s+\int_{-1}^{0} \mathrm{e}^{\lambda s} A_{3}(s) \mathrm{d} s,
$$

and $D(z, \xi, \lambda)$ is the following vector-function with values in $\mathbb{C}^{n}$ :

$$
\begin{aligned}
D(z, \xi, \lambda)= & z+\lambda \mathrm{e}^{-\lambda} A_{-1} \int_{-1}^{0} \mathrm{e}^{-\lambda \theta} \xi(\theta) \mathrm{d} \theta-\int_{-1}^{0} A_{2}(\theta) \xi(\theta) \mathrm{d} \theta \\
& -\int_{-1}^{0} \mathrm{e}^{\lambda \theta}\left[\lambda A_{2}(\theta)+A_{3}(\theta)\right]\left[\int_{0}^{\theta} \mathrm{e}^{-\lambda s} \xi(s) \mathrm{d} s\right] \mathrm{d} \theta
\end{aligned}
$$

From (2.1), one may see that the resolvent does not exist in the points of singularity of the matrix $\Delta(\lambda)$, i.e. the equation $\operatorname{det} \Delta(\lambda)=0$ defines the eigenvalues of the operator $\mathcal{A}$. Now let us characterize the spectrum of $\mathcal{A}$ more precisely.

We denote by $\mu_{1}, \ldots, \mu_{\ell}$ the set of distinct eigenvalues of the matrix $A_{-1}$ and by $p_{1}, \ldots, p_{\ell}$ their multiplicities. We recall the notation $\sigma_{1}=\sigma\left(A_{-1}\right) \cap\{\mu:|\mu|=1\}$ and assume that $\sigma_{1}=\left\{\mu_{1}, \ldots, \mu_{\ell_{1}}\right\}, \ell_{1} \leq \ell$. We notice that one of the eigenvalues $\mu_{\ell_{1}+1}, \ldots, \mu_{\ell}$ may be zero.

Further, when studying stability and stabilizability problems, we consider mainly the situations when the eigenvalues from $\sigma_{1}$ are simple. This gives us a motivation to assume below (if the opposite is not mentioned) that $p_{1}=\ldots=p_{\ell_{1}}=1$. Besides, without loss of generality, we assume that the matrix $A_{-1}$ is in the following Jordan form:

$$
A_{-1}=\left(\begin{array}{cccccc}
\mu_{1} & \ldots & 0 & 0 & \ldots & 0 \\
\vdots & \ddots & \vdots & \vdots & \ddots & \vdots \\
0 & \ldots & \mu_{\ell_{1}} & 0 & \ldots & 0 \\
0 & \ldots & 0 & J_{\ell_{1}+1} & \ldots & 0 \\
\vdots & \ddots & \vdots & \vdots & \ddots & \vdots \\
0 & \ldots & 0 & 0 & \ldots & J_{\ell}
\end{array}\right)
$$

where $J_{\ell_{1}+1}, \ldots, J_{\ell}$ are Jordan blocks corresponding to the eigenvalues $\mu_{\ell_{1}+1}, \ldots, \mu_{\ell}$.

Let us denote by $\widetilde{\mathcal{A}}$ the state operator in the case when $A_{2}(\theta) \equiv A_{3}(\theta) \equiv 0$. It is not difficult to see that the spectrum of $\widetilde{\mathcal{A}}$ has the following structure

$$
\sigma(\widetilde{\mathcal{A}})=\left\{\widetilde{\lambda}_{m}^{k}=\ln \left|\mu_{m}\right|+\mathrm{i}\left(\arg \mu_{m}+2 \pi k\right): m=1, \ldots, \ell, \mu_{m} \neq 0, k \in \mathbb{Z}\right\} \cup\{0\} .
$$

We denote by $L_{m}^{k}\left(r^{(k)}\right)$ the circles centered at $\widetilde{\lambda}_{m}^{k}$ with radii $r^{(k)}$. 
Proposition 2.2. Let $\sigma_{1}=\left\{\mu_{1}, \ldots, \mu_{\ell_{1}}\right\}$ consist of simple eigenvalues only. There exists $N_{1} \in \mathbb{N}$ such that the total multiplicity of the roots of the equation $\operatorname{det} \Delta(\lambda)=0$, contained in the circles $L_{m}^{k}\left(r^{(k)}\right)$, equals $p_{m}=1$ for all $m=1, \ldots, \ell_{1}$ and $k:|k| \geq N_{1}$, and the radii $r^{(k)}$ satisfy the relation $\sum_{k \in \mathbb{Z}}\left(r^{(k)}\right)^{2}<\infty$.

This proposition is a particular case of [29], Theorem 4, which was formulated and proved there under the assumption $\operatorname{det} A_{-1} \neq 0$. The proof for the case $\operatorname{det} A_{-1}=0$ remains the same. A similar result was obtained for systems given by transfer functions in [4,22].

Notation 2.3. We denote the eigenvalues of $\mathcal{A}$ mentioned in Proposition 2.2 by $\lambda_{m}^{k}, m=1, \ldots, \ell_{1},|k| \geq N_{1}$.

Remark 2.4. Proposition 2.2 is formulated for $m=1, \ldots, \ell_{1}$, however, it also holds for all those indices $m=1, \ldots, \ell$ which correspond to nonzero eigenvalues $\mu_{m} \in \sigma\left(A_{-1}\right)$.

Remark 2.5. In the case $\operatorname{det} A_{-1} \neq 0$, the spectrum of $\mathcal{A}$ belongs to a vertical strip which is bounded from the left and from the right. However, in the case $\operatorname{det} A_{-1}=0$, in addition to the eigenvalues mentioned in Proposition 2.2, the operator $\mathcal{A}$ may also possess an infinite sequence of eigenvalues with real parts tending to $-\infty$

Similar results hold for the operator $\mathcal{A}^{*}$. The spectra of $\mathcal{A}$ and $\mathcal{A}^{*}$ are related as $\sigma\left(\mathcal{A}^{*}\right)=\overline{\sigma(\mathcal{A})}$. Eigenvalues of $\mathcal{A}^{*}$ are the roots of the equation $\operatorname{det} \Delta^{*}(\lambda)=0$, where

$$
\Delta^{*}(\lambda)=\Delta_{\mathcal{A}^{*}}(\lambda)=-\lambda I+\lambda \mathrm{e}^{-\lambda} A_{-1}^{*}+\lambda \int_{-1}^{0} \mathrm{e}^{\lambda s} A_{2}^{*}(s) \mathrm{d} s+\int_{-1}^{0} \mathrm{e}^{\lambda s} A_{3}^{*}(s) \mathrm{d} s,
$$

and the relation $(\Delta(\lambda))^{*}=\Delta^{*}(\bar{\lambda})$ holds. The eigenvalues $\overline{\lambda_{m}^{k}}, m=1, \ldots, \ell_{1},|k| \geq N_{1}$ may be described as in Proposition 2.2.

\subsection{Eigenvectors of $\mathcal{A}$ and $\mathcal{A}^{*}$}

First, we give the explicit form of eigenvectors given in [28,29].

Proposition 2.6 ([28], Thm. 2, [29], Thm. 7). The eigenvectors $\varphi$ and $\psi$ of $\mathcal{A}$ and $\mathcal{A}^{*}$, respectively, are given by:

$$
\begin{gathered}
\varphi=\varphi(\lambda)=\left(\begin{array}{c}
\left(I-\mathrm{e}^{-\lambda} A_{-1}\right) x \\
\mathrm{e}^{\lambda \theta} x
\end{array}\right), \\
\psi=\psi(\bar{\lambda})=\left(\left[\bar{\lambda} \mathrm{e}^{-\bar{\lambda} \theta}-A_{2}^{*}(\theta)+\mathrm{e}^{-\bar{\lambda} \theta} \int_{0}^{\theta} \mathrm{e}^{\bar{\lambda} s}\left(A_{3}^{*}(s)+\bar{\lambda} A_{2}^{*}(s)\right) \mathrm{d} s\right] y\right),
\end{gathered}
$$

where $x=x(\lambda) \in \operatorname{Ker} \Delta(\lambda), x \neq 0$ and $y=y(\bar{\lambda}) \in \operatorname{Ker} \Delta^{*}(\bar{\lambda}), y \neq 0$.

Below we give several properties of the sets of eigenvectors and we begin with the calculation of the scalar product between the eigenvectors of $\mathcal{A}$ and $\mathcal{A}^{*}$.

Lemma 2.7. Let $\lambda_{0}, \lambda_{1} \in \sigma(\mathcal{A})$ and $\varphi=\varphi\left(\lambda_{0}\right), \psi=\psi\left(\overline{\lambda_{1}}\right)$ be the corresponding eigenvectors: $\left(\mathcal{A}-\lambda_{0} I\right) \varphi=0$, $\left(\mathcal{A}^{*}-\overline{\lambda_{1}} I\right) \psi=0$. Then the scalar product $\langle\varphi, \psi\rangle_{M_{2}}$ is given by:

$$
\langle\varphi, \psi\rangle_{M_{2}}=\left\{\begin{array}{cc}
0, & \text { for } \lambda_{0} \neq \lambda_{1} \\
-\left\langle\Delta^{\prime}\left(\lambda_{0}\right) x, y\right\rangle_{\mathbb{C}^{n}}, & \text { for } \lambda_{0}=\lambda_{1}
\end{array}\right.
$$

where $\Delta^{\prime}(\lambda)=\frac{\mathrm{d}}{\mathrm{d} \lambda} \Delta(\lambda)$ and $x=x\left(\lambda_{0}\right), y=y\left(\overline{\lambda_{1}}\right)$ are defined by (2.5) and (2.6). 
Proof. Since $\varphi\left(\lambda_{0}\right)$ and $\psi\left(\overline{\lambda_{1}}\right)$ are eigenvectors of adjoint operators, then $\langle\varphi, \psi\rangle_{M_{2}}=0$ when $\lambda_{0} \neq \lambda_{1}$. Let us consider the case $\lambda_{0}=\lambda_{1}$ and compute directly the scalar product $\langle\varphi, \psi\rangle_{M_{2}}$ using the representations (2.5)-(2.6):

$$
\begin{aligned}
\langle\varphi, \psi\rangle_{M_{2}}= & \left\langle\left(I-\mathrm{e}^{-\lambda_{0}} A_{-1}\right) x, y\right\rangle_{\mathbb{C}^{n}}+\int_{-1}^{0}\left\langle\mathrm{e}^{\lambda_{0} \theta} x, \overline{\lambda_{0}} \mathrm{e}^{-\overline{\lambda_{0}} \theta} y\right\rangle_{\mathbb{C}^{n}} \mathrm{~d} \theta-\int_{-1}^{0}\left\langle\mathrm{e}^{\lambda_{0} \theta} x, A_{2}^{*}(\theta) y\right\rangle_{\mathbb{C}^{n}} \mathrm{~d} \theta \\
& +\int_{-1}^{0}\left\langle\mathrm{e}^{\lambda_{0} \theta} x, \mathrm{e}^{-\overline{\lambda_{0}} \theta} \int_{0}^{\theta} \mathrm{e}^{\overline{\lambda_{0}} s}\left(A_{3}^{*}(s)+\overline{\lambda_{0}} A_{2}^{*}(s)\right) \mathrm{d} s \cdot y\right\rangle_{\mathbb{C}^{n}} \mathrm{~d} \theta \\
= & \left\langle\left(I-\mathrm{e}^{-\lambda_{0}} A_{-1}\right) x, y\right\rangle+\left\langle\int_{-1}^{0} \lambda_{0} \mathrm{~d} \theta \cdot x, y\right\rangle_{\mathbb{C}^{n}}-\left\langle\int_{-1}^{0} \mathrm{e}^{\lambda_{0} \theta} A_{2}(\theta) \mathrm{d} \theta \cdot x, y\right\rangle_{\mathbb{C}^{n}} \\
& +\left\langle\int_{-1}^{0} \int_{0}^{\theta} \mathrm{e}^{\lambda_{0} s}\left(A_{3}(s)+\lambda_{0} A_{2}(s)\right) \mathrm{d} s \mathrm{~d} \theta \cdot x, y\right\rangle_{\mathbb{C}^{n}} \\
= & \left\langle\Gamma\left(\lambda_{0}\right) x, y\right\rangle_{\mathbb{C}^{n},}
\end{aligned}
$$

where

$$
\Gamma\left(\lambda_{0}\right)=I-\mathrm{e}^{-\lambda_{0}} A_{-1}+\lambda_{0} I-\int_{-1}^{0} \mathrm{e}^{\lambda_{0} \theta} A_{2}(\theta) \mathrm{d} \theta+\int_{-1}^{0} \int_{0}^{\theta} \mathrm{e}^{\lambda_{0} s}\left(A_{3}(s)+\lambda_{0} A_{2}(s)\right) \mathrm{d} s \mathrm{~d} \theta .
$$

The last term of $\Gamma\left(\lambda_{0}\right)$, which is the integral over the domain $-1 \leq \theta \leq s \leq 0$, we rewrite using the identity $\int_{-1}^{0} \int_{0}^{\theta} G(s, \theta) \mathrm{d} s \mathrm{~d} \theta=-\int_{-1}^{0} \int_{-1}^{s} G(s, \theta) \mathrm{d} \theta \mathrm{d} s$. Thus, we obtain:

$$
\begin{aligned}
\Gamma\left(\lambda_{0}\right)= & I-\mathrm{e}^{-\lambda_{0}} A_{-1}+\lambda_{0} I-\int_{-1}^{0} \mathrm{e}^{\lambda_{0} \theta} A_{2}(\theta) \mathrm{d} \theta-\int_{-1}^{0} \mathrm{e}^{\lambda_{0} s}\left(A_{3}(s)+\lambda_{0} A_{2}(s)\right) \int_{-1}^{s} \mathrm{~d} \theta \mathrm{d} s \\
= & \left(I-\mathrm{e}^{-\lambda_{0}} A_{-1}-\int_{-1}^{0} \mathrm{e}^{\lambda_{0} s}\left(s A_{3}(s)+s \lambda_{0} A_{2}(s)+A_{2}(s)\right) \mathrm{d} s\right) \\
& +\left(\lambda_{0} I-\int_{-1}^{0} \mathrm{e}^{\lambda_{0} s}\left(A_{3}(s)+\lambda_{0} A_{2}(s)\right) \mathrm{d} s\right) \\
= & -\Delta^{\prime}\left(\lambda_{0}\right)-\Delta\left(\lambda_{0}\right) .
\end{aligned}
$$

Taking into account the relation $x \in \operatorname{Ker} \Delta\left(\lambda_{0}\right)$, we conclude that

$$
\langle\varphi, \psi\rangle_{M_{2}}=-\left\langle\Delta^{\prime}\left(\lambda_{0}\right) x, y\right\rangle_{\mathbb{C}^{n}}
$$

The latter completes the proof of the lemma.

For $\varphi\left(\lambda_{m}^{k}\right)$ and $\psi\left(\overline{\lambda_{m}^{k}}\right)$ we will use the notation $\varphi_{m}^{k}$ and $\psi_{m}^{k}$ respectively. Besides, we use $x_{m}^{k}$ and $y_{m}^{k}$ instead of $x\left(\lambda_{m}^{k}\right)$ and $y\left(\overline{\lambda_{m}^{k}}\right)$.

Lemma 2.8. Let $\sigma_{1}=\left\{\mu_{1}, \ldots, \mu_{\ell_{1}}\right\}$ consist of simple eigenvalues only. The eigenvectors $\varphi_{m}^{k}, m=1, \ldots, \ell_{1}$, $k:|k| \geq N_{1}$ constitute a Riesz basis of the closure of their linear span. The same holds for eigenvectors $\psi_{m}^{k}$, $m=1, \ldots, \ell_{1}, k:|k| \geq N_{1}$.

A more general formulation of this proposition was given in [27], Theorems 7, 15 under the assumption $\operatorname{det} A_{-1} \neq 0$. We give a sketch of the proof in our case. 
The families of functions $\left\{\mathrm{e}^{\tilde{\lambda}_{m}^{k} \theta}\right\}_{k \in \mathbb{Z}}$ form an orthogonal basis of the space $L_{2}([-1,0], \mathbb{C})$ for each $m=1, \ldots, \ell_{1}$, where $\widetilde{\lambda}_{m}^{k}=\mathrm{i}\left(\arg \mu_{m}+2 \pi k\right)$ are eigenvalues of the operator $\widetilde{\mathcal{A}}$. Thus, the functions $\left\{\widetilde{\mathrm{e}}^{\tilde{\lambda}_{m}^{k} \theta}\right\}_{|k| \geq N}, N \in \mathbb{N}$ form a basis of the closure of their linear span.

Since we have chosen the matrix $A_{-1}$ in the form (2.4) and due to (2.5), the eigenvectors $\widetilde{\varphi}_{m}^{k}$ of $\widetilde{\mathcal{A}}$ are of the form $\widetilde{\varphi}_{m}^{k}=\left(\begin{array}{c}0 \\ \mathrm{e}^{\tilde{\lambda}_{m}^{k} \theta} e_{m}\end{array}\right), e_{m}=(0, \ldots, 1, \ldots, 0)^{T}$. Therefore, the family $\left\{\widetilde{\varphi}_{m}^{k}: m=1, \ldots, \ell_{1}:|k| \geq N_{1}\right\}$ is a basis of the closure of its linear span.

The eigenvectors $\varphi_{m}^{k}=\left(\begin{array}{c}\left(I-\mathrm{e}^{-\lambda_{m}^{k}} A_{-1}\right) x_{m}^{k} \\ \mathrm{e}^{\lambda_{m}^{k} \theta} x_{m}^{k}\end{array}\right)$ of $\mathcal{A}$ are quadratically close to $\widetilde{\varphi}_{m}^{k}$. To prove this fact, we should argue similarly to Theorem 15 given in [27] (based essentially on perturbation theory [14]). Thus, eigenvectors $\varphi_{m}^{k}, m=1, \ldots, \ell_{1}, k:|k| \geq N_{1}$ constitute a Riesz basis of the closure of their linear span.

From Lemma 2.7 and Lemma 2.8, we conclude the following.

Corollary 2.9. The sequences $\varphi_{m}^{k}$ and $\psi_{m}^{k}, m=1, \ldots, \ell_{1}, k:|k| \geq N_{1}$ are biorthogonal after normalization and $\left\langle\varphi_{m}^{k}, \psi_{m}^{k}\right\rangle_{M_{2}}=-\left\langle\Delta^{\prime}\left(\lambda_{m}^{k}\right) x_{m}^{k}, y_{m}^{k}\right\rangle_{\mathbb{C}^{n}}$.

The following relation will be used essentially in the analysis of the boundedness of the resolvent in Section 4 .

Lemma 2.10. Let $\psi=\psi\left(\overline{\lambda_{0}}\right), \lambda_{0} \in \sigma(\mathcal{A})$ be an eigenvector of the operator $\mathcal{A}^{*}$ and let $g=(z, \xi(\cdot)) \in M_{2}$ be orthogonal to $\psi: g \perp \psi$. Then the following relation holds:

$$
D\left(z, \xi, \lambda_{0}\right) \in \operatorname{Im} \Delta\left(\lambda_{0}\right)
$$

where $D(z, \xi, \lambda)$ is defined by $(2.3)$.

Proof. We show the relation $D\left(z, \xi, \lambda_{0}\right) \perp \operatorname{Ker} \Delta^{*}\left(\overline{\lambda_{0}}\right)$ which is equivalent to (2.8). The eigenvector $\psi$ is of the form (2.6):

$$
\psi=\left(\left[\overline{\lambda_{0}} \mathrm{e}^{-\overline{\lambda_{0}} \theta}-A_{2}^{*}(\theta)+\mathrm{e}^{-\overline{\lambda_{0}} \theta} \int_{0}^{\theta} \mathrm{e}^{\overline{\lambda_{0}} s} A_{3}^{*}(s) \mathrm{d} s+\overline{\lambda_{0}} \mathrm{e}^{-\overline{\lambda_{0}} \theta} \int_{0}^{\theta} \mathrm{e}^{\overline{\lambda_{0}} s} A_{2}^{*}(s) \mathrm{d} s\right] y\right),
$$

where $y=y\left(\overline{\lambda_{0}}\right) \in \operatorname{Ker} \Delta^{*}\left(\overline{\lambda_{0}}\right)$. For any $g=(z, \xi(\cdot))$, which is orthogonal to $\psi$, we obtain:

$$
\begin{aligned}
0=\langle g, \psi\rangle_{M_{2}}= & \langle z, y\rangle_{\mathbb{C}^{n}}+\int_{-1}^{0}\left\langle\xi(\theta), \overline{\lambda_{0}} \mathrm{e}^{-\overline{\lambda_{0}} \theta} y\right\rangle_{\mathbb{C}^{n}} \mathrm{~d} \theta-\int_{-1}^{0}\left\langle\xi(\theta), A_{2}^{*}(\theta) y\right\rangle_{\mathbb{C}^{n}} \mathrm{~d} \theta \\
& +\int_{-1}^{0}\left\langle\xi(\theta), \mathrm{e}^{-\overline{\lambda_{0}} \theta} \int_{0}^{\theta} \mathrm{e}^{\overline{\mathrm{A}_{0}} s}\left(A_{3}^{*}(s)+\overline{\lambda_{0}} A_{2}^{*}(s)\right) \mathrm{d} s \cdot y\right\rangle_{\mathbb{C}^{n}} \mathrm{~d} \theta \\
= & \langle z, y\rangle_{\mathbb{C}^{n}}+\left\langle\int_{-1}^{0} \lambda_{0} \mathrm{e}^{-\lambda_{0} \theta} \xi(\theta) \mathrm{d} \theta, y\right\rangle_{\mathbb{C}^{n}}-\left\langle\int_{-1}^{0} A_{2}(\theta) \xi(\theta) \mathrm{d} \theta, y\right\rangle_{\mathbb{C}^{n}} \\
& +\left\langle\int_{-1}^{0}\left[\mathrm{e}^{-\lambda_{0} \theta} \int_{0}^{\theta} \mathrm{e}^{\lambda_{0} s}\left(A_{3}(s)+\lambda_{0} A_{2}(s)\right) \mathrm{d} s\right] \xi(\theta) \mathrm{d} \theta, y\right\rangle_{\mathbb{C}^{n}} .
\end{aligned}
$$


Using the identity $\int_{-1}^{0} \int_{0}^{\theta} G(s, \theta) \mathrm{d} s \mathrm{~d} \theta=-\int_{-1}^{0} \int_{-1}^{s} G(s, \theta) \mathrm{d} \theta \mathrm{d} s$ which holds for any function $G(s, \theta)$, we rewrite the last term of (2.9), and, finally, we obtain the relation:

$$
\begin{aligned}
0=\langle g, \psi\rangle_{M_{2}}= & \left\langle z+\int_{-1}^{0} \lambda_{0} \mathrm{e}^{-\lambda_{0} \theta} \xi(\theta) \mathrm{d} \theta+\int_{-1}^{0} A_{2}(\theta) \xi(\theta) \mathrm{d} \theta\right. \\
& \left.-\int_{-1}^{0} \mathrm{e}^{\lambda_{0} s}\left[A_{3}(s)+\lambda_{0} A_{2}(s)\right] \int_{-1}^{s} \mathrm{e}^{-\lambda_{0} \theta} \xi(\theta) \mathrm{d} \theta \mathrm{d} s, y\right\rangle_{\mathbb{C}^{n}} .
\end{aligned}
$$

Since $y \in \operatorname{Ker} \Delta^{*}\left(\overline{\lambda_{0}}\right)$, then for any $x \in \mathbb{C}^{n}$ we have:

$$
0=\left\langle x, \Delta^{*}\left(\overline{\lambda_{0}}\right) y\right\rangle_{\mathbb{C}^{n}}=\left\langle\Delta\left(\lambda_{0}\right) x, y\right\rangle_{\mathbb{C}^{n}} .
$$

Therefore, for any $\theta$ the relation $\left\langle\mathrm{e}^{-\lambda_{0} \theta} \Delta\left(\lambda_{0}\right) \xi(\theta), y\right\rangle_{\mathbb{C}^{n}}=0$ holds, and, integrating it by $\theta$ from -1 to 0 , we obtain:

$$
\begin{aligned}
0=\left\langle\int_{-1}^{0} \mathrm{e}^{-\lambda_{0} \theta} \Delta\left(\lambda_{0}\right) \xi(\theta) \mathrm{d} \theta, y\right\rangle= & \left\langle-\int_{\mathbb{C}^{n}}^{0} \lambda_{0} \mathrm{e}^{-\lambda_{0} \theta} \xi(\theta) \mathrm{d} \theta+\int_{-1}^{0} \lambda_{0} \mathrm{e}^{-\lambda_{0} \theta} A_{-1} \xi(\theta) \mathrm{d} \theta\right. \\
& \left.+\int_{-1}^{0} \mathrm{e}^{\lambda_{0} s}\left[A_{3}(s)+\lambda_{0} A_{2}(s)\right] \mathrm{d} s \int_{-1}^{0} \mathrm{e}^{-\lambda_{0} \theta} \xi(\theta) \mathrm{d} \theta, y\right\rangle_{\mathbb{C}^{n}}
\end{aligned}
$$

Let us sum up the left-hand sides and the right-hand sides of the relations (2.11) and (2.10). In the obtained relation, the term $\int_{-1}^{0} \lambda_{0} \mathrm{e}^{-\lambda_{0} \theta} \xi(\theta) \mathrm{d} \theta$ is cancelled. The last terms of (2.11) and (2.10) are summed up according to the identity $-\int_{-1}^{0} \int_{-1}^{s} G(s, \theta) \mathrm{d} \theta \mathrm{d} s+\int_{-1}^{0} \int_{-1}^{0} G(s, \theta) \mathrm{d} \theta \mathrm{d} s=-\int_{-1}^{0} \int_{0}^{s} G(s, \theta) \mathrm{d} \theta \mathrm{d} s=-\int_{-1}^{0} \int_{0}^{\theta} G(\theta, s) \mathrm{d} s \mathrm{~d} \theta$ which holds true for any function $G(s, \theta)$. Finally, we obtain:

$$
\begin{aligned}
0= & \left\langle z+\lambda_{0} \mathrm{e}^{-\lambda_{0}} A_{-1} \int_{-1}^{0} \mathrm{e}^{-\lambda_{0} \theta} \xi(\theta) \mathrm{d} \theta-\int_{-1}^{0} A_{2}(\theta) \xi(\theta) \mathrm{d} \theta\right. \\
& \left.-\int_{-1}^{0} \mathrm{e}^{\lambda_{0} \theta}\left[A_{3}(\theta)+\lambda_{0} A_{2}(\theta)\right]\left[\int_{0}^{\theta} \mathrm{e}^{-\lambda_{0} s} \xi(s) \mathrm{d} s\right] \mathrm{d} \theta, y\right\rangle_{\mathbb{C}^{n}} \\
\equiv & \left\langle D\left(z, \xi, \lambda_{0}\right), y\right\rangle_{\mathbb{C}^{n}} .
\end{aligned}
$$

Since $y \in \operatorname{Ker} \Delta^{*}\left(\overline{\lambda_{0}}\right)$, we conclude that $D\left(z, \xi, \lambda_{0}\right) \perp \operatorname{Ker} \Delta^{*}\left(\overline{\lambda_{0}}\right)$. This completes the proof of the lemma.

Remark 2.11. We emphasize the fact that $\operatorname{det} \Delta\left(\lambda_{0}\right)=0$ and, therefore, the matrix $\Delta^{-1}\left(\lambda_{0}\right)$ does not exist. However, the proved relation $D\left(z, \xi, \lambda_{0}\right) \in \operatorname{Im} \Delta\left(\lambda_{0}\right)$ means that the inverse image of the vector $D\left(z, \xi, \lambda_{0}\right)$ with respect to the matrix $\Delta\left(\lambda_{0}\right)$ does exist.

\section{SpeCtral DeCompositions of the state SPACE}

We recall that we consider the operator $\mathcal{A}$ in the case when all eigenvalues from $\sigma_{1} \subset \sigma\left(A_{-1}\right)$ are simple. In this section, we construct two spectral decompositions of the state space $M_{2}$. Assuming that $\sigma(\mathcal{A}) \subset\{\lambda$ : $\operatorname{Re} \lambda<0\}$, in the first subsection we construct a decomposition which we use later in Section 5 for the stability analysis. In the second subsection, we assume only $|\mu| \leq 1$ for all $\mu \in \sigma\left(A_{-1}\right)$ (i.e. a part of the spectrum 
of $\mathcal{A}$ may belong to the closed right half-plane) and construct a decomposition needed in Section 6 for the stabilizability analysis. The structures of these decompositions are very similar. In the third subsection, we prove some technical results used in the proofs of the validity of the decompositions.

\subsection{Spectral decomposition for the stability problem}

For the stability analysis, our aim is to divide the system into an exponentially stable part and a strongly asymptotically stable part. To do this, we construct a decomposition of the state space $M_{2}$ into the direct sum of two $\mathcal{A}$-invariant subspaces and prove its validity.

We divide the spectrum of $\mathcal{A}$ into two parts. For some $N \geq N_{1}$, we define

$$
\Lambda_{1}=\Lambda_{1}(N)=\left\{\lambda_{m}^{k} \in \sigma(\mathcal{A}), m=1, \ldots, \ell_{1},|k| \geq N\right\},
$$

and represent the spectrum as follows:

$$
\sigma(\mathcal{A})=\Lambda_{0} \cup \Lambda_{1}
$$

Remark 3.1. The set $\Lambda_{1}$ is determined by $N \in \mathbb{N}$. For any small $\varepsilon>0$, there is a big enough $N$ such that $\Lambda_{1}$ belongs to the vertical strip $\{\lambda:-\varepsilon<\operatorname{Re} \lambda<0\}$.

We introduce two subspaces of $M_{2}$ :

$$
\begin{aligned}
& M_{2}^{1}=M_{2}^{1}(N)=\mathrm{Cl} \operatorname{Lin}\left\{\varphi:(\mathcal{A}-\lambda I) \varphi=0, \lambda \in \Lambda_{1}\right\}, \\
& \widehat{M}_{2}^{1}=\widehat{M}_{2}^{1}(N)=\mathrm{Cl} \operatorname{Lin}\left\{\psi:\left(\mathcal{A}^{*}-\bar{\lambda} I\right) \psi=0, \lambda \in \Lambda_{1}\right\} .
\end{aligned}
$$

Obviously, $M_{2}^{1}$ is $\mathcal{A}$-invariant and $\widehat{M}_{2}^{1}$ is $\mathcal{A}^{*}$-invariant. We introduce $M_{2}^{0}=M_{2}^{0}(N)$ which satisfies

$$
M_{2}=\widehat{M}_{2}^{1} \stackrel{\perp}{\oplus} M_{2}^{0} .
$$

Due to the construction, $M_{2}^{0}$ is an $\mathcal{A}$-invariant subspace.

Remark 3.2. We recall that, due to Lemma 2.8, eigenvectors $\left\{\varphi_{m}^{k}\right\}$ of $\mathcal{A}$, corresponding to $\lambda_{m}^{k} \in \Lambda_{1}$, form a Riesz basis of the closure of their linear span. The same holds for eigenvectors $\left\{\psi_{m}^{k}\right\}$ of $\mathcal{A}^{*}$, corresponding to $\overline{\lambda_{m}^{k}}, \lambda_{m}^{k} \in \Lambda_{1}$.

The main result of this subsection is the following theorem.

Theorem 3.3 (on direct decomposition). Let $\sigma_{1}=\left\{\mu_{1}, \ldots, \mu_{\ell_{1}}\right\}$ consist of simple eigenvalues only. For any $N \geq N_{1}$, the subset $\Lambda_{1}=\Lambda_{1}(N) \subset \sigma(\mathcal{A})$ given by (3.1) and the subspaces $M_{2}^{0}, M_{2}^{1}, \widehat{M}_{2}^{1}$, given by (3.2), (3.3) and (3.4) define the direct decomposition of the space:

$$
M_{2}=M_{2}^{1} \oplus M_{2}^{0}
$$

where the subspaces $M_{2}^{1}, M_{2}^{0}$ are $\mathcal{A}$-invariant.

Proof. To prove (3.5) we show that any element $\xi \in M_{2}$ allows the following representation:

$$
\xi=\xi_{0}+\sum_{m=1}^{\ell_{1}} \sum_{|k| \geq N} c_{m}^{k} \varphi_{m}^{k}, \quad \xi_{0} \in M_{2}^{0}, \varphi_{m}^{k} \in M_{2}^{1}, \quad \sum_{m=1}^{\ell_{1}} \sum_{|k| \geq N}\left|c_{m}^{k}\right|^{2}<\infty,
$$

where, as we have noticed above, the eigenvectors $\left\{\varphi_{m}^{k}:\left(\mathcal{A}-\lambda_{m}^{k} I\right) \varphi_{m}^{k}=0, \lambda_{m}^{k} \in \Lambda_{1}\right\}$ given by (2.5) form a Riesz basis of the closure of their linear span. Besides, the eigenvectors $\left\{\frac{1}{\bar{\lambda}_{m}^{k}} \psi_{m}^{k}:\left(\mathcal{A}^{*}-\overline{\lambda_{m}^{k}} I\right) \psi_{m}^{k}=0, \lambda_{m}^{k} \in \Lambda_{1}\right\}$ 
form a Riesz basis of the closure of their linear span, where eigenvectors $\psi_{m}^{k}$ are given by (2.6). We use the notation $\widehat{\psi}_{m}^{k}=\frac{1}{\bar{\lambda}_{m}^{k}} \psi_{m}^{k}$.

Using the representations (2.5) and (2.6), we choose eigenvectors with $\left\|x_{m}^{k}\right\|_{\mathbb{C}^{n}}=1$ and $\left\|y_{m}^{k}\right\|_{\mathbb{C}^{n}}=1$. Due to Lemma 3.5 given in Subsection 3.3, there is $C>0$ such that $\left\|\varphi_{m}^{k}\right\|_{M^{2}} \leq C$ and $\left\|\widehat{\psi}_{m}^{k}\right\|_{M^{2}} \leq C$ for all $m=1, \ldots, \ell_{1},|k| \geq N$.

Applying the decomposition (3.4) to vectors $\varphi_{m}^{k}$, we obtain

$$
\varphi_{m}^{k}=\gamma_{m}^{k}+\sum_{i=1}^{\ell_{1}} \sum_{|j| \geq N} a_{i}^{j} \widehat{\psi}_{i}^{j}, \quad \gamma_{m}^{k} \in M_{2}^{0} .
$$

Since $\left\langle\varphi_{m}^{k}, \widehat{\psi}_{i}^{j}\right\rangle=0$ for $(m, k) \neq(i, j)$ (Cor. 2.9), the last representation may be rewritten as follows:

$$
\varphi_{m}^{k}=\gamma_{m}^{k}+a_{m}^{k} \widehat{\psi}_{m}^{k}, \quad \gamma_{m}^{k} \in M_{2}^{0}
$$

Moreover, due to (2.7) we have the relation

$$
a_{m}^{k}=\frac{\left\langle\varphi_{m}^{k}, \widehat{\psi}_{m}^{k}\right\rangle_{M_{2}}}{\left\|\widehat{\psi}_{m}^{k}\right\|_{M_{2}}^{2}}=\frac{\frac{1}{\lambda_{m}^{k}}\left\langle\varphi_{m}^{k}, \psi_{m}^{k}\right\rangle_{M_{2}}}{\left\|\widehat{\psi}_{m}^{k}\right\|_{M_{2}}^{2}}=\frac{-\frac{1}{\lambda_{m}^{k}}\left\langle\Delta^{\prime}\left(\lambda_{m}^{k}\right) x_{m}^{k}, y_{m}^{k}\right\rangle_{\mathbb{C}^{n}}}{\left\|\widehat{\psi}_{m}^{k}\right\|_{M_{2}}^{2}} .
$$

From (3.6) and (3.7) it also follows that

$$
\left\|\gamma_{m}^{k}\right\| \leq\left\|\varphi_{m}^{k}\right\|+\left|a_{m}^{k}\right|\left\|\widehat{\psi}_{m}^{k}\right\| \leq C+\sqrt{\left|\frac{1}{\lambda_{m}^{k}}\left\langle\Delta^{\prime}\left(\lambda_{m}^{k}\right) x_{m}^{k}, y_{m}^{k}\right\rangle\right|}
$$

Using the decomposition (3.4) and the relation (3.6), we represent each vector $\xi \in M_{2}$ as follows:

$$
\begin{aligned}
\xi & =\widehat{\xi}_{0}+\sum_{m=1}^{\ell_{1}} \sum_{|k| \geq N} b_{m}^{k} \widehat{\psi}_{m}^{k}=\widehat{\xi}_{0}-\sum_{m=1}^{\ell_{1}} \sum_{|k| \geq N} \frac{b_{m}^{k}}{a_{m}^{k}} \gamma_{m}^{k}+\sum_{m=1}^{\ell_{1}} \sum_{|k| \geq N} \frac{b_{m}^{k}}{a_{m}^{k}} \varphi_{m}^{k} \\
& =\xi_{0}+\sum_{m=1}^{\ell_{1}} \sum_{|k| \geq N} c_{m}^{k} \varphi_{m}^{k}
\end{aligned}
$$

where $\widehat{\xi}_{0} \in M_{2}^{0}, \sum_{m=1}^{\ell_{1}} \sum_{|k| \geq N}\left|b_{m}^{k}\right|^{2}<\infty, \xi_{0}=\widehat{\xi}_{0}-\sum_{m=1}^{\ell_{1}} \sum_{|k| \geq N} \frac{b_{m}^{k}}{a_{m}^{k}} \gamma_{m}^{k} \in M_{2}^{0}, c_{m}^{k}=\frac{b_{m}^{k}}{a_{m}^{k}}$.

To prove the validity of the decomposition (3.9), it is enough to show that $\left|\frac{1}{a_{m}^{k}}\right| \leq C_{1}$ and $\left\|\gamma_{m}^{k}\right\| \leq C_{2}$ for some $0<C_{1}, C_{2}<+\infty$. Taking into account (3.7) and (3.8), the latter provides the estimate

$$
0<C_{1} \leq\left|\frac{1}{\lambda_{m}^{k}}\left\langle\Delta^{\prime}\left(\lambda_{m}^{k}\right) x_{m}^{k}, y_{m}^{k}\right\rangle\right| \leq C_{2}, \quad \lambda_{m}^{k} \in \Lambda_{1} .
$$

This estimate is proved by Lemma 3.11 given in Subsection 3.3 .

Thus, the representation (3.9) holds for any $\xi \in M_{2}$. The proof of the theorem is now complete.

\subsection{Spectral decomposition for the stabilizability problem}

We recall that for the stabilizability problem we assume only that $|\mu| \leq 1$ for all $\mu \in \sigma\left(A_{-1}\right)$. In this case, an infinite number of eigenvalues may belong to the right-half plane. On the other hand, only a finite 
number of eigenvalues may be located on the right of a vertical line $\operatorname{Re} \lambda=\varepsilon$ for any $\varepsilon>0$. For the analysis of stabilizability, it is convenient to construct a decomposition of the state space into three $\mathcal{A}$-invariant subspaces.

We divide the spectrum of $\mathcal{A}$ into three parts:

$$
\sigma(\mathcal{A})=\Lambda_{0} \cup \Lambda_{1} \cup \Lambda_{2}
$$

where the subsets $\Lambda_{0}, \Lambda_{1}, \Lambda_{2}$ are constructed by the following procedure.

Let $N_{0}$ be such that $\lambda_{m}^{k} \in L_{m}^{k}(r), r \leq \frac{1}{3}\left|\widetilde{\lambda}_{m}^{k}-\widetilde{\lambda}_{i}^{j}\right|,(m, k) \neq(i, j)$ for all $k \geq N_{0}$ and for all $m$ such that $\mu_{m} \neq 0$. First, we construct an auxiliary division

$$
\sigma(\mathcal{A})=\chi_{1} \cup \chi_{0}, \quad \chi_{1}=\left\{\lambda_{m}^{k} \in \sigma(\mathcal{A}):|k| \geq N_{0}, m=1, \ldots, \ell, \mu_{m} \neq 0\right\} .
$$

Due to the construction, any vertical strip $\operatorname{St}\left(\delta_{1}, \delta_{2}\right)=\left\{\lambda: \delta_{1}<\operatorname{Re} \lambda<\delta_{2}\right\}$ contains only a finite number of eigenvalues from $\chi_{0}$. We also recall that $\omega_{s}=\sup \{\operatorname{Re} \lambda: \lambda \in \sigma(\mathcal{A})\}<+\infty$.

If $\sigma_{1} \neq \emptyset$ then for any $r>0$ the strip St $(-r, r)$ contains an infinite number of eigenvalues from $\chi_{1}$ and, as we have noticed above, only a finite number of eigenvalues from $\chi_{0}$. Let us fix some $r>0$ and consider the value

$$
\varepsilon=\min _{\lambda \in \operatorname{St}(-r, r) \cap \chi_{0}}|\operatorname{Re} \lambda|
$$

If $\varepsilon>0$, then the vertical strip $\operatorname{St}(-\varepsilon, \varepsilon)$ does not contain eigenvalues from $\chi_{0}$ but contains an infinite number of eigenvalues from $\chi_{1}$. Moreover, the strip $\operatorname{St}(\varepsilon, r)$ contains only a finite number of eigenvalues from $\chi_{1}$. Thus, the strip $\operatorname{St}\left(\varepsilon, \omega_{s}\right)$ contains a finite number of eigenvalues of the operator $\mathcal{A}$ and, therefore, we conclude that these eigenvalues are located in a rectangle $\left\{\lambda: \varepsilon \leq \operatorname{Re} \lambda \leq \omega_{0},|\operatorname{Im} \lambda|<M\right\}$ for some $M>0$. Finally, we put

$$
\begin{aligned}
& \Lambda_{0}=\sigma(\mathcal{A}) \cap\{\lambda: \operatorname{Re} \lambda \leq-\varepsilon\}, \\
& \Lambda_{1}=\sigma(\mathcal{A}) \cap \operatorname{St}(-\varepsilon, \varepsilon), \\
& \Lambda_{2}=\sigma(\mathcal{A}) \cap \operatorname{St}\left(\varepsilon, \omega_{s}\right) .
\end{aligned}
$$

We notice that the relation $\varepsilon=0$ means that there are eigenvalues with a zero real part. In these cases, we calculate $\min _{\lambda \in \operatorname{St}(-r, r) \cap \chi_{0}}|\operatorname{Re} \lambda|$ without taking these eigenvalues into consideration and, after constructing (3.11), we add these eigenvalues to $\Lambda_{2}$.

The obtained sets of eigenvalues may be described as follows: $\Lambda_{0}$ belongs to the left half-plane and is separated from the imaginary axis; $\Lambda_{1}$ consists of an infinite number of simple eigenvalues which may be stable or unstable, the corresponding eigenvectors form a Riesz basis of the closure of their linear span; $\Lambda_{2}$ consists of a finite number of unstable eigenvalues.

Passing now to the construction of invariant subspaces, let us denote the elements of the finite set $\Lambda_{2}$ as $\lambda_{i}$, $i=1, \ldots, r$, the corresponding generalized eigenvectors by $\varphi_{i, j}:\left(\mathcal{A}-\lambda_{i} I\right)^{j} \varphi_{i, j}=0, j=0, \ldots, s_{i}-1$. We also use the notations proposed above: the eigenvalues from $\Lambda_{1}$ are denoted by $\lambda_{m}^{k}$ and the corresponding eigenvectors by $\varphi_{m}^{k}, m=1, \ldots, \ell_{1},|k| \geq N$.

We introduce the following two infinite-dimensional subspaces of eigenvectors:

$$
\begin{aligned}
& M_{2}^{1}=\mathrm{Cl} \operatorname{Lin}\left\{\varphi_{m}^{k}:\left(\mathcal{A}-\lambda_{m}^{k} I\right) \varphi_{m}^{k}=0, \lambda_{m}^{k} \in \Lambda_{1}\right\} \\
& \widehat{M}_{2}^{1}=\mathrm{Cl} \operatorname{Lin}\left\{\psi_{m}^{k}:\left(\mathcal{A}^{*}-\overline{\lambda_{m}^{k}} I\right) \psi_{m}^{k}=0, \lambda_{m}^{k} \in \Lambda_{1}\right\},
\end{aligned}
$$

two finite-dimensional subspaces of eigenvectors and root-vectors:

$$
\begin{aligned}
& M_{2}^{2}=\operatorname{Lin}\left\{\varphi_{i, j}:\left(\mathcal{A}-\lambda_{i} I\right)^{j} \varphi_{i, j}=0, \lambda_{i} \in \Lambda_{2}, j=0, \ldots, s_{i}-1\right\} \\
& \widehat{M}_{2}^{2}=\operatorname{Lin}\left\{\psi_{i, j}:\left(\mathcal{A}^{*}-\overline{\lambda_{i}} I\right)^{j} \psi_{i, j}=0, \lambda_{i} \in \Lambda_{2}, j=0, \ldots, s_{i}-1\right\}
\end{aligned}
$$


and the subspace $M_{2}^{0}$, which satisfies

$$
M_{2}=\left(\widehat{M}_{2}^{1} \oplus \widehat{M}_{2}^{2}\right) \stackrel{\perp}{\oplus} M_{2}^{0} .
$$

Thus, we have constructed three $\mathcal{A}$-invariant subspaces: $M_{2}^{0}, M_{2}^{1}$ and $M_{2}^{2}$. The main result of this subsection is the following theorem.

Theorem 3.4. Let $\sigma_{1}=\left\{\mu_{1}, \ldots, \mu_{\ell_{1}}\right\}$ consist of simple eigenvalues only. For any $N \geq N_{1}$, the decomposition of the spectrum (3.11) and the subspaces given by (3.12)-(3.14) define the direct decomposition of the space $M_{2}$ :

$$
M_{2}=M_{2}^{0} \oplus M_{2}^{1} \oplus M_{2}^{2},
$$

where the subspaces $M_{2}^{0}, M_{2}^{1}, M_{2}^{2}$ are $\mathcal{A}$-invariant.

Proof. The proof of this proposition is very similar to that of Theorem 3.3. We prove that any element $\xi \in M_{2}$ allows the representation:

$$
\xi=\xi_{0}+\sum_{m=1}^{\ell_{1}} \sum_{|k| \geq N} c_{m}^{k} \varphi_{m}^{k}+\sum_{i=1}^{r} \sum_{j=0}^{s_{i}-1} c_{i, j} \varphi_{i, j}, \quad \xi_{0} \in M_{2}^{0}, \quad \sum_{m=1}^{\ell_{1}} \sum_{|k| \geq N}\left|c_{m}^{k}\right|^{2}<\infty .
$$

The eigenvectors $\left\{\varphi_{m}^{k}: \lambda_{m}^{k} \in \Lambda_{1}\right\}$ form a Riesz basis of the closure of their linear span. The finite set of the generalized eigenvectors $\left\{\varphi_{i, j}: \lambda_{i} \in \Lambda_{2}\right\}$ is also a basis of their linear span. These vectors are linearly independent of eigenvectors $\left\{\varphi_{m}^{k}\right\}$. Thus, eigenvectors $\left\{\varphi_{m}^{k}\right\} \cup\left\{\varphi_{i, j}\right\}$ form a basis of the closure of their linear span. Arguing in the same way, we conclude that the set $\left\{\widehat{\psi}_{m}^{k}\right\} \cup\left\{\widehat{\psi}_{i, j}\right\}$ is also a basis of the closure of its linear span, where $\widehat{\psi}_{m}^{k}=\frac{1}{\lambda_{m}^{k}} \psi_{m}^{k}$ and $\widehat{\psi}_{i, j}=\frac{1}{\lambda_{i}} \psi_{i, j}$. Moreover, without loss of generality, we may assume that the sets $\left\{\varphi_{m}^{k}\right\} \cup\left\{\varphi_{i, j}\right\}$ and $\left\{\widehat{\psi}_{m}^{k}\right\} \cup\left\{\widehat{\psi}_{i, j}\right\}$ are biorthogonal after the normalization.

Using the representations (2.5) and (2.6), we choose eigenvectors with $\left\|x_{m}^{k}\right\|_{\mathbb{C}^{n}}=1$ and $\left\|y_{m}^{k}\right\|_{\mathbb{C}^{n}}=1$. Due to Lemma 3.5, there is $C>0$ such that $\left\|\varphi_{m}^{k}\right\|_{M^{2}} \leq C$ and $\left\|\widehat{\psi}_{m}^{k}\right\|_{M^{2}} \leq C$ for all $m=1, \ldots, \ell_{1},|k| \geq N$.

Applying the decomposition (3.4) to vectors $\varphi_{m}^{k}$, we obtain

$$
\varphi_{m}^{k}=\gamma_{m}^{k}+\sum_{i=1}^{\ell_{1}} \sum_{|j| \geq N} a_{m}^{k} \widehat{\psi}_{m}^{k}+\sum_{i=1}^{r} \sum_{j=0}^{s_{i}-1} a_{i, j} \widehat{\psi}_{i, j}, \quad \gamma_{m}^{k} \in M_{2}^{0}
$$

Since the sets $\{\varphi\}$ and $\{\psi\}$ are biorthogonal, the last representation can be rewritten as follows:

$$
\varphi_{m}^{k}=\gamma_{m}^{k}+a_{m}^{k} \widehat{\psi}_{m}^{k}, \quad \gamma_{m}^{k} \in M_{2}^{0}
$$

and, moreover, due to Lemma 2.7 we have

$$
a_{m}^{k}=\frac{\left\langle\varphi_{m}^{k}, \widehat{\psi}_{m}^{k}\right\rangle_{M_{2}}}{\left\|\widehat{\psi}_{m}^{k}\right\|_{M_{2}}^{2}}=\frac{\frac{1}{\lambda_{m}^{k}}\left\langle\varphi_{m}^{k}, \psi_{m}^{k}\right\rangle_{M_{2}}}{\left\|\widehat{\psi}_{m}^{k}\right\|_{M_{2}}^{2}}=\frac{-\frac{1}{\lambda_{m}^{k}}\left\langle\Delta^{\prime}\left(\lambda_{m}^{k}\right) x_{m}^{k}, y_{m}^{k}\right\rangle_{\mathbb{C}^{n}}}{\left\|\widehat{\psi}_{m}^{k}\right\|_{M_{2}}^{2}}
$$

Besides, arguing in the same way, we obtain:

$$
\varphi_{i, j}=\gamma_{i, j}+a_{i, j} \widehat{\psi}_{i, j_{1}}, \quad \gamma_{i, j} \in M_{2}^{0}, j_{1}=0, \ldots, s_{i}-1 .
$$

From (3.15) and (3.16) it also follows that

$$
\left\|\gamma_{m}^{k}\right\| \leq\left\|\varphi_{m}^{k}\right\|+\left|a_{m}^{k}\right|\left\|\widehat{\psi}_{m}^{k}\right\| \leq C+\sqrt{\left|\frac{1}{\lambda}\left\langle\Delta^{\prime}\left(\lambda_{m}^{k}\right) x_{m}^{k}, y_{m}^{k}\right\rangle\right|} .
$$


Using the decomposition (3.4) and the relation (3.15), we represent each vector $\xi \in M_{2}$ as follows:

$$
\begin{aligned}
\xi & =\widehat{\xi}_{0}+\sum_{m=1}^{\ell_{1}} \sum_{|k| \geq N} b_{m}^{k} \widehat{\psi}_{m}^{k}+\sum_{i=1}^{r} \sum_{j=0}^{s_{i}-1} b_{i, j} \widehat{\psi}_{i, j} \\
& =\widehat{\xi}_{0}-\sum_{m=1}^{\ell_{1}} \sum_{|k| \geq N} \frac{b_{m}^{k}}{a_{m}^{k}} \gamma_{m}^{k}-\sum_{i=1}^{r} \sum_{j=0}^{s_{i}-1} \frac{b_{i, j}}{a_{i, j}} \gamma_{i, j}+\sum_{m=1}^{\ell_{1}} \sum_{|k| \geq N} \frac{b_{\lambda}}{a_{m}^{k}} \varphi_{m}^{k}+\sum_{i=1}^{r} \sum_{j=0}^{s_{i}-1} \frac{b_{i, j}}{a_{i, j}} \varphi_{i, j} \\
& =\xi_{0}+\sum_{m=1}^{\ell_{1}} \sum_{|k| \geq N} c_{m}^{k} \varphi_{m}^{k}+\sum_{i=1}^{r} \sum_{j=0}^{s_{i}-1} c_{i, j} \varphi_{i, j}
\end{aligned}
$$

where $\widehat{\xi}_{0} \in M_{2}^{0}, \sum_{m=1}^{\ell_{1}} \sum_{|k| \geq N}\left|b_{m}^{k}\right|^{2}<\infty, \xi_{0}=\widehat{\xi}_{0}-\sum_{m=1}^{\ell_{1}} \sum_{|k| \geq N} \frac{b_{m}^{k}}{a_{m}^{k}} \gamma_{m}^{k}-\sum_{i=1}^{r} \sum_{j=0}^{s_{i}-1} \frac{b_{i, j}}{a_{i, j}} \gamma_{i, j} \in M_{2}^{0}, c_{m}^{k}=\frac{b_{m}^{k}}{a_{m}^{k}}$.

To prove the validity of the decomposition (3.18) it is enough to show that $\left|\frac{1}{a_{m}^{k}}\right| \leq C_{1}$ and $\left\|\gamma_{m}^{k}\right\| \leq C_{2}$. Taking into account (3.16) and (3.17), the latter gives the estimate

$$
0<C_{1} \leq\left|\frac{1}{\lambda_{m}^{k}}\left\langle\Delta^{\prime}\left(\lambda_{m}^{k}\right) x_{m}^{k}, y_{m}^{k}\right\rangle\right| \leq C_{2}, \quad \lambda_{m}^{k} \in \Lambda_{1} .
$$

This estimate is proved by Lemma 3.11. Therefore, the representation (3.18) holds for any $\xi \in M_{2}$, and this completes the proof of the theorem.

\subsection{Auxiliary results}

In this subsection, we prove several estimates which have been used in the proofs of Theorem 3.3 and Theorem 3.4.

Lemma 3.5. Let us consider eigenvectors $\varphi_{m}^{k}=\varphi\left(\lambda_{m}^{k}\right)$ and $\psi_{m}^{k}=\psi\left(\overline{\lambda_{m}^{k}}\right)$ and their representations $(2.5)$ and (2.6). Let us assume that $\left\|x_{m}^{k}\right\|_{\mathbb{C}^{n}}=\left\|y_{m}^{k}\right\|_{\mathbb{C}^{n}}=1$ in these representations. Then, for any $N \in \mathbb{N}$ there is a constant $C>0$ such that

$$
\left\|\varphi_{m}^{k}\right\| \leq C, \quad \frac{1}{\left|\lambda_{m}^{k}\right|}\left\|\psi_{m}^{k}\right\| \leq C, \quad m=1, \ldots, \ell_{1},|k| \geq N .
$$

In other words, the two families of eigenvectors $\left\{\varphi_{m}^{k}: m=1, \ldots, \ell_{1},|k| \geq N\right\}$ with $\left\|x_{m}^{k}\right\|_{\mathbb{C}^{n}}=1$ and $\left\{\frac{1}{\bar{\lambda}_{m}^{k}} \psi_{m}^{k}: m=1, \ldots, \ell_{1},|k| \geq N\right\}$ with $\left\|y_{m}^{k}\right\|_{\mathbb{C}^{n}}=1$ are bounded.

Proof. Using (2.5) and the relation $\left\|x_{m}^{k}\right\|_{\mathbb{C}^{n}}=1$ we obtain:

$$
\begin{aligned}
\left\|\varphi_{m}^{k}\right\|^{2} & =\left\|\left(I-\mathrm{e}^{-\lambda_{m}^{k}} A_{-1}\right) x_{m}^{k}\right\|^{2}+\int_{-1}^{0}\left\|\mathrm{e}^{\lambda_{m}^{k} \theta} x_{m}^{k}\right\|^{2} \mathrm{~d} \theta \\
& \leq\left\|I-\mathrm{e}^{-\lambda_{m}^{k}} A_{-1}\right\|^{2}+\int_{-1}^{0} \mathrm{e}^{2 \operatorname{Re} \lambda_{m}^{k} \theta} \mathrm{d} \theta \\
& \leq 1+\mathrm{e}^{2 \operatorname{Re} \lambda_{m}^{k}}\left\|A_{-1}\right\|^{2}+\frac{1-\mathrm{e}^{-2 \operatorname{Re} \lambda_{m}^{k}}}{2 \operatorname{Re} \lambda_{m}^{k}} \leq C .
\end{aligned}
$$

The last inequality holds since the real function $\frac{1-\mathrm{e}^{-2 s}}{s}$ is bounded in every bounded neighborhood of 0 . This gives the first inequality. 
From (2.6) and since $\left\|y_{m}^{k}\right\|_{\mathbb{C}^{n}}=1$, we have:

$$
\begin{aligned}
\left\|\frac{1}{\lambda_{m}^{k}} \psi_{m}^{k}\right\|^{2}= & \frac{1}{\left|\lambda_{m}^{k}\right|^{2}}\left\|y_{m}^{k}\right\|^{2}+\int_{-1}^{0} \|\left(\mathrm{e}^{-\overline{\lambda_{m}^{k}} \theta}-\frac{1}{\overline{\lambda_{m}^{k}}} A_{2}^{*}(\theta)\right. \\
& \left.+\frac{1}{\overline{\lambda_{m}^{k}}} \mathrm{e}^{-\overline{\lambda_{m}^{k}} \theta} \int_{0}^{\theta} \mathrm{e}^{\overline{\lambda_{m}^{k}} s} A_{3}^{*}(s) \mathrm{d} s+\mathrm{e}^{-\overline{\lambda_{m}^{k}} \theta} \int_{0}^{\theta} \mathrm{e}^{\overline{\lambda_{m}^{k}} s} A_{2}^{*}(s) \mathrm{d} s\right) y_{m}^{k} \|^{2} \mathrm{~d} \theta \\
\leq & \left\|y_{m}^{k}\right\|^{2}\left(\frac{1}{\left|\lambda_{m}^{k}\right|^{2}}+\frac{\mathrm{e}^{2 \operatorname{Re} \lambda_{m}^{k}}-1}{2 \operatorname{Re} \lambda_{m}^{k}}+\frac{1}{\left|\lambda_{m}^{k}\right|^{2}}\left\|A_{2}^{*}(\theta)\right\|_{L_{2}}^{2}\right) \\
& +\int_{-1}^{0} \mathrm{e}^{-2 \operatorname{Re} \lambda_{m}^{k} \theta} \mathrm{d} \theta \int_{-1}^{0} \mathrm{e}^{2 \operatorname{Re} \lambda_{m}^{k} s}\left(\frac{1}{\left|\lambda_{m}^{k}\right|^{2}}\left\|A_{3}^{*}(s)\right\|+\left\|A_{2}^{*}(s)\right\|\right) \mathrm{d} s \\
\leq & \frac{1}{\left|\lambda_{m}^{k}\right|^{2}}+\frac{1}{\left|\lambda_{m}^{k}\right|^{2}}\left\|A_{2}^{*}(\theta)\right\|_{L_{2}}^{2}+\frac{\mathrm{e}^{2 \operatorname{Re} \lambda_{m}^{k}}-1}{2 \operatorname{Re} \lambda_{m}^{k}}\left(1+\frac{1}{\left|\lambda_{m}^{k}\right|^{2}}\left\|A_{3}^{*}(\theta)\right\|_{L_{2}}^{2}+\left\|A_{2}^{*}(\theta)\right\|_{L_{2}}^{2}\right) \\
\leq & \left(\frac{1}{\left|\lambda_{m}^{k}\right|^{2}}+C_{1}\right)\left(\left\|A_{2}^{*}(\theta)\right\|_{L_{2}}^{2}+1\right)+\frac{C_{1}}{\left|\lambda_{m}^{k}\right|^{2}}\left\|A_{3}^{*}(\theta)\right\|_{L_{2}}^{2} \leq C_{2},
\end{aligned}
$$

where $C_{1}$ and $C_{2}$ are some constants.

Remark 3.6. We notice that the norm of eigenvectors $\psi_{m}^{k}$ (assuming $\left\|y_{m}^{k}\right\|=1$ ) increases infinitely when $k \rightarrow \infty$. This can be seen in the example of eigenvectors $\widetilde{\psi}_{m}^{k}$ of the operator $\widetilde{\mathcal{A}}^{*}\left(A_{2}^{*}(\theta)=A_{2}^{*}(\theta) \equiv 0\right)$ :

$$
\begin{aligned}
\left\|\widetilde{\psi}_{m}^{k}\right\|^{2} & =\left\|y_{m}^{k}\right\|^{2}+\int_{-1}^{0}\left\|\overline{\lambda_{m}^{k}} \mathrm{e}^{-\overline{\lambda_{m}^{k}} \theta} y_{m}^{k}\right\|^{2} \mathrm{~d} \theta=\left\|y_{m}^{k}\right\|^{2}\left(1+\left|\lambda_{m}^{k}\right|^{2} \frac{\mathrm{e}^{2 \operatorname{Re} \lambda_{m}^{k}}-1}{2 \operatorname{Re} \lambda_{m}^{k}}\right) \\
& \geq\left(1+C_{3}\left|\lambda_{m}^{k}\right|^{2}\right) \rightarrow+\infty, \quad k \rightarrow \infty,
\end{aligned}
$$

where $C_{3}$ is a constant.

To formulate the next proposition we introduce the matrices

$$
R_{m}=\left(\begin{array}{cc}
\widehat{R}_{m} & 0 \\
0 & I
\end{array}\right), \quad \widehat{R}_{m}=\left(\begin{array}{ccccc}
0 & 0 & \ldots & 0 & 1 \\
0 & 1 & \ldots & 0 & 0 \\
\vdots & \vdots & \ddots & \vdots & \vdots \\
0 & 0 & \ldots & 1 & 0 \\
1 & 0 & \ldots & 0 & 0
\end{array}\right) \in \mathbb{C}^{m \times m}, \quad m=1, \ldots, \ell_{1}
$$

where $I=I_{n-m}$ is the identity matrix of dimension $n-m$. Obviously, $R_{1}=I$ and $R_{m}^{-1}=R_{m}^{*}=R_{m}$ for all $m=1, \ldots, \ell_{1}$.

Lemma 3.7. Assume that $\sigma_{1}=\left\{\mu_{1}, \ldots, \mu_{\ell_{1}}\right\}$ consists of simple eigenvalues only. There exists $N \in \mathbb{N}$ such that for any $\lambda_{m}^{k} \in \Lambda_{1},|k| \geq N$ and the corresponding matrix $\Delta\left(\lambda_{m}^{k}\right)$ there are matrices $P_{m, k}, Q_{m, k}$ of the form

$$
P_{m, k}=\left(\begin{array}{cccc}
1 & -p_{2} & \ldots & -p_{n} \\
0 & 1 & \ldots & 0 \\
\vdots & \vdots & \ddots & \vdots \\
0 & 0 & \ldots & 1
\end{array}\right), \quad Q_{m, k}=\left(\begin{array}{cccc}
1 & 0 & \ldots & 0 \\
-q_{2} & 1 & \ldots & 0 \\
\vdots & \vdots & \ddots & \vdots \\
-q_{n} & 0 & \ldots & 1
\end{array}\right)
$$


such that the product $\frac{1}{\lambda_{m}^{k}} P_{m, k} R_{m} \Delta\left(\lambda_{m}^{k}\right) R_{m} Q_{m, k}$ has the following form:

$$
\frac{1}{\lambda_{m}^{k}} P_{m, k} R_{m} \Delta\left(\lambda_{m}^{k}\right) R_{m} Q_{m, k}=\left(\begin{array}{cccc}
0 & 0 & \ldots & 0 \\
0 & & & \\
\vdots & & S_{m, k} \\
0 & &
\end{array}\right), \quad \operatorname{det} S_{m, k} \neq 0 .
$$

Moreover, for any $\varepsilon>0$, there is $N \in \mathbb{Z}$ such that for any $|k| \geq N$ the components $p_{i}=p_{i}(m, k), q_{i}=q_{i}(m, k)$ of the matrices (3.19) may be estimated as follows:

$$
\left|p_{i}\right| \leq \varepsilon,\left|q_{i}\right| \leq \varepsilon, \quad i=2, \ldots, n
$$

Proof. We begin with the analysis of the structure of the matrix

$$
\frac{1}{\lambda_{m}^{k}} R_{m} \Delta\left(\lambda_{m}^{k}\right) R_{m}=-I+\mathrm{e}^{-\lambda_{m}^{k}} R_{m} A_{-1} R_{m}+\int_{-1}^{0} \mathrm{e}^{\lambda_{m}^{k} s} R_{m}\left(A_{2}(s)+\frac{1}{\lambda_{m}^{k}} A_{3}(s)\right) R_{m} \mathrm{~d} s .
$$

Since the matrix $A_{-1}$ is in Jordan form (2.4), the multiplication of $A_{-1}$ on $R_{m}$ from the left and from the right changes the places of the one-dimensional Jordan blocks $\mu_{1}$ and $\mu_{m}$ :

$$
R_{m} A_{-1} R_{m}=\left(\begin{array}{cc}
\mu_{m} & 0 \\
0 & S
\end{array}\right), \quad S \in C^{(n-1) \times(n-1)} .
$$

We introduce the notation

$$
\int_{-1}^{0} \mathrm{e}^{\lambda s} R_{m}\left(A_{2}(s)+\frac{1}{\lambda} A_{3}(s)\right) R_{m} \mathrm{~d} s=\left(\begin{array}{ccc}
\varepsilon_{11}(\lambda) & \ldots & \varepsilon_{1 n}(\lambda) \\
\vdots & \ddots & \vdots \\
\varepsilon_{n 1}(\lambda) & \ldots & \varepsilon_{n n}(\lambda)
\end{array}\right) .
$$

According to Proposition 4.5, the elements of the matrix (3.22) tend to zero when $|\operatorname{Im} \lambda| \rightarrow \infty($ and $|\operatorname{Re} \lambda| \leq$ $C<\infty)$. Thus, $\left|\varepsilon_{i j}\left(\lambda_{m}^{k}\right)\right| \rightarrow 0$ when $k \rightarrow \infty$.

In the introduced notation, the singular matrix $\frac{1}{\lambda_{m}^{k}} R_{m} \Delta\left(\lambda_{m}^{k}\right) R_{m}$ has the following form:

$$
\frac{1}{\lambda_{m}^{k}} R_{m} \Delta\left(\lambda_{m}^{k}\right) R_{m}=\left(\begin{array}{cccc}
-1+\mathrm{e}^{-\lambda_{m}^{k}} \mu_{m}+\varepsilon_{11}\left(\lambda_{m}^{k}\right) & \varepsilon_{12}\left(\lambda_{m}^{k}\right) & \ldots & \varepsilon_{1 n}\left(\lambda_{m}^{k}\right) \\
\varepsilon_{21}\left(\lambda_{m}^{k}\right) & & & \\
\vdots & S_{m, k} & \\
\varepsilon_{n 1}\left(\lambda_{m}^{k}\right) & &
\end{array}\right)
$$

where

$$
S_{m, k}=-I_{n-1}+\mathrm{e}^{-\lambda_{m}^{k}} S+\left(\begin{array}{ccc}
\varepsilon_{22}\left(\lambda_{m}^{k}\right) & \ldots & \varepsilon_{2 n}\left(\lambda_{m}^{k}\right) \\
\vdots & \ddots & \vdots \\
\varepsilon_{n 2}\left(\lambda_{m}^{k}\right) & \ldots & \varepsilon_{n n}\left(\lambda_{m}^{k}\right)
\end{array}\right)
$$

and $I_{n-1}$ is the identity matrix of dimension $n-1$. Let us prove that

$$
\operatorname{det} S_{m, k} \neq 0 \text {. }
$$


Consider the identity $-I_{n-1}+\mathrm{e}^{-\lambda_{m}^{k}} S=-I_{n-1}+\mathrm{e}^{-\tilde{\lambda}_{m}^{k}} S+\left(\mathrm{e}^{-\lambda_{m}^{k}}-\mathrm{e}^{-\widetilde{\lambda}_{m}^{k}}\right) S$, where $\widetilde{\lambda}_{m}^{k}=\mathrm{i}\left(\arg \mu_{m}+2 \pi k\right)$ is an eigenvalue of the operator $\widetilde{\mathcal{A}}$. Since $\mathrm{e}^{\tilde{\lambda}_{m}^{k}}=\mu_{m}$ we have

$$
-I+\mathrm{e}^{-\tilde{\lambda}_{m}^{k}} R_{m} A_{-1} R_{m}=\left(\begin{array}{cc}
0 & 0 \\
0 & -I_{n-1}+\mathrm{e}^{-\tilde{\lambda}_{m}^{k} S}
\end{array}\right),
$$

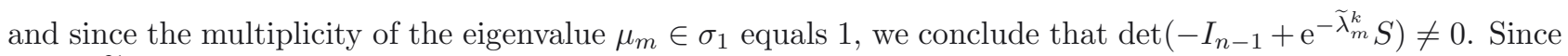
$\left|\lambda_{m}^{k}-\widetilde{\lambda}_{m}^{k}\right| \rightarrow 0$ when $k \rightarrow \infty$, then for any $\varepsilon>0$ there is $N>0$ such that for $k:|k| \geq N$ the estimates $\left|\mathrm{e}^{-\lambda_{m}^{k}}-\mathrm{e}^{-\widetilde{\lambda}_{m}^{k}}\right||| S|| \leq \frac{\varepsilon}{2}$ and $\left|\varepsilon_{i j}\left(\lambda_{m}^{k}\right)\right| \leq \frac{\varepsilon}{2}$ hold. Thus, we have that $\operatorname{det} S_{m, k}=\operatorname{det}\left(-I_{n-1}+\mathrm{e}^{-\widetilde{\lambda}_{m}^{k}} S+B_{m, k}\right)$, where the absolute value of each component of $B_{m, k}$ is less than $\varepsilon$. Therefore, there is $N>0$ such that $S_{m, k}$ is invertible and we obtain the relation (3.24).

Since det $S_{m, k} \neq 0$, from the relation (3.23), we conclude that the first row of the matrix $\frac{1}{\lambda_{m}^{k}} R_{m} \Delta\left(\lambda_{m}^{k}\right) R_{m}$ is a linear combination of all other rows and the first column is a linear combination of all other columns:

$$
\begin{aligned}
& \varepsilon_{1 i}\left(\lambda_{m}^{k}\right)=p_{2} s_{2 i}+\ldots+p_{n} s_{n i}, \quad i=2, \ldots, n, \\
& \varepsilon_{j 1}\left(\lambda_{m}^{k}\right)=q_{2} s_{j 2}+\ldots+q_{n} s_{j n}, \quad j=2, \ldots, n,
\end{aligned}
$$

where $s_{i j}=s_{i j}(m, k), 2 \leq i, j \leq n$ are the components of the matrix $S_{m, k}$.

Let us consider the matrices $P_{m, k}, Q_{m, k}$ of the form (3.19) with the coefficients $p_{2}, \ldots, p_{n}$ and $q_{2}, \ldots, q_{n}$ defined by (3.25). Direct computations give us that $\frac{1}{\lambda_{m}^{k}} P_{m, k} R_{m} \Delta\left(\lambda_{m}^{k}\right) R_{m} Q_{m, k}$ is of the form (3.20), i.e.:

$$
\frac{1}{\lambda_{m}^{k}} P_{m, k} R_{m} \Delta\left(\lambda_{m}^{k}\right) R_{m} Q_{m, k}=\left(\begin{array}{cccc}
0 & 0 & \ldots & 0 \\
0 & & & \\
\vdots & & S_{m, k} & \\
0 & & &
\end{array}\right)
$$

Let us estimate the coefficients $p_{2}, \ldots, p_{n}$ and $q_{2}, \ldots, q_{n}$. The equations (3.25) may be rewritten in the form:

$$
v_{1}=\left(S_{m, k}\right)^{T} w_{1}, \quad v_{2}=S_{m, k} w_{2},
$$

where $v_{1}=\left(\varepsilon_{12}\left(\lambda_{m}^{k}\right), \ldots, \varepsilon_{1 n}\left(\lambda_{m}^{k}\right)\right)^{T}, w_{1}=\left(p_{2}, \ldots, p_{n}\right)^{T}, v_{2}=\left(\varepsilon_{21}\left(\lambda_{m}^{k}\right), \ldots, \varepsilon_{n 1}\left(\lambda_{m}^{k}\right)\right)^{T}, w_{2}=\left(q_{2}, \ldots, q_{n}\right)^{T}$. Since $\operatorname{det} S_{m, k} \neq 0$ then $\quad w_{1}=\left(S_{m, k}^{-1}\right)^{T} v_{1}, \quad w_{2}=S_{m, k}^{-1} v_{2}$

and since the values $\varepsilon_{i j}\left(\lambda_{m}^{k}\right)$ are small then to show (3.21), we have to prove that the estimate $\left\|S_{m, k}^{-1}\right\| \leq C$, $C>0$ holds for all $k:|k| \geq N$.

As we have shown above, $S_{m, k}=-I_{n-1}+\frac{1}{\mu_{m}} S+B_{m, k}$, where elements of the matrices $B_{m, k}$ tend to zero when $k \rightarrow \infty$. Thus, there is $N \in \mathbb{Z}$ such that for all $k:|k| \geq N$ the norm of the matrix $\widetilde{B}_{m, k} \stackrel{\text { def }}{=}-$ $\left(-I_{n-1}+\frac{1}{\mu_{m}} S\right)^{-1} B_{m, k}$ is small enough, say $\left\|\widetilde{B}_{m, k}\right\|<\frac{1}{2}$. Thus, the inverse matrix of $I_{n-1}-\widetilde{B}_{m, k}$ exists for every $|k| \geq N$, and these inverse matrices are bounded uniformly by $k$ :

$$
\left\|\left(I_{n-1}-\widetilde{B}_{m, k}\right)^{-1}\right\|=\left\|\sum_{i=0}^{\infty}\left(\widetilde{B}_{m, k}\right)^{i}\right\| \leq C_{1}, \quad|k| \geq N
$$

Thus, we obtain the estimate

$$
\left\|S_{m, k}^{-1}\right\|=\left\|\left(I_{n-1}-\widetilde{B}_{m, k}\right)^{-1}\left(-I_{n-1}+\frac{1}{\mu_{m}} S\right)^{-1}\right\| \leq C_{1}\left\|\left(-I_{n-1}+\frac{1}{\mu_{m}} S\right)^{-1}\right\| \leq C .
$$

The proof of the lemma is complete. 
Corollary 3.8. The matrix function $\widehat{\Delta}_{m, k}(\lambda) \stackrel{\text { def }}{=} \frac{1}{\lambda} P_{m, k} R_{m} \Delta(\lambda) R_{m} Q_{m, k}$, where $P_{m, k}, Q_{m, k}$ are given by (3.19), enables the following representation in a bounded neighborhood $U\left(\lambda_{m}^{k}\right)$ of the corresponding eigenvalue $\lambda_{m}^{k} \in \Lambda_{1}$, $|k| \geq N$ :

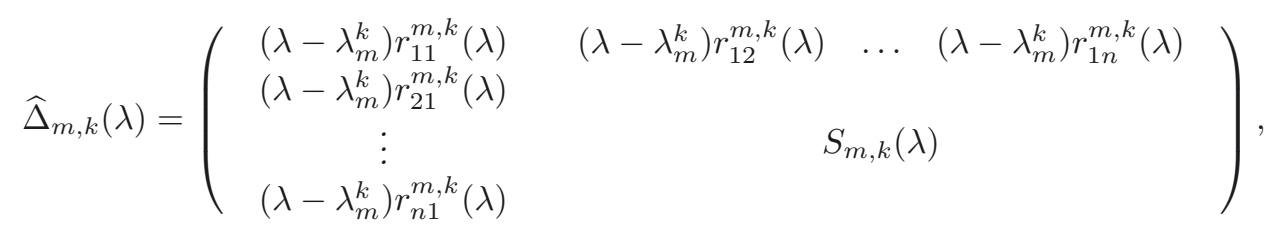

where the functions $r_{i j}^{m, k}(\lambda)$ are analytic in $U\left(\lambda_{m}^{k}\right)$. Moreover,

$$
r_{11}^{m, k}\left(\lambda_{m}^{k}\right) \neq 0, \quad\left|r_{11}^{m, k}\left(\lambda_{m}^{k}\right)\right| \rightarrow 1, k \rightarrow \infty
$$

Proof. Since $\Delta(\lambda)$ is analytic, then all the components of the matrix function

$$
\widehat{\Delta}_{m, k}(\lambda)=\frac{1}{\lambda} P_{m, k} R_{m} \Delta(\lambda) R_{m} Q_{m, k}
$$

are analytic in a bounded neighborhood of the point $\lambda_{m}^{k}$. Moreover, since the matrix $\widehat{\Delta}_{m, k}\left(\lambda_{m}^{k}\right)$ has the form (3.20), then we conclude that $\widehat{\Delta}_{m, k}(\lambda)$ is of the form (3.26).

Let us prove the relation (3.27). If we assume that $r_{11}^{m, k}\left(\lambda_{m}^{k}\right)=0$, then $\left(\lambda-\lambda_{m}^{k}\right) r_{11}^{m, k}(\lambda)=\left(\lambda-\lambda_{m}^{k}\right)^{2} \widehat{r}_{11}^{m, k}(\lambda)$, where $\widehat{r}_{11}^{m, k}(\lambda)$ is analytic. The latter implies that the multiplicity of the root $\lambda=\lambda_{m}^{k}$ of the equation $\operatorname{det} \widehat{\Delta}_{m, k}(\lambda)=0$ is greater than or equal to 2, i.e. $\operatorname{det} \widehat{\Delta}_{m, k}(\lambda)=\left(\lambda-\lambda_{m}^{k}\right)^{2} r(\lambda)$, where $r(\lambda)$ is an analytic function. Indeed, decomposing $\operatorname{det} \widehat{\Delta}_{m, k}(\lambda)$ by the elements of the first row, we see that all the terms of this decomposition have the common multiplier $\left(\lambda-\lambda_{m}^{k}\right)^{2}$. Thus, we obtain that the multiplicity of $\lambda=\lambda_{m}^{k}$ as the root of the equation $\operatorname{det} \Delta(\lambda)=0$ is greater than or equal to 2 , and this is in contradiction with the assumption that $\lambda_{m}^{k}$ is an eigenvalue of multiplicity one of the operator $\mathcal{A}$.

Taking into account (3.23) and the form of the transformations (3.20), we see that

$$
\left(\lambda-\lambda_{m}^{k}\right) r_{11}^{m, k}(\lambda)=\left(-1+\mathrm{e}^{-\lambda} \mu_{m}+\varepsilon_{11}(\lambda)\right)-\sum_{i=2}^{n} p_{i} \varepsilon_{i 1}(\lambda)-\sum_{j=2}^{n} q_{j} \varepsilon_{1 j}(\lambda) .
$$

Differentiating (3.28) by $\lambda$ and substituting $\lambda=\lambda_{m}^{k}$, we obtain

$$
r_{11}^{m, k}\left(\lambda_{m}^{k}\right)=-\mathrm{e}^{-\lambda_{m}^{k}} \mu_{m}+\left(\varepsilon_{11}(\lambda)-\sum_{i=2}^{n} p_{i} \varepsilon_{i 1}(\lambda)-\sum_{j=2}^{n} q_{j} \varepsilon_{1 j}(\lambda)\right)_{\lambda=\lambda_{m}^{k}}^{\prime} .
$$

The terms $\left(\varepsilon_{i j}(\lambda)\right)^{\prime}$ are of the form

$$
\left(\varepsilon_{i j}(\lambda)\right)^{\prime}=\int_{-1}^{0} \mathrm{e}^{\lambda s}\left(s A_{2}(s)+\frac{s}{\lambda} A_{3}(s)-\frac{1}{\lambda^{2}} A_{3}(s)\right)_{i j} \mathrm{~d} s,
$$

therefore, due to Proposition 4.5 and Lemma 3.7, we conclude that

$$
\left(\varepsilon_{11}(\lambda)-\sum_{i=2}^{n} p_{i} \varepsilon_{i 1}(\lambda)-\sum_{j=2}^{n} q_{j} \varepsilon_{1 j}(\lambda)\right)_{\lambda=\lambda_{m}^{k}}^{\prime} \rightarrow 0, \quad k \rightarrow \infty .
$$


Since $-\mathrm{e}^{-\lambda_{m}^{k}} \mu_{m} \rightarrow-1$ when $k \rightarrow \infty$, we obtain the relation (3.27) and, in particular, there is a constant $C>0$ and an integer $N$ such that for $|k|>N$ we have

$$
0<C \leq\left|r_{11}^{m, k}\left(\lambda_{m}^{k}\right)\right|
$$

The latter completes the proof of the proposition.

Remark 3.9. The same arguments give that

$$
\left|r_{i 1}^{m, k}\left(\lambda_{m}^{k}\right)\right| \rightarrow 0,\left|r_{1 j}^{m, k}\left(\lambda_{m}^{k}\right)\right| \rightarrow 0, \quad k \rightarrow \infty
$$

for all $i=2, \ldots, n$ and for all $i=2, \ldots, n$.

Proof. Indeed, let us consider $r_{1 j}^{m, k}(\lambda)$ for $j=2, \ldots, n$ and use the fact that $A_{-1}$ is in a Jordan form:

$$
\left(\lambda-\lambda_{m}^{k}\right) r_{1 j}^{m, k}(\lambda)=\varepsilon_{1 j}(\lambda)-\sum_{i=2}^{n} p_{i} \varepsilon_{i j}(\lambda)+p_{j}\left(-1+\mathrm{e}^{-\lambda} \mu\right)+p_{j-1} c
$$

where $\mu \in \sigma\left(A_{-1}\right)$ and the constant $c=0$ if $\mu$ is geometrically simple or, otherwise, $c=1$. Thus, we obtain

$$
r_{1 j}^{m, k}\left(\lambda_{m}^{k}\right)=-p_{j} \mathrm{e}^{-\lambda_{m}^{k}} \mu+\left(\varepsilon_{1 j}(\lambda)-\sum_{i=2}^{n} p_{i} \varepsilon_{i 1}(\lambda)\right)_{\lambda=\lambda_{m}^{k}}^{\prime}
$$

and since $p_{i}=p_{i}(m, k) \rightarrow 0$ when $k \rightarrow \infty$ due to Lemma 3.7, then we conclude that $\left|r_{1 j}^{m, k}\left(\lambda_{m}^{k}\right)\right| \rightarrow 0$ when $k \rightarrow \infty$.

Remark 3.10. Direct computations give:

$$
P_{m, k}^{-1}=\left(\begin{array}{cccc}
1 & p_{2} & \ldots & p_{n} \\
0 & 1 & \ldots & 0 \\
\vdots & \vdots & \ddots & \vdots \\
0 & 0 & \ldots & 1
\end{array}\right), \quad Q_{m, k}^{-1}=\left(\begin{array}{cccc}
1 & 0 & \ldots & 0 \\
q_{2} & 1 & \ldots & 0 \\
\vdots & \vdots & \ddots & \vdots \\
q_{n} & 0 & \ldots & 1
\end{array}\right)
$$

Lemma 3.11. Let $\sigma_{1}=\left\{\mu_{1}, \ldots, \mu_{\ell_{1}}\right\}$ consist of simple eigenvalues only. There are constants $0<C_{1}<C_{2}$, $N \in \mathbb{Z}$ such that for any $\lambda_{m}^{k} \in \Lambda_{1},|k| \geq N$ the following estimate holds:

$$
0<C_{1} \leq\left|\frac{1}{\lambda_{m}^{k}}\left\langle\Delta^{\prime}\left(\lambda_{m}^{k}\right) x_{m}^{k}, y_{m}^{k}\right\rangle\right| \leq C_{2}
$$

where $\Delta^{\prime}(\lambda)=\frac{\mathrm{d}}{\mathrm{d} \lambda} \Delta(\lambda) ; x_{m}^{k}=x\left(\lambda_{m}^{k}\right), y_{m}^{k}=y\left(\bar{\lambda}_{m}^{k}\right)$ are defined by $(2.5)$, (2.6) and $\left\|x_{m}^{k}\right\|=\left\|y_{m}^{k}\right\|=1$.

Proof. First, we prove the estimate (3.30) for eigenvalues $\lambda_{1}^{k} \in \Lambda_{1}$. Since $x_{1}^{k} \in \operatorname{Ker} \Delta\left(\lambda_{1}^{k}\right)$, then

$$
0=\frac{1}{\lambda_{1}^{k}} P_{1, k}^{-1} P_{1, k} \Delta\left(\lambda_{1}^{k}\right) Q_{1, k} Q_{1, k}^{-1} x_{1}^{k}=P_{1, k}^{-1} \widehat{\Delta}_{1, k}\left(\lambda_{1}^{k}\right) Q_{1, k}^{-1} x_{1}^{k},
$$

where $P_{1, k}, Q_{1, k}, \widehat{\Delta}_{1, k}\left(\lambda_{1}^{k}\right)$ are defined by (3.19), (3.20). Thus, $Q_{1, k}^{-1} x_{1}^{k} \in \operatorname{Ker} \widehat{\Delta}_{1, k}\left(\lambda_{1}^{k}\right)$ and, taking into account the form (3.20) of the matrix $\widehat{\Delta}_{1, k}\left(\lambda_{1}^{k}\right)$, we conclude that $Q_{1, k}^{-1} x_{1}^{k}=\left(\widehat{x}_{1}, 0, \ldots, 0\right)^{T}, \widehat{x}_{1} \neq 0$. On the other hand, applying directly $Q_{1, k}^{-1}$ given by $(3.29)$ to the vector $x_{1}^{k}=\left(\left(x_{1}^{k}\right)_{1}, \ldots,\left(x_{1}^{k}\right)_{n}\right)^{T}$, we conclude that $\widehat{x}_{1}=\left(x_{1}^{k}\right)_{1}$ and $\left(x_{1}^{k}\right)_{i}=-q_{i}\left(x_{1}^{k}\right)_{1}, i=2, \ldots, n$. 
Due to the relation (3.21), for any $\varepsilon>0$ there is $N \in \mathbb{N}$ such that for all $k:|k| \geq N$ we have:

$$
1=\left\|x_{1}^{k}\right\|^{2}=\left|\left(x_{1}^{k}\right)_{1}\right|^{2}\left(1+\left|q_{2}\right|^{2}+\ldots+\left|q_{n}\right|^{2}\right) \leq\left|\left(x_{1}^{k}\right)_{1}\right|^{2}\left(1+(n-1) \varepsilon^{2}\right),
$$

which implies $\left|\left(x_{1}^{k}\right)_{1}\right| \rightarrow 1$ when $k \rightarrow \infty$. Finally, we obtain

$$
Q_{1, k}^{-1} x_{1}^{k}=\left(\left(x_{1}^{k}\right)_{1}, 0, \ldots, 0\right)^{T}, \quad 0<C \leq\left|\left(x_{1}^{k}\right)_{1}\right| \leq 1, \quad|k| \geq N .
$$

Conjugating (3.20), we have the relation

$$
\left(\begin{array}{cccc}
0 & 0 & \ldots & 0 \\
0 & & & \\
\vdots & & S_{1, k}^{*} & \\
0 & &
\end{array}\right)=\left(\frac{1}{\lambda_{1}^{k}} P_{1, k} \Delta\left(\lambda_{1}^{k}\right) Q_{1, k}\right)^{*}=\frac{1}{\overline{\lambda_{1}^{k}}} Q_{1, k}^{*} \Delta^{*}\left(\overline{\lambda_{1}^{k}}\right) P_{1, k}^{*}=\widehat{\Delta}_{1, k}^{*}\left(\overline{\lambda_{1}^{k}}\right) .
$$

Using the fact that $y_{1}^{k} \in \operatorname{Ker} \Delta^{*}\left(\overline{\lambda_{1}^{k}}\right)$ we get:

$$
0=\frac{1}{\overline{\lambda_{1}^{k}}}\left(Q_{1, k}^{*}\right)^{-1} Q_{1, k}^{*} \Delta^{*}\left(\overline{\lambda_{1}^{k}}\right) P_{1, k}^{*}\left(P_{1, k}^{*}\right)^{-1} y_{1}^{k}=\left(Q_{1, k}^{*}\right)^{-1} \widehat{\Delta}_{1, k}^{*}\left(\overline{\lambda_{1}^{k}}\right)\left(P_{1, k}^{*}\right)^{-1} y_{1}^{k}
$$

This gives $\left(P_{1, k}^{*}\right)^{-1} y_{1}^{k} \in \operatorname{Ker} \widehat{\Delta}_{1, k}^{*}\left(\overline{\lambda_{1}^{k}}\right)$ and, taking into account the left-hand side of (3.33), we conclude that $\left(P_{1, k}^{*}\right)^{-1} y_{1}^{k}=\left(\widehat{y}_{1}, 0, \ldots, 0\right)^{T}, \widehat{y}_{1} \neq 0$. Multiplying $\left(P_{1, k}^{*}\right)^{-1}$ on $y_{1}^{k}=\left(\left(y_{1}^{k}\right)_{1}, \ldots,\left(y_{1}^{k}\right)_{1}\right)^{T}$ we obtain the relations $\widehat{y}_{1}=\left(y_{1}^{k}\right)_{1}$ and $\left(y_{1}^{k}\right)_{i}=-\bar{p}_{i}\left(y_{1}^{k}\right)_{1}, i=2, \ldots, n$. Thus, due to (3.21), for any $\varepsilon>0$ and $k:|k| \geq N$ we have:

$$
1=\left\|y_{1}^{k}\right\|^{2}=\left|\left(y_{1}^{k}\right)_{1}\right|^{2}\left(1+\left|\bar{p}_{2}\right|^{2}+\ldots+\left|\bar{p}_{n}\right|^{2}\right) \leq\left|\left(y_{1}^{k}\right)_{1}\right|^{2}\left(1+(n-1) \varepsilon^{2}\right)
$$

and we conclude that $\left|\left(y_{1}^{k}\right)_{1}\right| \rightarrow 1$ when $k \rightarrow \infty$. Finally,

$$
\left(P_{1, k}^{*}\right)^{-1} y_{1}^{k}=\left(\left(y_{1}^{k}\right)_{1}, 0, \ldots, 0\right)^{T}, \quad 0<C \leq\left|\left(y_{1}^{k}\right)_{1}\right| \leq 1,|k| \geq N .
$$

Differentiating (3.26) by $\lambda$ and putting $\lambda=\lambda_{1}^{k}$, we obtain

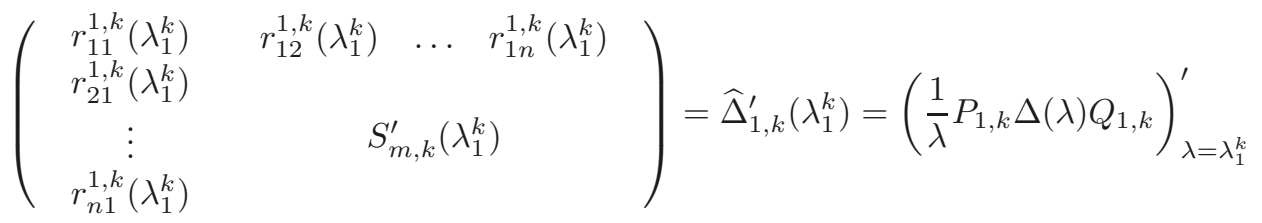

$$
\begin{aligned}
& =P_{1, k}\left(\frac{1}{\lambda_{1}^{k}} \Delta^{\prime}\left(\lambda_{1}^{k}\right)-\frac{1}{\left(\lambda_{1}^{k}\right)^{2}} \Delta\left(\lambda_{1}^{k}\right)\right) Q_{1, k} .
\end{aligned}
$$

Using (3.3) and the relation $x_{1}^{k} \in \operatorname{Ker} \Delta\left(\lambda_{1}^{k}\right)$, we obtain

$$
\begin{aligned}
\frac{1}{\lambda_{1}^{k}}\left\langle\Delta^{\prime}\left(\lambda_{1}^{k}\right) x_{1}^{k}, y_{1}^{k}\right\rangle & =\left\langle P_{1, k}^{-1} P_{1, k}\left(\frac{1}{\lambda_{1}^{k}} \Delta^{\prime}\left(\lambda_{1}^{k}\right)-\frac{1}{\left(\lambda_{1}^{k}\right)^{2}} \Delta\left(\lambda_{1}^{k}\right)\right) Q_{1, k} Q_{1, k}^{-1} x_{1}^{k}, y_{1}^{k}\right\rangle \\
& =\left\langle P_{1, k}\left(\frac{1}{\lambda_{1}^{k}} \Delta^{\prime}\left(\lambda_{1}^{k}\right)-\frac{1}{\left(\lambda_{1}^{k}\right)^{2}} \Delta\left(\lambda_{1}^{k}\right)\right) Q_{1, k} Q_{1, k}^{-1} x_{1}^{k},\left(P_{1, k}^{-1}\right)^{*} y_{1}^{k}\right\rangle \\
& =\left\langle\widehat{\Delta}_{1, k}^{\prime}\left(\lambda_{1}^{k}\right) Q_{1, k}^{-1} x_{1}^{k},\left(P_{1, k}^{-1}\right)^{*} y_{1}^{k}\right\rangle .
\end{aligned}
$$


Finally, using the representation (3.3) of the matrix $\widehat{\Delta}_{1, k}^{\prime}\left(\lambda_{1}^{k}\right)$ and representations (3.32), (3.35) of the vectors $Q_{1, k}^{-1} x_{1}^{k},\left(P_{1, k}^{-1}\right)^{*} y_{1}^{k}$, we conclude that

$$
\frac{1}{\lambda_{1}^{k}}\left\langle\Delta^{\prime}\left(\lambda_{1}^{k}\right) x_{1}^{k}, y_{1}^{k}\right\rangle=r_{11}^{1, k}\left(\lambda_{1}^{k}\right)\left(x_{1}^{k}\right)_{1}\left(y_{1}^{k}\right)_{1}
$$

Moreover, taking into account the estimate (3.27) of Corollary 3.8 and (3.32), (3.35), we obtain the estimate (3.30), which proves the lemma for the case of eigenvalues $\lambda_{1}^{k}$, i.e. for $m=1$.

Let us now prove the estimate (3.30) for $\lambda_{m}^{k} \in \Lambda_{1}, m=2, \ldots, \ell_{1}$. In this case, the idea of the proof remains the same but the argument appears to be more cumbersome. In the proof, we omit some detailed explanations that were given above for the case $m=1$.

Let us consider the product $R_{m} \Delta\left(\lambda_{m}^{k}\right) R_{m}$. Using the relation $x_{m}^{k} \in \operatorname{Ker} \Delta\left(\lambda_{m}^{k}\right)$, we have

$$
0=\frac{1}{\lambda_{m}^{k}} R_{m} P_{m, k}^{-1} P_{m, k} R_{m} \Delta\left(\lambda_{m}^{k}\right) R_{m} Q_{m, k} Q_{m, k}^{-1} R_{m} x_{m}^{k}=R_{m} P_{m, k}^{-1} \widehat{\Delta}_{m, k}\left(\lambda_{m}^{k}\right) Q_{m, k}^{-1} R_{m} x_{m}^{k} .
$$

Thus, $Q_{m, k}^{-1} R_{m} x_{m}^{k} \in \operatorname{Ker} \widehat{\Delta}_{m, k}\left(\lambda_{m}^{k}\right)$ and, from the explicit form (3.20) of $\widehat{\Delta}_{m, k}\left(\lambda_{m}^{k}\right)$, we conclude that $Q_{m, k}^{-1} R_{m} x_{m}^{k}=$ $\left(\widehat{x}_{1}, 0, \ldots, 0\right)^{T}, \widehat{x}_{1} \neq 0$. Multiplying $Q_{m, k}^{-1}$ on $R_{m}$ from the right, we exchange the first and the $m$-th column of $Q_{m, k}^{-1}$, therefore, we obtain:

$$
\left(x_{m}^{k}\right)_{m}=\widehat{x}_{1},\left(x_{m}^{k}\right)_{1}=-q_{m}\left(x_{m}^{k}\right)_{m},\left(x_{m}^{k}\right)_{i}=-q_{i}\left(x_{m}^{k}\right)_{m}, i=2, \ldots, n, i \neq m .
$$

Thus, taking into account (3.21), for any $\varepsilon>0$ there is $N \in \mathbb{N}$ such that for all $k:|k| \geq N$ we have:

$$
1=\left\|x_{m}^{k}\right\|^{2} \leq\left|\left(x_{m}^{k}\right)_{m}\right|^{2}\left(1+(n-1) \varepsilon^{2}\right)
$$

and, thus, $\left|\left(x_{m}^{k}\right)_{m}\right| \rightarrow 1$ when $k \rightarrow \infty$. Therefore,

$$
Q_{m, k}^{-1} R_{m} x_{m}^{k}=\left(\left(x_{m}^{k}\right)_{m}, 0, \ldots, 0\right)^{T}, \quad 0<C \leq\left|\left(x_{m}^{k}\right)_{m}\right| \leq 1,|k| \geq N .
$$

Similar considerations give

$$
\left(P_{m, k}^{-1}\right)^{*} R_{m} y_{m}^{k}=\left(\left(y_{m}^{k}\right)_{m}, 0, \ldots, 0\right)^{T}, \quad 0<C \leq\left|\left(y_{m}^{k}\right)_{m}\right| \leq 1,|k| \geq N .
$$

The derivation of (3.26) by $\lambda$ at $\lambda=\lambda_{m}^{k}$ gives

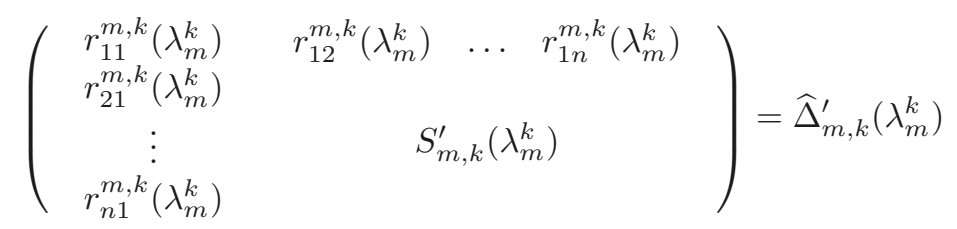

$$
\begin{aligned}
& =P_{m, k} R_{m}\left(\frac{1}{\lambda_{m}^{k}} \Delta^{\prime}\left(\lambda_{m}^{k}\right)-\frac{1}{\left(\lambda_{m}^{k}\right)^{2}} \Delta\left(\lambda_{m}^{k}\right)\right) R_{m} Q_{m, k} .
\end{aligned}
$$


Finally, using (3.40)-(3.3) and the relation $x_{1}^{k} \in \operatorname{Ker} \Delta\left(\lambda_{1}^{k}\right)$, we obtain

$$
\begin{aligned}
\frac{1}{\lambda_{m}^{k}}\left\langle\Delta^{\prime}\left(\lambda_{m}^{k}\right) x_{m}^{k}, y_{m}^{k}\right\rangle & \\
= & \left\langle R_{m} P_{m, k}^{-1} P_{m, k} R_{m}\left(\frac{1}{\lambda_{m}^{k}} \Delta^{\prime}\left(\lambda_{m}^{k}\right)-\frac{1}{\left(\lambda_{m}^{k}\right)^{2}} \Delta\left(\lambda_{m}^{k}\right)\right) R_{m} Q_{m, k} Q_{m, k}^{-1} R_{m} x_{m}^{k}, y_{m}^{k}\right\rangle \\
& =\left\langle\widehat{\Delta}_{m, k}^{\prime}\left(\lambda_{m}^{k}\right) R_{m} Q_{m, k}^{-1} x_{m}^{k},\left(P_{m, k}^{-1}\right)^{*} R_{m} y_{m}^{k}\right\rangle \\
& =r_{11}^{m, k}\left(\lambda_{m}^{k}\right)\left(x_{m}^{k}\right)_{m}\left(y_{m}^{k}\right)_{m} .
\end{aligned}
$$

To complete the proof of the lemma, we use the estimates (3.27), (3.40) and (3.41).

\section{Boundedness of THE RESOLVENT ON INVARIANT SUBSPACES}

In this section, we prove the exponential stability of the restriction of the semigroup $\left\{\mathrm{e}^{t \mathcal{A}}\right\}_{t \geq 0}$ onto $M_{2}^{0}$ (i.e. the semigroup $\left\{\left.\mathrm{e}^{t \mathcal{A}}\right|_{M_{2}^{0}}\right\}_{t \geq 0}$ ), where the invariant subspace $M_{2}^{0}$ is defined in Section 3 by (3.4). To show this, we use the following well-known equivalent condition of exponential stability (see e.g. [35], p. 119 or [17], p. 139):

Let $T(t)$ be a $C_{0}$-semigroup on a Hilbert space $H$ with a generator $A$. Then $T(t)$ is exponentially stable if, and only if, the following conditions hold:

(1) $\{\lambda: \operatorname{Re} \lambda \geq 0\} \subset \rho(A)$;

(2) $\|R(\lambda, A)\| \leq M$ for all $\{\lambda: \operatorname{Re} \lambda \geq 0\}$ and for some constant $M>0$.

Our main result in this Section may be formulated as follows.

Theorem 4.1 (on resolvent boundedness). Let $\sigma_{1}=\left\{\mu_{1}, \ldots, \mu_{\ell_{1}}\right\}$ consist of simple eigenvalues only. On the subspace $M_{2}^{0}$, defined by (3.4), the restriction of the resolvent $\left.R(\lambda, \mathcal{A})\right|_{M_{2}^{0}}$ is uniformly bounded for $\lambda: \operatorname{Re} \lambda \geq 0$ :

$$
\exists C>0, \quad\|R(\lambda, \mathcal{A}) x\| \leq C\|x\|, \quad \forall x \in M_{2}^{0} .
$$

Let us briefly describe the ideas of the proof. From the explicit form of the resolvent (2.1), we conclude that the main difficulty is to prove the uniform boundedness of the term $\Delta^{-1}(\lambda) D(z, \xi, \lambda)$ in bounded neighborhoods of the eigenvalues of $\mathcal{A}$ located close to the imaginary axis. Indeed, since $\operatorname{det} \Delta\left(\lambda_{m}^{k}\right)=0$ for $\lambda_{m}^{k} \in \Lambda_{1}$ and $\operatorname{Re} \lambda_{m}^{k} \rightarrow 0$ when $k \rightarrow \infty$ then the norm of $\Delta^{-1}(\lambda)$ grows infinitely when $\operatorname{Re} \lambda \rightarrow 0$ and $\operatorname{Im} \lambda \rightarrow \infty$ simultaneously. However, the product $\Delta^{-1}(\lambda) D(z, \xi, \lambda)$ turns out to be bounded for $(z, \xi(\cdot)) \in M_{2}^{0}$.

Lemma 4.2. The vector-function $\Delta^{-1}(\lambda) D(z, \xi, \lambda): M_{2}^{0} \times \mathbb{C}^{n} \rightarrow \mathbb{C}^{n}$ is uniformly bounded in bounded neighborhoods $U_{\delta}\left(\lambda_{m}^{k}\right)$ of eigenvalues $\lambda_{m}^{k} \in \Lambda_{1}$ for some fixed $\delta>0$, i.e.:

(1) For any $k:|k|>N$ and $m=1, \ldots, \ell_{1}$ there is a constant $C_{m, k}$ such that the estimate

$$
\left\|\Delta^{-1}(\lambda) D(z, \xi, \lambda)\right\| \leq C_{m, k}\|(z, \xi(\cdot))\|_{M_{2}}
$$

holds for all $\lambda \in U_{\delta}\left(\lambda_{m}^{k}\right)$ and $(z, \xi(\cdot)) \in M_{2}^{0}$;

(2) There is a constant $C>0$ such that $C_{m, k} \leq C$ for all $m=1, \ldots, \ell_{1}, k:|k|>N$.

The proof of this lemma is technically difficult. It essentially uses the following relation.

Lemma 4.3. For any vector $g=(z, \xi(\cdot))^{T} \in M_{2}^{0}$ and for any eigenvalue $\lambda_{m}^{k} \in \Lambda_{1}$ the following relation holds:

$$
D\left(z, \xi, \lambda_{m}^{k}\right) \in \operatorname{Im} \Delta\left(\lambda_{m}^{k}\right) .
$$

The complete proofs of the mentioned propositions are given in the next subsection.

Remark 4.4. Theorem 4.1 also holds for the subspace $M_{2}^{0}$ defined by (3.14) under the assumption $\Lambda_{2}=\emptyset$. The proof remains the same. 


\subsection{The proof of Theorem 4.1}

We begin with several auxiliary propositions.

Proposition 4.5. Let $L_{0} \subset \mathbb{C}$ be a compact set, $f(s) \in L_{2}[-1,0]$ and we denote by $a_{k}(\lambda)=\int_{-1}^{0} \mathrm{e}^{2 \pi \mathrm{i} k s} \mathrm{e}^{\lambda s} f(s) \mathrm{d} s$, $\lambda \in L_{0}, k \in \mathbb{Z}$. Then $a_{k}(\lambda) \rightarrow 0$ when $k \rightarrow \infty$ uniformly on the set $L_{0}$.

Proof. Integrals $a_{k}(\lambda)$ can be considered as Fourier coefficients of the function $\mathrm{e}^{\lambda s} f(s)$, thus, they converge to zero when $k \rightarrow \infty$. We need to prove that they converge uniformly on the set $L_{0}$. We define the mapping $a: L_{0} \rightarrow \ell_{2}$ taking $\lambda \in L_{0}$ to the sequence $a(\lambda)=\left(a_{1}(\lambda), \ldots, a_{k}(\lambda), \ldots\right)$. Indeed, $a(\lambda) \in \ell_{2}$ since $a_{k}(\lambda)$ are Fourier coefficients of the function $\mathrm{e}^{\lambda s} f(s)$. The mapping $a(\lambda)$ is continuous, and thus the image of $L_{0}$ is compact in $\ell_{2}$. This means (see for example [16]) that

$$
\forall \varepsilon>0, \quad \exists N \in \mathbb{N}, \quad \forall n>N, \quad \forall \lambda \in L_{0}, \quad \sum_{k=n+1}^{\infty}\left|a_{k}(\lambda)\right|^{2}<\varepsilon^{2} .
$$

This implies that the convergence $a_{k}(\lambda) \rightarrow 0$ when $k \rightarrow \infty$ is uniform in $L_{0}$.

Corollary 4.6. If the sequence $\left\{\lambda_{k}\right\}$ is such that $\operatorname{Im} \lambda_{k} \rightarrow \infty$ and $-\infty<a \leq \operatorname{Re} \lambda_{k} \leq b<\infty$ then for any $f(s) \in L_{2}\left(0,1 ; \mathbb{C}^{n \times m}\right)$ we have: $\int_{-1}^{0} \mathrm{e}^{\lambda_{k} s} f(s) \mathrm{d} s \rightarrow 0$ when $k \rightarrow \infty$.

Lemma 4.7. Let $U(0)$ be a bounded neighborhood of 0 , then the following estimates hold:

(1) There is a constant $C>0$ such that $\left\|\frac{1}{\lambda} \Delta(\lambda)\right\| \leq C$ for all $\lambda \in\{\lambda: \operatorname{Re} \lambda \geq 0\} \backslash U(0)$, and $\|\Delta(\lambda)\| \leq C$ for all $\lambda \in U(0)$.

(2) There is a constant $C>0$ such that $\left\|\frac{1}{\lambda} D(z, \xi, \lambda)\right\| \leq C\|(z, \xi(\cdot))\|_{M_{2}}$ for all $\lambda \in\{\lambda: \operatorname{Re} \lambda \geq 0\} \backslash U(0)$, and $\|D(z, \xi, \lambda)\| \leq C\|(z, \xi(\cdot))\|_{M_{2}}$ for all $\lambda \in U(0),(z, \xi(\cdot)) \in M_{2}$.

Proof. From the explicit form (2.2) of $\Delta(\lambda)$ we have the estimate

$$
\left\|\frac{1}{\lambda} \Delta(\lambda)\right\| \leq 1+\left\|A_{-1}\right\|+\left\|\int_{-1}^{0} \mathrm{e}^{\lambda s} A_{2}(s) \mathrm{d} s\right\|+\frac{1}{|\lambda|}\left\|\int_{-1}^{0} \mathrm{e}^{\lambda s} A_{3}(s) \mathrm{d} s\right\|
$$

for $\lambda \in\{\lambda: \operatorname{Re} \lambda \geq 0\} \backslash U(0)$. To prove the first item, it is sufficient to show that $\left\|\int_{-1}^{0} \mathrm{e}^{\lambda s} A_{i}(s) \mathrm{d} s\right\|, i=2,3$ are bounded in the same set. Indeed, if we suppose the contrary, then there is an unbounded sequence $\left\{\lambda_{j}\right\}_{j=1}^{\infty}$ such that $\left\|\int_{-1}^{0} \mathrm{e}^{\lambda_{j} s} A_{i}(s) \mathrm{d} s\right\| \rightarrow \infty$ when $j \rightarrow \infty$. On the other hand, it is easy to see that for any $k \geq 0$ : $\int_{-1}^{0} \mathrm{e}^{\lambda s} s^{k} \mathrm{~d} s \rightarrow 0$ when $|\lambda| \rightarrow \infty$ and $\lambda \in\{\lambda: \operatorname{Re} \lambda \geq 0\}$. Since the set of polynomials is dense everywhere in $L_{2}(-1,0)$, then $\left\|\int_{-1}^{0} \mathrm{e}^{\lambda s} A_{i}(s) \mathrm{d} s\right\| \rightarrow 0$ when $|\lambda| \rightarrow \infty, \lambda \in\{\lambda: \operatorname{Re} \lambda \geq 0\}$ and we have come to a contradiction.

This implies that $\left\|\frac{1}{\lambda} \Delta(\lambda)\right\|$ is bounded in the set $\{\lambda: \operatorname{Re} \lambda \geq 0\} \backslash U(0)$. The boundedness of $\|\Delta(\lambda)\|$ in $U(0)$ follows easily from the explicit form (2.2).

The estimates for $D(z, \xi, \lambda)$ may be checked directly, in the same manner, because $\mathrm{e}^{-\lambda} \int_{-1}^{0} \mathrm{e}^{-\lambda s} s^{k} \mathrm{~d} s \rightarrow 0$, when $|\lambda| \rightarrow \infty$ and $\lambda \in\{\lambda: \operatorname{Re} \lambda \geq 0\}, k \geq 0$.

Now we pass to the proofs of the main propositions mentioned at the beginning of the section.

Proof of Lemma 4.2. Let us introduce the following notation:

$$
f(\lambda) \stackrel{\text { def }}{=} \Delta^{-1}(\lambda) D(z, \xi, \lambda)=\left(\frac{1}{\lambda} \Delta(\lambda)\right)^{-1}\left(\frac{1}{\lambda} D(z, \xi, \lambda)\right), \quad(z, \xi(\cdot)) \in M_{2}^{0} .
$$


We analyze the behavior of the vector-function $f(\lambda)$ near the imaginary axis. For the points $\lambda_{m}^{k} \in \Lambda_{1}$, which are the eigenvalues of the operator $\mathcal{A}$, the inverse of the matrix $\Delta\left(\lambda_{m}^{k}\right)$ does not exist. These eigenvalues get closer to the imaginary axis when $k \rightarrow \infty$. Our first aim is to prove that $f(\lambda)$ is bounded in each bounded neighborhood $U\left(\lambda_{m}^{k}\right)$ of $\lambda_{m}^{k} \in \Lambda_{1}$, i.e. that the limit $\lim _{\lambda \rightarrow \lambda_{m}^{k}} \Delta^{-1}(\lambda) D(z, \xi, \lambda)$ exists for all $(z, \xi(\cdot)) \in M_{2}^{0}$.

Since $\Delta(\lambda)$ and $D(z, \xi, \lambda)$ are analytic and since, by construction, all eigenvalues $\lambda_{m}^{k} \in \Lambda_{1}$ are simple, then we have that if $\lambda_{m}^{k}$ is a pole of $f(\lambda)$ then it is a simple pole. In other words, in every bounded neighborhood $U\left(\lambda_{m}^{k}\right)$, the vector-function $f(\lambda)$ may be represented as follows:

$$
f(\lambda)=\frac{1}{\lambda-\lambda_{m}^{k}} f_{-1}+\sum_{i=0}^{\infty}\left(\lambda-\lambda_{m}^{k}\right)^{i} f_{i}
$$

Thus, our aim is to prove that for each $\lambda_{m}^{k}$ the coefficient $f_{-1}=\lim _{\lambda \rightarrow \lambda_{m}^{k}}\left(\lambda-\lambda_{m}^{k}\right) f(\lambda)$ is equal to zero in the representation (4.3), i.e. that $f(\lambda)$ is analytic. To prove this, we construct a representation of the matrix $\left(\frac{1}{\lambda} \Delta(\lambda)\right)^{-1}$ which separates the singularity of this matrix.

According to Lemma 3.5, for each $\lambda_{m}^{k} \in \Lambda_{1}$ there are matrices $P_{m, k}, Q_{m, k}$ such that the value of the matrix-function $\widehat{\Delta}_{m, k}(\lambda)=\frac{1}{\lambda} P_{m, k} R_{m} \Delta(\lambda) R_{m} Q_{m, k}$ at the point $\lambda=\lambda_{m}^{k}$ has the form (3.20), i.e.

$$
\widehat{\Delta}_{m, k}\left(\lambda_{m}^{k}\right)=\frac{1}{\lambda_{m}^{k}} P_{m, k} R_{m} \Delta\left(\lambda_{m}^{k}\right) R_{m} Q_{m, k}=\left(\begin{array}{cccc}
0 & 0 & \ldots & 0 \\
0 & & \\
\vdots & & S_{m, k} \\
0 & &
\end{array}\right), \quad \operatorname{det} S_{m, k} \neq 0 .
$$

We rewrite the representation (4.2) of the function $f(\lambda)$ in a bounded neighborhood $U\left(\lambda_{m}^{k}\right)$ as follows:

$$
\begin{aligned}
f(\lambda) & =\left(\frac{1}{\lambda} R_{m} P_{m, k}^{-1} P_{m, k} R_{m} \Delta(\lambda) R_{m} Q_{m, k} Q_{m, k}^{-1} R_{m}\right)^{-1}\left(\frac{1}{\lambda} D(z, \xi, \lambda)\right) \\
& =R_{m} Q_{m, k}\left(\frac{1}{\lambda} P_{m, k} R_{m} \Delta(\lambda) R_{m} Q_{m, k}\right)^{-1} P_{m, k} R_{m}\left(\frac{1}{\lambda} D(z, \xi, \lambda)\right) \\
& =R_{m} Q_{m, k}\left(\widehat{\Delta}_{m, k}(\lambda)\right)^{-1} P_{m, k} R_{m}\left(\frac{1}{\lambda} D(z, \xi, \lambda)\right) .
\end{aligned}
$$

Let us consider the Taylor expansion of the analytic matrix-function $\widehat{\Delta}_{m, k}(\lambda)$ in $U\left(\lambda_{m}^{k}\right)$ :

$$
\widehat{\Delta}_{m, k}(\lambda)=\widehat{\Delta}_{m, k}\left(\lambda_{m}^{k}\right)+\left(\lambda-\lambda_{m}^{k}\right) \widehat{\Delta}_{m, k}^{\prime}\left(\lambda_{m}^{k}\right)+\sum_{i=2}^{\infty} \frac{1}{i !}\left(\lambda-\lambda_{m}^{k}\right)^{i} \widehat{\Delta}_{m, k}^{(i)}\left(\lambda_{m}^{k}\right) .
$$

Due to Corollary (3.8), $\widehat{\Delta}_{m, k}(\lambda)$ allows the representation (3.26) in some $U\left(\lambda_{m}^{k}\right)$, i.e.

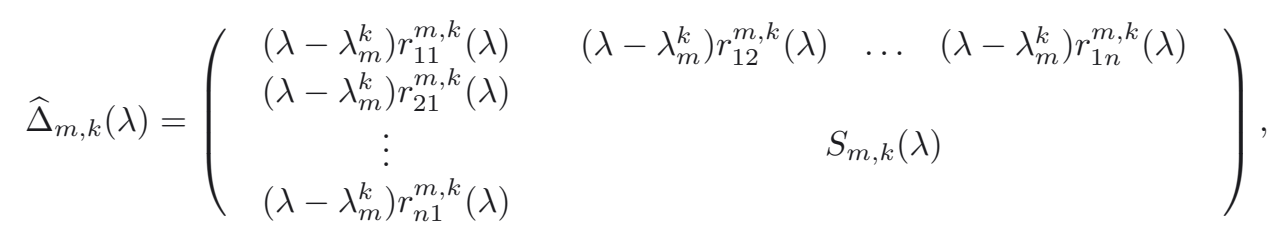


where $r_{i j}^{m, k}(\lambda)$ are analytic functions, and we note that $S_{m, k}\left(\lambda_{m}^{k}\right)=S_{m, k}$, where $S_{m, k}$ is defined by (3.20). Differentiating the last relation by $\lambda$ at $\lambda=\lambda_{m}^{k}$, we obtain:

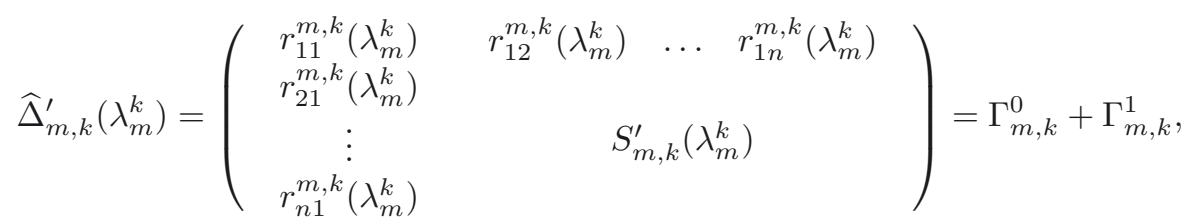

$$
\begin{aligned}
& \Gamma_{m, k}^{0} \stackrel{\text { def }}{=}\left(\begin{array}{cccc}
r_{11}^{m, k}\left(\lambda_{m}^{k}\right) & r_{12}^{m, k}\left(\lambda_{m}^{k}\right) & \ldots & r_{1 n}^{m, k}\left(\lambda_{m}^{k}\right) \\
0 & & 0 & \\
\vdots & & &
\end{array}\right), \quad \Gamma_{m, k}^{1} \stackrel{\text { def }}{=}\left(\begin{array}{cccc}
0 & 0 & \ldots \\
r_{21}^{m, k}\left(\lambda_{m}^{k}\right) & & \\
\vdots & S_{m, k}^{\prime}\left(\lambda_{m}^{k}\right) \\
r_{n 1}^{m, k}\left(\lambda_{m}^{k}\right) &
\end{array}\right) \text {. }
\end{aligned}
$$

We introduce the matrix-function $F_{m, k}(\lambda) \stackrel{\text { def }}{=} \widehat{\Delta}_{m, k}\left(\lambda_{m}^{k}\right)+\left(\lambda-\lambda_{m}^{k}\right) \Gamma_{m, k}^{0}$, which has the following structure:

$$
\left(\begin{array}{cccc}
r_{11}^{m, k}\left(\lambda_{m}^{k}\right)\left(\lambda-\lambda_{m}^{k}\right) & r_{12}^{m, k}\left(\lambda_{m}^{k}\right)\left(\lambda-\lambda_{m}^{k}\right) & \ldots & r_{1 n}^{m, k}\left(\lambda_{m}^{k}\right)\left(\lambda-\lambda_{m}^{k}\right) \\
0 & & & \\
\vdots & & S_{m, k} &
\end{array}\right)
$$

The matrix $F_{m, k}(\lambda)$ is non-singular in a bounded neighborhood $U\left(\lambda_{m}^{k}\right) \backslash\left\{\lambda_{m}^{k}\right\}$. Indeed, due to Lemma 3.5 and Corollary 3.8, we have that $\operatorname{det} S_{m, k} \neq 0, r_{11}^{m, k}\left(\lambda_{m}^{k}\right) \neq 0$, and, thus

$$
\operatorname{det} F_{m, k}(\lambda)=r_{11}^{m, k}\left(\lambda_{m}^{k}\right)\left(\lambda-\lambda_{m}^{k}\right) \operatorname{det} S_{m, k} \neq 0, \quad \lambda \in U\left(\lambda_{m}^{k}\right) \backslash\left\{\lambda_{m}^{k}\right\} .
$$

Therefore, the inverse matrix $F_{m, k}^{-1}(\lambda)$ exists, and is of the following form:

$$
F_{m, k}^{-1}(\lambda)=\left(\begin{array}{cccc}
\frac{1}{r_{11}^{m, k}\left(\lambda_{m}^{k}\right)\left(\lambda-\lambda_{m}^{k}\right)} & F_{21}^{m, k} & \ldots & F_{n 1}^{m, k} \\
0 & F_{22}^{m, k} & \ldots & F_{n 2}^{m, k} \\
\vdots & \vdots & \ddots & \vdots \\
0 & F_{2 n}^{m, k} & \ldots & F_{n n}^{m, k}
\end{array}\right)
$$

where

$$
\begin{array}{rlr}
F_{i 1}^{m, k} & =\frac{1}{r_{11}^{m, k}\left(\lambda_{m}^{k}\right) \operatorname{det} S_{m, k}} \sum_{j=2}^{n}(-1)^{i+j} r_{1 j}^{m, k}\left(\lambda_{m}^{k}\right)\left[S_{m, k}\left(\lambda_{m}^{k}\right)\right]_{i j}, & i=2, \ldots, n, \\
F_{i j}^{m, k} & =(-1)^{i+j}\left[S_{m, k}\left(\lambda_{m}^{k}\right)\right]_{i j}, & i, j=2, \ldots, n,
\end{array}
$$

and by $\left[S_{m, k}(\lambda)\right]_{i j}$ we denote the complementary minor of the element $s_{i j}^{m, k}(\lambda), i, j=2, \ldots, n$ of the matrix $S_{m, k}(\lambda)$. Since the matrix-functions $S_{m, k}(\lambda)$ are analytic and since $S_{m, k}\left(\lambda_{m}^{k}\right) \rightarrow S$ when $k \rightarrow \infty$, then $\left\|S_{m, k}(\lambda)\right\|$, $\left\|\left[S_{m, k}(\lambda)\right]_{i j}\right\|$ and $\left|s_{i j}^{m, k}(\lambda)\right|$ are uniformly bounded for all $k$ and $\lambda \in U_{\delta}\left(\widetilde{\lambda}_{m}^{k}\right)$. Thus, we conclude that $\left|F_{i j}^{m, k}\right| \leq C$ for all $k$ and $i, j=2, \ldots, n$. Moreover, since $r_{1 j}^{m, k}\left(\lambda_{m}^{k}\right) \rightarrow 0$ due to Remark 3.9, then $F_{i 1}^{m, k} \rightarrow 0, i=2, \ldots, n$ when $k \rightarrow \infty$.

Let us rewrite the representation (4.5) as follows:

$$
\begin{aligned}
\widehat{\Delta}_{m, k}(\lambda) & =F_{m, k}(\lambda)+\left(\lambda-\lambda_{m}^{k}\right) \Gamma_{m, k}^{1}+\sum_{i=2}^{\infty} \frac{1}{i !}\left(\lambda-\lambda_{m}^{k}\right)^{i} \widehat{\Delta}_{m, k}^{(i)}\left(\lambda_{m}^{k}\right) \\
& =F_{m, k}(\lambda)\left(I+\left(\lambda-\lambda_{m}^{k}\right) F_{m, k}^{-1}(\lambda) \Gamma_{m, k}^{1}+\sum_{i=2}^{\infty} \frac{1}{i !}\left(\lambda-\lambda_{m}^{k}\right)^{i} F_{m, k}^{-1}(\lambda) \widehat{\Delta}_{m, k}^{(i)}\left(\lambda_{m}^{k}\right)\right)
\end{aligned}
$$


and introduce the notation

$$
\Upsilon_{m, k}(\lambda) \stackrel{\text { def }}{=}\left(\lambda-\lambda_{m}^{k}\right) F_{m, k}^{-1}(\lambda) \Gamma_{m, k}^{1}+\sum_{i=2}^{\infty} \frac{1}{i !}\left(\lambda-\lambda_{m}^{k}\right)^{i} F_{m, k}^{-1}(\lambda) \widehat{\Delta}_{m, k}^{(i)}\left(\lambda_{m}^{k}\right) .
$$

Let us prove that for any $\varepsilon>0$ there is $\delta>0$ and $N \in \mathbb{N}$ such that for any $k:|k|>N$ the following estimate holds:

$$
\left\|\Upsilon_{m, k}(\lambda)\right\| \leq \varepsilon, \quad \lambda \in U_{\delta}\left(\widetilde{\lambda}_{m}^{k}\right) .
$$

From (4.8) we have that $\Upsilon_{m, k}(\lambda)=F_{m, k}^{-1}(\lambda) \widehat{\Delta}_{m, k}(\lambda)-I$, and we are proving the estimate $\| F_{m, k}^{-1}(\lambda) \widehat{\Delta}_{m, k}(\lambda)-$ $I \| \leq \varepsilon, \lambda \in U_{\delta}\left(\widetilde{\lambda}_{m}^{k}\right)$. Using the representations (3.26), (4.6) and (4.7), we estimate the elements $\left\{\gamma_{i j}^{m, k}(\lambda)\right\}_{i, j=1}^{n}$ of the matrix $\Upsilon_{m, k}(\lambda)$. For the sake of convenience, we divide these elements into several groups and we begin with the element $\gamma_{11}^{m, k}(\lambda)$ :

$$
\gamma_{11}^{m, k}(\lambda)=\frac{r_{11}^{m, k}(\lambda)}{r_{11}^{m, k}\left(\lambda_{m}^{k}\right)}+\left(\lambda-\lambda_{m}^{k}\right) \sum_{i=2}^{n} F_{i 1}^{m, k} r_{i 1}^{m, k}(\lambda)-1
$$

Due to Corollary 3.8 and since $r_{11}^{m, k}(\lambda)$ is analytic, there is $\delta>0$ and $N \in \mathbb{N}$ such that $\left|\frac{r_{11}^{m, k}(\lambda)}{r_{11}^{m, k}\left(\lambda_{m}^{k}\right)}\right|<\frac{\varepsilon}{2 n}$ for all $k:|k|>N$ and $\lambda \in U_{\delta}\left(\widetilde{\lambda}_{m}^{k}\right)$. Besides, since $F_{i 1}^{m, k} \rightarrow 0, i=2, \ldots, n$ and $r_{i 1}^{m, k}\left(\lambda_{m}^{k}\right) \rightarrow 0$ when $k \rightarrow \infty$, we obtain the estimate $\left|\gamma_{11}^{m, k}(\lambda)\right|<\frac{\varepsilon}{n}$ for all $k:|k|>N, \lambda \in U_{\delta}\left(\widetilde{\lambda}_{m}^{k}\right)$.

Let us consider other diagonal elements of the matrix $\Upsilon_{m, k}(\lambda)$ :

$$
\gamma_{j j}^{m, k}(\lambda)=\sum_{i=2}^{n} F_{i j}^{m, k} s_{i j}^{m, k}(\lambda)-1=\sum_{i=2}^{n}(-1)^{i+j}\left[S_{m, k}(\lambda)\right]_{i j}\left(s_{i j}^{m, k}(\lambda)-s_{i j}^{m, k}\left(\lambda_{m}^{k}\right)\right), \quad j=2, \ldots, n .
$$

There is $\delta>0$ such that $\left|\gamma_{j j}^{m, k}(\lambda)\right|<\frac{\varepsilon}{n}$ for all $k:|k|>N, \lambda \in U_{\delta}\left(\widetilde{\lambda}_{m}^{k}\right)$. Further, we consider the elements of the first row:

$$
\gamma_{1 j}^{m, k}(\lambda)=\frac{r_{1 j}^{m, k}(\lambda)}{r_{11}^{m, k}\left(\lambda_{m}^{k}\right)}+\sum_{i=2}^{n} F_{i 1}^{m, k} s_{i j}^{m, k}(\lambda), \quad j=2, \ldots, n .
$$

Since $F_{i 1}^{m, k} \rightarrow 0, i=2, \ldots, n$ and $r_{1 j}^{m, k}\left(\lambda_{m}^{k}\right) \rightarrow 0$ when $k \rightarrow \infty$, we obtain the estimate $\left|\gamma_{1 j}^{m, k}(\lambda)\right|<\frac{\varepsilon}{n}$ for all $k:|k|>N, \lambda \in U_{\delta}\left(\widetilde{\lambda}_{m}^{k}\right)$.

Next, we consider all other elements:

$$
\gamma_{i j}^{m, k}(\lambda)=\sum_{r=2}^{n} F_{r i}^{m, k} s_{r j}^{m, k}(\lambda)=\sum_{r=2}^{n}(-1)^{i+r}\left[S_{m, k}(\lambda)\right]_{i r}\left(s_{r j}^{m, k}(\lambda)-s_{r j}^{m, k}\left(\lambda_{m}^{k}\right)\right), i, j=2, \ldots, n ; i \neq j .
$$

They may be estimated as $\left|\gamma_{i j}^{m, k}(\lambda)\right|<\frac{\varepsilon}{n}$ for all $k:|k|>N, \lambda \in U_{\delta}\left(\widetilde{\lambda}_{m}^{k}\right)$ choosing small enough $\delta>0$.

Finally, we obtain the estimate (4.9), and then there is $\delta>0, N \in \mathbb{N}$ such that the matrix $I+\Upsilon_{m, k}(\lambda)$ has an inverse for any $\lambda \in U_{\delta}\left(\widetilde{\lambda}_{m}^{k}\right), k:|k|>N$ :

$$
\left(I+\Upsilon_{m, k}(\lambda)\right)^{-1}=I+\left(\lambda-\lambda_{m}^{k}\right) \Gamma_{m, k}(\lambda),
$$

where $\Gamma_{m, k}(\lambda)$ is analytic in a bounded neighborhood $U_{\delta}\left(\lambda_{m}^{k}\right)$. 
Lastly, from (4.4), (4.8) and (4.10) we obtain:

$$
\begin{aligned}
f(\lambda) & =R_{m} Q_{m, k} \widehat{\Delta}_{m, k}^{-1}(\lambda) P_{m, k} R_{m}\left(\frac{1}{\lambda} D(z, \xi, \lambda)\right) \\
& =R_{m} Q_{m, k}\left(F_{m, k}(\lambda)\left(I+\Upsilon_{m, k}(\lambda)\right)\right)^{-1} R_{m} P_{m, k}\left(\frac{1}{\lambda} D(z, \xi, \lambda)\right) \\
& =R_{m} Q_{m, k}\left(I+\left(\lambda-\lambda_{m}^{k}\right) \Gamma_{m, k}(\lambda)\right) F_{m, k}^{-1}(\lambda) P_{m, k} R_{m}\left(\frac{1}{\lambda} D(z, \xi, \lambda)\right) .
\end{aligned}
$$

Let us now use the fact that $(z, \xi(\cdot)) \in M_{2}^{0}$. In Lemma 4.3 the important relation $D\left(z, \xi, \lambda_{m}^{k}\right) \in \operatorname{Im} \Delta\left(\lambda_{m}^{k}\right)$, $(z, \xi(\cdot)) \in M_{2}^{0}$ is stated. Since in the definition of $\widehat{\Delta}_{m, k}(\lambda)$ the matrices $P_{m, k}, Q_{m, k}, R_{m}$ are nonsingular, then

$$
\frac{1}{\lambda_{m}^{k}} P_{m, k} R_{m} D\left(z, \xi, \lambda_{m}^{k}\right) \in \operatorname{Im} \widehat{\Delta}_{m, k}\left(\lambda_{m}^{k}\right) .
$$

Moreover, since the matrix $\widehat{\Delta}_{m, k}\left(\lambda_{m}^{k}\right)$ is of the form (3.20), we conclude that the first component of the vector $\frac{1}{\lambda_{m}^{k}} P_{m, k} R_{m} D\left(z, \xi, \lambda_{m}^{k}\right)$ equals zero:

$$
\frac{1}{\lambda_{m}^{k}} P_{m, k} R_{m} D\left(z, \xi, \lambda_{m}^{k}\right) \stackrel{\text { def }}{=} \widehat{d}_{m, k}=\left(0, c_{2}, \ldots, c_{n}\right)^{T}
$$

and since the vector-function $\frac{1}{\lambda} P_{m, k} R_{m} D(z, \xi, \lambda)$ is analytic in a bounded neighborhood $U\left(\lambda_{m}^{k}\right)$, we conclude that

$$
\frac{1}{\lambda} P_{m, k} R_{m} D(z, \xi, \lambda)=\widehat{d}_{m, k}+d_{m, k}(\lambda), \quad d_{m, k}\left(\lambda_{m}^{k}\right)=0 .
$$

Finally, we note that $F_{m, k}^{-1}(\lambda) \widehat{d}_{m, k}$ is a constant vector and the vector-function $F_{m, k}^{-1}(\lambda) d_{m, k}(\lambda)$ is bounded in $U\left(\lambda_{m}^{k}\right)$. Taking into account (4.11), (4.6) and (4.12), we obtain that

$$
\begin{aligned}
\lim _{\lambda \rightarrow \lambda_{m}^{k}}\left(\lambda-\lambda_{m}^{k}\right) f(\lambda) & =R_{m} Q_{m, k} \lim _{\lambda \rightarrow \lambda_{m}^{k}}\left(\lambda-\lambda_{m}^{k}\right) F_{m, k}^{-1}(\lambda) P_{m, k} R_{m}\left(\frac{1}{\lambda} D(z, \xi, \lambda)\right) \\
& =R_{m} Q_{m, k} \lim _{\lambda \rightarrow \lambda_{m}^{k}}\left(\lambda-\lambda_{m}^{k}\right) F_{m, k}^{-1}(\lambda)\left(\widehat{d}_{m, k}+d_{m, k}(\lambda)\right) \\
& =0 .
\end{aligned}
$$

Thus, we have proved that $f(\lambda)=\Delta^{-1}(\lambda) D(z, \xi, \lambda)$ is an analytic vector-function in $U_{\delta}\left(\lambda_{m}^{k}\right), k:|k|>N$, $m=1, \ldots, \ell_{1}$ and this gives the estimate $\left\|\Delta^{-1}(\lambda) D(z, \xi, \lambda)\right\| \leq C_{m, k},(z, \xi(\cdot)) \in M_{2}^{0}$.

We have now to prove that $f(\lambda)$ is uniformly bounded in the neighborhoods $U_{\delta}\left(\lambda_{m}^{k}\right)$ for all $k:|k|>N$, $m=1, \ldots, \ell_{1}$. In other words, we must prove that the set of vectors

$$
f_{0}=f_{0}^{m, k}=f\left(\lambda_{m}^{k}\right)=\left(\Delta^{-1}(\lambda) D(z, \xi, \lambda)\right)_{\lambda=\lambda_{m}^{k}}
$$

is bounded. Taking into account the representation (4.11), we obtain:

$$
\begin{aligned}
f_{0}^{m, k} & =\left(R_{m} Q_{m, k} F_{m, k}^{-1}(\lambda) P_{m, k} R_{m}\left(\frac{1}{\lambda} D(z, \xi, \lambda)\right)\right)_{\lambda=\lambda_{m}^{k}} \\
& =R_{m} Q_{m, k} F_{m, k}^{-1}(\lambda)\left(\widehat{d}_{m, k}+d_{m, k}(\lambda)\right)_{\lambda=\lambda_{m}^{k}} \\
& =R_{m} Q_{m, k}\left(\frac{d_{1}^{1}}{r_{11}^{m, k}\left(\lambda_{m}^{k}\right)}+\sum_{i=2}^{n} c_{i} F_{i 1}^{m, k}, \sum_{i=2}^{n} c_{i} F_{i 2}^{m, k}, \ldots, \sum_{i=2}^{n} c_{i} F_{i n}^{m, k}\right)^{T},
\end{aligned}
$$

where $d_{1}^{1}=\left(d_{m, k}^{\prime}\left(\lambda_{m}^{k}\right)\right)_{1}$ is the first component of the derivative of $d_{m, k}(\lambda)$ at the point $\lambda_{m}^{k}$. 
As we have mentioned above, there is a constant $C_{1}>0$ such that $\left\|F_{i j}^{m, k}\right\| \leq C_{1}$, for all $k \in \mathbb{N}, m=1, \ldots, \ell_{1}$. The estimates $\left\|P_{m, k}\right\| \leq C_{1}$ and $\left\|Q_{m, k}\right\| \leq C_{1}$ follow from the estimate (3.21) of Lemma 3.7. The estimates $\left|c_{i}\right|<C_{1}$ and $\left|d_{1}^{1}\right|<C_{1}$ follow immediately from Lemma 4.7. From the relation (3.27) of Corollary 3.8, it follows that there is a constant $C_{2}>0$ such that $0<C_{2} \leq\left|r_{11}^{m, k}\left(\lambda_{m}^{k}\right)\right|$ for all $k \in \mathbb{N}, m=1, \ldots, \ell_{1}$ and, thus,

$$
\frac{1}{\left|r_{11}^{m, k}\left(\lambda_{m}^{k}\right)\right|} \leq \frac{1}{C_{2}}
$$

Finally, we conclude that $\left\|f_{0}^{m, k}\right\| \leq C$ for all $m=1, \ldots, \ell_{1}, k:|k| \geq N$, and this completes the proof of the lemma.

Proof of Lemma 4.3. Since $g \in M_{2}^{0}$ then $g \perp \psi_{m}^{k}=\psi\left(\overline{\lambda_{m}^{k}}\right)$ for all $\lambda_{m}^{k} \in \Lambda_{1}$. Therefore, the proposition follows from Lemma 2.10.

Proof of Theorem 4.1. Let $\delta>0$ be such that Lemma 4.2 holds. We divide the closed right half-plane into the following two sets:

$$
\begin{aligned}
& K_{1}(\delta)=\left\{\lambda: \operatorname{Re} \lambda \geq 0, \lambda \in U_{\delta}\left(\widetilde{\lambda}_{m}^{k}\right), \lambda_{m}^{k} \in \Lambda_{1}\right\} \\
& K_{2}(\delta)=\{\lambda: \operatorname{Re} \lambda \geq 0\} \backslash K_{1} .
\end{aligned}
$$

First, let us estimate $\|R(\lambda, \mathcal{A}) x\|$ for any $\lambda \in K_{1}(\delta)$ and $x \in M_{2}^{0}$. Due to Lemma 4.2, we have:

$$
\left\|\Delta^{-1}(\lambda) D(z, \xi, \lambda)\right\| \leq C_{1}\|x\|, \quad x=(z, \xi(\cdot)) \in M_{2}^{0} .
$$

Due to Corollary 4.6, we have the estimate $\left\|\int_{-1}^{0} \mathrm{e}^{-\lambda s} \xi(s) \mathrm{d} s\right\| \leq C_{2}\|x\|$. Thus, for any $x=(z, \xi(\cdot)) \in M_{2}^{0}$, $\lambda \in K_{1}(\delta)$ we obtain:

$$
\begin{aligned}
\|R(\lambda, \mathcal{A}) x\|= & \left\|\mathrm{e}^{-\lambda} A_{-1} \int_{-1}^{0} \mathrm{e}^{-\lambda s} \xi(s) \mathrm{d} s+\left(I-\mathrm{e}^{-\lambda} A_{-1}\right) \Delta^{-1}(\lambda) D(z, \xi, \lambda)\right\|_{\mathbb{C}^{n}} \\
& +\left\|\int_{0}^{\theta} \mathrm{e}^{\lambda(\theta-s)} \xi(s) \mathrm{d} s+\mathrm{e}^{\lambda \theta} \Delta^{-1}(\lambda) D(z, \xi, \lambda)\right\|_{L_{2}} \\
\leq & \mathrm{e}^{\delta}\left\|A_{-1}\right\| C_{2}\|x\|+\left(1+\mathrm{e}^{\delta}\left\|A_{-1}\right\|\right) C_{1}\|x\| \\
& +\left(\int_{-1}^{0}\left\|\int_{0}^{\theta} \mathrm{e}^{\lambda(\theta-s)} \xi(s) \mathrm{d} s+\mathrm{e}^{\lambda \theta} \Delta^{-1}(\lambda) D(z, \xi, \lambda)\right\|_{\mathbb{C}^{n}}^{2} \mathrm{~d} \theta\right)^{\frac{1}{2}} \\
\leq & {\left[\mathrm{e}^{\delta}\left\|A_{-1}\right\| C_{2}+\left(1+\mathrm{e}^{\delta}\left\|A_{-1}\right\|\right) C_{1}+\left(\mathrm{e}^{\delta} C_{2}+C_{1}^{2}\right)^{\frac{1}{2}}\right]\|x\|=C\|x\| . }
\end{aligned}
$$

Let us consider $\lambda \in K_{2}(\delta)$. There is $\varepsilon>0$ such that $\left|\frac{1}{\lambda} \operatorname{det} \Delta(\lambda)\right| \geq \varepsilon$ for any $\lambda \in K_{2}(\delta) \backslash U(0)$. Indeed, if we suppose the contrary then there is a sequence $\left\{\lambda_{i}\right\}_{i=1}^{\infty}$ such that $\left|\frac{1}{\lambda_{i}} \operatorname{det} \Delta\left(\lambda_{i}\right)\right| \rightarrow 0, i \rightarrow \infty$. If the sequence $\left\{\lambda_{i}\right\}_{i=1}^{\infty}$ is bounded, then it contains a converging subsequence: $\lambda_{i_{j}} \rightarrow \widehat{\lambda}$ and, thus, $\left|\frac{1}{\lambda} \operatorname{det} \Delta(\hat{\lambda})\right|=0$. However, the closure of the set $K_{2}(\delta) \backslash U(0)$ does not contain zeros of the function $\operatorname{det} \Delta(\lambda)$ and we have obtained a contradiction.

If the sequence $\left\{\lambda_{i}\right\}_{i=1}^{\infty}$ is unbounded, it contains a subsequence $\lambda_{i_{j}}$ such that $\left|\lambda_{i_{j}}\right| \rightarrow \infty$ when $j \rightarrow \infty$, but we also have

$$
\int_{-1}^{0} \mathrm{e}^{\lambda_{i_{j}} s} A_{2}(s) \mathrm{d} s \rightarrow 0, \quad \frac{1}{\lambda_{i_{j}}} \int_{-1}^{0} \mathrm{e}^{\lambda_{i_{j}} s} A_{3}(s) \mathrm{d} s \rightarrow 0, \quad i \rightarrow \infty .
$$


Moreover, since $\left|\operatorname{det}\left(-I+\mathrm{e}^{-\lambda} A_{-1}\right)\right|=\left|\prod\left(1+\mathrm{e}^{-\lambda} \mu_{m}\right)\right| \geq \prod\left|1+\mathrm{e}^{\delta} \mu_{m}\right|$, we conclude that

$$
\left|\frac{1}{\lambda} \operatorname{det} \Delta\left(\lambda_{i}\right)\right|=\operatorname{det}\left(-I+\mathrm{e}^{-\lambda_{i}} A_{-1}+\int_{-1}^{0} \mathrm{e}^{\lambda_{i} s} A_{2}(s) \mathrm{d} s+\frac{1}{\lambda_{i}} \int_{-1}^{0} \mathrm{e}^{\lambda_{i} s} A_{3}(s) \mathrm{d} s \nrightarrow 0, \quad i \rightarrow \infty .\right.
$$

Thus, we have obtained a contradiction again.

Taking into account the estimates $\left\|\frac{1}{\lambda} \Delta(\lambda)\right\| \leq C_{1}$ and $\left\|\frac{1}{\lambda} D(z, \xi, \lambda)\right\| \leq C_{2}\|x\|$ from Lemma 4.7, we conclude that $\left\|\Delta^{-1}(\lambda) D(z, \xi, \lambda)\right\| \leq C_{3}\|x\|$ for all $\lambda \in K_{2}(\delta) \backslash U(0)$. It is easy to see that $\left\|\mathrm{e}^{-\lambda} \int_{-1}^{0} \mathrm{e}^{-\lambda s} \xi(s) \mathrm{d} s\right\| \leq C_{4}\|x\|$ for all $\lambda \in K_{2}(\delta) \backslash U(0)$. Finally, similarly to (4.13) we obtain the following estimate

$$
\|R(\lambda, \mathcal{A}) x\| \leq C\|x\|, \quad \lambda \in K_{2} .
$$

This completes the proof of the theorem.

\section{Stability ANALYSis}

Based on the results from Sections 3 and 4, we prove the main result on stability which does not assume the condition $\operatorname{det} A_{-1} \neq 0$.

Theorem 5.1. If $\sigma(\mathcal{A}) \subset\{\lambda: \operatorname{Re} \lambda<0\}$ and $\sigma_{1}=\sigma\left(A_{-1}\right) \cap\{\mu:|\mu|=1\}$ consists only of simple eigenvalues, then the system (1.4) is strongly asymptotically stable.

Proof. Let us show that $\left\|\mathrm{e}^{t \mathcal{A}} x\right\| \rightarrow 0$ when $t \rightarrow+\infty$ for any $x \in M_{2}$. Due to Theorem 3.3, each $x \in M_{2}$ allows the following representation:

$$
x=x_{0}+x_{1}, \quad x_{0} \in M_{2}^{0}, x_{1} \in M_{2}^{1},
$$

where $M_{2}^{0}$ and $M_{2}^{1}$ are defined by (3.2)-(3.4). Moreover, the basis of $M_{2}^{1}$ consists of the following eigenvectors:

$$
\left\{\varphi_{m}^{k}:\left(\mathcal{A}-\lambda_{m}^{k} I\right) \varphi_{m}^{k}=0, \lambda_{m}^{k} \in \Lambda_{1}=\Lambda_{1}(N)\right\}
$$

Thus, for any $x_{1} \in M_{2}^{1}$ we have the representations

$$
x_{1}=\sum_{m=1}^{\ell_{1}} \sum_{|k| \geq N} c_{m}^{k} \varphi_{m}^{k}, \quad \mathrm{e}^{t \mathcal{A}} x_{1}=\sum_{m=1}^{\ell_{1}} \sum_{|k| \geq N} \mathrm{e}^{\lambda_{m}^{k} t} c_{m}^{k} \varphi_{m}^{k},
$$

where $\sum_{m=1}^{\ell_{1}} \sum_{|k| \geq N}\left|c_{m}^{k}\right|^{2}<\infty$. Let us consider a norm $\|\cdot\|_{1}$ in which the Riesz basis (5.1) is orthogonal, then we have the following estimate:

$$
\left\|\mathrm{e}^{t \mathcal{A}} x_{1}\right\|_{1}=\left(\sum_{m=1}^{\ell_{1}} \sum_{|k| \geq N} \mathrm{e}^{2 \operatorname{Re} \lambda_{m}^{k} t}\left\|c_{m}^{k} \varphi_{m}^{k}\right\|_{1}^{2}\right)^{\frac{1}{2}} \leq\left\|x_{1}\right\|_{1} .
$$

Since the series $\sum_{m=1}^{\ell_{1}} \sum_{|k| \geq N} c_{m}^{k} \varphi_{m}^{k}$ converges and since $\left\|\varphi_{m}^{k}\right\|_{1} \leq C$ for all $k$ and $m=1, \ldots, \ell_{1}$, then for any $\varepsilon>0$ there is $N_{1} \geq N$ such that $\sum_{m=1}^{\ell_{1}} \sum_{|k| \geq N_{1}}\left\|c_{m}^{k} \varphi_{m}^{k}\right\|_{1}^{2} \leq \frac{\varepsilon^{2}}{8}$. Moreover, since the set $\left\{(m, k): m=1, \ldots, \ell_{1}\right.$, 
$\left.N \leq|k| \leq N_{1}\right\}$ is finite and since $\operatorname{Re} \lambda_{m}^{k}<0$, then there is $t_{0}>0$ such that for any $t \geq t_{0}$ we have: $\sum_{m=1}^{\ell_{1}} \sum_{N \leq|k| \leq N_{1}} \mathrm{e}^{2 \operatorname{Re} \lambda_{m}^{k} t}\left\|c_{m}^{k} \varphi_{m}^{k}\right\|_{1}^{2} \leq \frac{\varepsilon^{2}}{8}$. Thus, we obtain

$$
\sum_{m=1}^{\ell_{1}} \sum_{|k| \geq N} \mathrm{e}^{2 \operatorname{Re} \lambda_{m}^{k} t}\left\|c_{m}^{k} \varphi_{m}^{k}\right\|_{1}^{2} \leq \sum_{m=1}^{\ell_{1}} \sum_{N \leq|k| \leq N_{1}} \mathrm{e}^{2 \operatorname{Re}{ }_{m}^{k} \lambda t}\left\|c_{m}^{k} \varphi_{m}^{k}\right\|_{1}^{2}+\sum_{m=1}^{\ell_{1}} \sum_{|k| \geq N_{1}}\left\|c_{m}^{k} \varphi_{m}^{k}\right\|_{1}^{2} \leq \frac{\varepsilon^{2}}{4} .
$$

Due to Theorem 4.1, the semigroup $\left.\mathrm{e}^{t \mathcal{A}}\right|_{M_{2}^{0}}$ is exponentially stable, i.e. by definition there are some positive constants $M, \omega$ such that $\left\|\left.\mathrm{e}^{t \mathcal{A}}\right|_{M_{2}^{0}}\right\| \leq M \mathrm{e}^{-\omega t}$. Thus, for any $x_{0} \in M_{2}^{0}$, there is $t_{0}>0$ such that for any $t \geq t_{0}$ we have an estimate

$$
\left\|\mathrm{e}^{t \mathcal{A}} x_{0}\right\|_{1} \leq M \mathrm{e}^{-\omega t}\left\|x_{0}\right\|_{1} \leq \frac{\varepsilon}{2} .
$$

Finally, from the estimates (5.2)-(5.4), we conclude that for any $x \in M_{2}$ and for any $\varepsilon>0$ there is $t_{0}>0$ such that for any $t \geq t_{0}$ the following estimate holds:

$$
\left\|\mathrm{e}^{t \mathcal{A}} x\right\|_{1} \leq\left\|\mathrm{e}^{t \mathcal{A}} x_{0}\right\|_{1}+\left\|\mathrm{e}^{t \mathcal{A}} x_{1}\right\|_{1} \leq \varepsilon .
$$

Therefore, $\lim _{t \rightarrow+\infty}\left\|\mathrm{e}^{t \mathcal{A}} x\right\|_{1}=0$, i.e. the system (1.5) is strongly asymptotically stable.

Remark 5.2. One can easily see that under the assumptions of Theorem 5.1 and if the set $\{\mu:|\mu|=1\}$ is not empty then the index $\omega_{0}(\mathcal{A})=0$. This, in particular, means (see for example [30]) that the norm of the semigroup $\left\|\mathrm{e}^{t A}\right\| \geq 1, t \geq 0$ and, as a consequence, there are some solutions $\mathrm{e}^{t A} x$ with an arbitrarily slow rate of decay. On the other hand, one can prove a uniform estimation of this rate from the above if we consider the initial data only on some non-closed subspaces of $M_{2}$, for example, if $x$ belongs to $\mathcal{D}(\mathcal{A})$ or $\mathcal{D}\left(\mathcal{A}^{\alpha}\right), \alpha>0$. Such an approach is considered, for example, in $[2,5]$, where polynomial estimates of the rate of decay of semigroups are obtained under some assumption concerning the behavior of the resolvent of the generators. In the present work, we do not consider this problem. However, we can remark that, in our case, the existence of the polynomial estimates similar to those given in $[2,5]$ may be described in terms of the rate of deviation of eigenvalues of $\mathcal{A}$ from the imaginary axis. Such problems are under investigation.

\subsection{An example of a dilemma: stable and unstable situations}

In this subsection, we give an explicit example illustrating the item (iii) of Theorem 1.1. Namely, we construct two systems having the same spectrum and satisfying the following conditions: $\sigma(\mathcal{A}) \subset\{\lambda: \operatorname{Re} \lambda<0\}$ and there are no Jordan blocks, corresponding to eigenvalues from $\sigma_{1}=\sigma\left(A_{-1}\right) \cap\{\mu:|\mu|=1\}$, but there is an eigenvalue $\mu \in \sigma_{1}$ whose eigenspace is at least two-dimensional. Moreover, one of the constructed systems appears to be stable while the other is unstable.

We consider the system of the form

$$
\dot{z}(t)=\left(\begin{array}{rr}
-1 & 0 \\
0 & -1
\end{array}\right) \dot{z}(t-1)+\left(\begin{array}{rr}
-b & s \\
0 & -b
\end{array}\right) z(t), \quad z \in \mathbb{C}^{2}, \quad t \geq 0,
$$

where $b$ is a real positive number and for the value of $s$ we essentially distinguish two cases: $s=0$ and $s \neq 0$.

The eigenvalues of the operator $\mathcal{A}$ are the roots of the equation $\operatorname{det} \Delta_{\mathcal{A}}(\lambda)=0$, which, in our particular case, has the form:

$$
\operatorname{det}\left(-\lambda I+\lambda \mathrm{e}^{-\lambda} A_{-1}+A_{0}\right)=\operatorname{det}\left(\begin{array}{cc}
-\lambda-\lambda \mathrm{e}^{-\lambda}-b & s \\
0 & -\lambda-\lambda \mathrm{e}^{-\lambda}-b
\end{array}\right)=0 .
$$

Thus, all the eigenvalues of the operator $\mathcal{A}$ satisfy the equation

$$
\lambda \mathrm{e}^{\lambda}+\lambda+b \mathrm{e}^{\lambda}=0
$$


and the multiplicity of each eigenvalue equals two. To prove that $\sigma(\mathcal{A}) \subset\{\lambda: \operatorname{Re} \lambda<0\}$, we use the results on transcendental equations obtained by Pontryagin [23]. It is easy to show that if $s=0$ then the operator $\mathcal{A}$ possesses eigenvectors only, i.e. it possesses no root vectors; if $s \neq 0$ then, to any eigenvalue $\lambda \in \sigma(\mathcal{A})$, there correspond an eigenvector and a root vector of the operator $\mathcal{A}$. This implies that if $s=0$ then the system (5.5) is stable, and if $s \neq 0$ it is unstable.

\section{Stabilizability By REgular FEedBaCK}

In this section, we prove Theorem 1.2 for control systems (1.7) with $\operatorname{det} A_{-1}=0$. It is convenient to reformulate this theorem as follows.

Theorem 6.1 (on stabilizability). Let $b_{1}, \ldots, b_{p} \in \mathbb{C}^{n}$ be the columns of the matrix B. Assume that the following four conditions are satisfied:

(1) all the eigenvalues of the matrix $A_{-1}$ satisfy $|\mu| \leq 1$;

(2) all the eigenvalues $\mu \in \sigma_{1}$ are simple;

(3) $\sum_{i=1}^{p} \mid\left\langle b_{i}, y\right\rangle_{\mathbb{C}^{n}} \neq 0$ for all $i=1, \ldots, p$ and all vectors $y$ satisfying $y \in \operatorname{Ker} \Delta_{\mathcal{A}}^{*}(\lambda)$ for roots $\lambda$ of the equation $\operatorname{det} \Delta_{\mathcal{A}}^{*}(\lambda)=0$, such that $\operatorname{Re} \lambda \geq 0$;

(4) $\sum_{i=1}^{p} \mid\left\langle b_{i}, y_{m}\right\rangle_{\mathbb{C}^{n}} \neq 0$ for all vectors $y_{m}$ of the matrix $A_{-1}^{*}$, corresponding to eigenvalues $\bar{\mu}_{m}, \mu_{m} \in \sigma_{1}$ and for all $i=1, \ldots, p$.

Then there is a regular control $u=\mathcal{F} x$ of the form

$$
u=\mathcal{F} x=\int_{-1}^{0} F_{2}(\theta) \dot{z}_{t}(\theta) \mathrm{d} \theta+\int_{-1}^{0} F_{3}(\theta) z_{t}(\theta) \mathrm{d} \theta,
$$

where $x=(y, z(\cdot)) \in \mathcal{D}(\mathcal{A}), F_{2}(\cdot), F_{3}(\cdot) \in L_{2}\left(-1,0 ; \mathbb{C}^{n \times p}\right)$. Moreover, this control stabilizes the system (1.7), i.e. $\mathcal{D}(\mathcal{A})=\mathcal{D}(\mathcal{A}+\mathcal{B} \mathcal{F})$ and $\mathrm{e}^{t(\mathcal{A}+\mathcal{B F})} x_{0} \rightarrow 0$ as $t \rightarrow \infty$ for all $x_{0} \in M_{2}$.

The controllability conditions (3)-(4) of Theorem 6.1 are equivalent to (3)-(4) of Theorem 1.2 (for more details concerning such conditions see [26]). We also note that regular stabilizability for a particular case of the control systems (1.7) had been considered in [24,25]. Before giving the proof, let us discuss the conditions (1) and (2). Since the regular feedback does not change the matrix $A_{-1}$ we need the assumption $\sigma\left(A_{-1}\right) \subset\{\mu:|\mu| \leq 1\}$. Moreover, taking into account the results of Theorem 5.1 on strong stability, we conclude that by means of a regular feedback it is possible to stabilize the system when the algebraic multiplicity of each eigenvalue $\mu \in \sigma_{1}$ equals 1. In this case, the closed loop system will be asymptotically stable if and only if all the eigenvectors of $\mathcal{A}+\mathcal{B F}$ are in the left half-plane. Thus, the problem of regular stabilizability for such systems consists in assigning the spectrum of the system in the left half-plane. The most intensional problem in the situation appears when an infinite number of the eigenvalues located "close" to the imaginary axis belong to the right half-plane. This means, in particular, that $\sigma_{1} \neq \emptyset$.

Proof of Theorem 6.1. Let us construct the decomposition of the spectrum of $\mathcal{A}$ introduced in Section 3.2 by (3.10):

$$
\sigma(\mathcal{A})=\Lambda_{0}(\mathcal{A}) \cup \Lambda_{1}(\mathcal{A}) \cup \Lambda_{2}(\mathcal{A})
$$

where the subsets are given by (3.11). Since $\Lambda_{0}(\mathcal{A}) \subset\{\lambda: \operatorname{Re} \lambda \leq-\varepsilon\}, \varepsilon>0$ then our aim is to construct the feedback which moves the eigenvalues of $\Lambda_{1}(\mathcal{A})$ and $\Lambda_{2}(\mathcal{A})$ to the left half-plane.

We begin by moving the spectral set $\Lambda_{2}(\mathcal{A})$. The spectral decomposition of the state space $M_{2}=M_{2}^{0} \oplus M_{2}^{1} \oplus$ $M_{2}^{2}$ (Thm. 3.4), corresponding to (6.2), allows us to rewrite the system (1.8) as

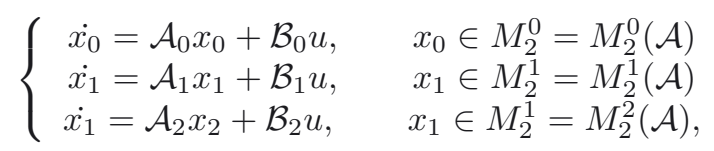

where $\mathcal{A}_{0}=\left.\mathcal{A}\right|_{M_{2}^{0}}, \mathcal{A}_{1}=\left.\mathcal{A}\right|_{M_{2}^{1}}, \mathcal{A}_{2}=\left.\mathcal{A}\right|_{M_{2}^{2}}$ and $\mathcal{B}_{i}$ are the projections of $\mathcal{B}$ onto subspaces $M_{2}^{i}$. 
The spectrum $\Lambda_{2}(\mathcal{A})$ of the operator $\mathcal{A}_{2}$ defined on the finite-dimensional subspace $M_{2}^{2}$ belongs to the right half-plane. Due to the assumption (3) of the theorem all the eigenvalues of $\mathcal{A}_{2}$ are controllable and, thus, they may be assigned arbitrarily to the half-plane $\{\lambda: \operatorname{Re} \lambda \leq-\varepsilon\}$. Namely, let us consider a feedback $u=\mathcal{F}_{2} x$, $\mathcal{F}_{2}: M_{2} \rightarrow \mathbb{C}^{p}$ which acts as follows:

$$
\mathcal{F}_{2} x_{0}=\mathcal{F}_{2} x_{1}=0, x_{0} \in M_{2}^{0}, x_{1} \in M_{2}^{1}, \quad \mathcal{F}_{2} x_{2}=\widehat{\mathcal{F}}_{2} x_{2}, x_{2} \in M_{2}^{2},
$$

where $\widehat{\mathcal{F}}: M_{2}^{2} \rightarrow \mathbb{C}^{p}$ is a bounded operator. It is easy to see that such a type of feedback allows only eigenvalues of $\Lambda_{2}(\mathcal{A})$ to be moved, i.e. the set $\Lambda_{0}(\mathcal{A}) \cup \Lambda_{1}(\mathcal{A})$ remains in $\sigma\left(\mathcal{A}+\mathcal{B} \mathcal{F}_{2}\right)$. Thus, we conclude that there is $\widehat{\mathcal{F}}$ such that the spectrum of the closed-loop system $\mathcal{A}+\mathcal{B F}_{2}$ is of the form

$$
\sigma\left(\mathcal{A}+\mathcal{B F}_{2}\right)=\sigma(\mathcal{A}) \backslash \Lambda_{2}(\mathcal{A}) \cup \widehat{\Lambda}_{2}, \quad \widehat{\Lambda}_{2} \subset\{\lambda: \operatorname{Re} \lambda \leq-\varepsilon\} .
$$

We choose the control in the form $u=\mathcal{F}_{2} x+v$, denote $\widehat{\mathcal{A}} \stackrel{\text { def }}{=} \mathcal{A}+\mathcal{B F}_{0}$ and rewrite the system (1.8) as

$$
\dot{x}=\widehat{\mathcal{A}} x+\mathcal{B} v .
$$

We emphasize, that due to the form of the feedback $\mathcal{F}_{2}$, the infinite-dimensional system (6.4) corresponds to the neutral type system (1.7) with the same matrix $A_{-1}$.

Let us now construct the decomposition of the spectrum (3.10) for the operator $\widehat{\mathcal{A}}$. Due to $(6.3)$, we obtain $\Lambda_{2}(\widehat{\mathcal{A}})=\emptyset$ which gives

$$
\sigma(\widehat{\mathcal{A}})=\Lambda_{0}(\widehat{\mathcal{A}}) \cup \Lambda_{1}(\widehat{\mathcal{A}}), \quad \Lambda_{1}(\widehat{\mathcal{A}})=\Lambda_{1}(\mathcal{A}) .
$$

Applying Theorem 3.4, we construct the spectral decomposition of the state space $M_{2}=M_{2}^{0} \oplus M_{2}^{1}$. The operator model (6.4) may be rewritten as follows:

$$
\left\{\begin{array}{cl}
\dot{x_{0}}=\widehat{\mathcal{A}}_{0} x_{0}+\widehat{\mathcal{B}}_{0} v, & x_{0} \in M_{2}^{0}=M_{2}^{0}(\widehat{\mathcal{A}}) \\
\dot{x_{1}}=\widehat{\mathcal{A}}_{1} x_{1}+\widehat{\mathcal{B}}_{1} v, & x_{1} \in M_{2}^{1}=M_{2}^{1}(\widehat{\mathcal{A}})
\end{array}\right.
$$

where $\widehat{\mathcal{A}}_{0}=\left.\widehat{\mathcal{A}}\right|_{M_{2}^{0}}, \widehat{\mathcal{A}}_{1}=\left.\widehat{\mathcal{A}}\right|_{M_{2}^{1}}$ and $\widehat{\mathcal{B}}_{i}$ are the projections of the vectors of $\mathcal{B}$ onto subspaces $M_{2}^{i}$.

Due to Theorem 4.4, the restriction of the resolvent $\left.R(\lambda, \widehat{\mathcal{A}})\right|_{M_{2}^{0}}$ is uniformly bounded on the set $\{\lambda: \operatorname{Re} \lambda \geq 0\}$ and, therefore, the semigroup $\left\{\left.\mathrm{e}^{t \widehat{\mathcal{A}}}\right|_{M_{2}^{0}}\right\}_{t \geq 0}$ is exponentially stable.

To stabilize the second equation, we apply the approach introduced in [29] which is based on the abstract theorem on infinite pole assignment. Below, for the sake of completeness and due to the specific form of the operator $\widehat{\mathcal{A}}_{1}$, we give a simplified formulation of the mentioned theorem (Thm. 6.5).

The theorem on infinite pole assignment holds for a single input system. However, as is shown in [29], the multivariable case may be reduced to the single input case by the following considerations (see [29] and also the classical result on finite-dimensional control systems in [38]).

Let us consider the vector $\mathbf{b}$ given by

$$
\mathbf{b}=\left(\begin{array}{c}
b \\
0
\end{array}\right)=\left(\begin{array}{c}
c_{1} b_{1}+\ldots+c_{p} b_{p} \\
0
\end{array}\right)=\left(\begin{array}{c}
B \\
0
\end{array}\right) c=\mathcal{B} c, \quad c=\left(\begin{array}{c}
c_{1} \\
\vdots \\
c_{p}
\end{array}\right)
$$

where $\left\{b_{1}, \ldots, b_{p}\right\} \subset \mathbb{C}^{n}$ are the columns of the matrix $B$. There are numbers $c_{i} \in \mathbb{C}$ such that the following relations hold (Lem. 6.6):

(a) $\left\langle b, y_{m}\right\rangle_{\mathbb{C}^{n}} \neq 0$ for all eigenvectors $y_{m}$ of the matrix $A_{-1}^{*}$ corresponding to the eigenvalues $\overline{\mu_{m}}$, where $\mu_{m} \in \sigma_{1}, m=1, \ldots, \ell_{1}$

(b) $\left\langle b, y_{m}^{k}\right\rangle_{\mathbb{C}^{n}} \neq 0$ for all such $y_{m}^{k}$ which satisfy $y_{m}^{k} \in \operatorname{Ker} \Delta^{*}\left(\overline{\lambda_{m}^{k}}\right)$ and $\operatorname{Re} \lambda_{m}^{k} \geq 0$. 
Let us denote the projection of $\mathbf{b}$ onto the subspace $M_{2}^{1}$ by $\mathbf{b}_{1}$ and consider the single input system

$$
\dot{x_{1}}=\widehat{\mathcal{A}}_{1} x_{1}+\mathbf{b}_{1} \widehat{u}, \quad \widehat{u} \in \mathbb{C} .
$$

Due to the construction, the eigenvalues of $\widehat{\mathcal{A}}_{1}$ are simple and each eigenvalue $\lambda_{m}^{k}, m=1, \ldots, \ell_{1},|k| \geq N_{1}$ belongs to the circle $L_{m}^{k}\left({ }^{(k)}\right)$ centered at $\widetilde{\lambda}_{m}^{k}=\mathrm{i}\left(\arg \mu_{m}+2 \pi k\right)$. The corresponding eigenvectors $\varphi_{m}^{k}$ form a Riesz basis (Prop. 2.8).

Thus, the single input system (6.6) satisfies the conditions (H1)-(H4) of Theorem 6.5. According to that theorem, the spectrum $\Lambda_{1}(\mathcal{A})$ of the operator $\widehat{\mathcal{A}_{1}}$ may be moved by the regular feedback (6.1) as follows.

Let us chose scalars $\widehat{\lambda}_{m}^{k}$ such that $\operatorname{Re} \widehat{\lambda}_{m}^{k}<0$ and which are located inside the circles $L_{m}^{k}\left(r^{(k)}\right)$. There is a feedback $\widehat{u}=\widehat{\mathcal{F}}_{1} x, \widehat{\mathcal{F}}_{1}: M_{2} \rightarrow \mathbb{C}$ such that $\widehat{\lambda}_{m}^{k}$ are eigenvalues of the operator $\widehat{\mathcal{A}}_{1}+\mathbf{b}_{1} \widehat{\mathcal{F}}_{1}$. Taking into account (6.5), we define the feedback $v=\mathcal{F}_{1} x, \mathcal{F}_{1}: M_{2} \rightarrow \mathbb{C}^{p}$ as follows

$$
F_{1} x \stackrel{\text { def }}{=} c \widehat{\mathcal{F}}_{1} x .
$$

Due to the theorem on stability, the semigroup $\left\{\left.\mathrm{e}^{t\left(\widehat{\mathcal{A}}+\mathcal{B F}_{1}\right)}\right|_{M_{2}^{1}}\right\}_{t \geq 0}$ is asymptotically stable. Thus, the feedback

$$
u=\mathcal{F}_{1} x_{1}+\mathcal{F}_{2} x_{2}
$$

transforms the original system into one where all the conditions of Theorem 5.1 on asymptotic stability are verified. The latter completes the proof of Theorem 6.1.

Remark 6.2. We would like to emphasize that, in the present paper, to prove the result on stabilizability for the case $\operatorname{det} A_{-1}=0$ we have contributed those ideas which are mainly technical in character: the direct decomposition of the state space and the proof of the resolvent boundedness on some subspace. However, the main contribution from the stabilizability point of view is the abstract theorem on infinite pole assignment which has been proved in [29].

Remark 6.3. The stabilizability of the restriction of the system onto the subspace $M_{2}^{2}(\mathcal{A})$ also follows from some classical results. Let us denote by $\Gamma_{\delta}$ a rectifiable, simple, closed curve which surrounds the spectral set $\Lambda_{2}(\mathcal{A})$ and $\Gamma_{\delta} \subset\{\lambda: \operatorname{Re} \lambda \geq \varepsilon\}$. By $P_{2}=\frac{1}{2 \pi \mathrm{i}} \int_{\Gamma_{\delta}} R(\mathcal{A}, \lambda) \mathrm{d} \lambda$ we denote the spectral projector. The subspaces $P_{2} M_{2}$ and $\left(I-P_{2}\right) M_{2}$ are $\mathcal{A}$-invariant and the spectrum of the restriction $\left(I-P_{2}\right) M_{2}$ belongs to the half-plane $\{\lambda: \operatorname{Re} \lambda<\varepsilon\}$. According to [34], the spectral set $\Lambda_{2}(\mathcal{A})$ may be assigned arbitrarily to the half-plane $\{\lambda: \operatorname{Re} \lambda<\varepsilon\}$ by means of a finite rank input operator (under some controllability conditions). The development of this approach was given in [13,19] (see e.g. [8] for a review).

Remark 6.4. Let us discuss the assumptions (3)-(4) of Theorem 1.2:

(3) $\operatorname{rank}(\Delta(\lambda), B)=n$ for all $\lambda: \operatorname{Re} \lambda \geq 0$.

(4) $\operatorname{rank}\left(\mu I-A_{-1}, B\right)=n$ for all $\mu \in \sigma_{1}$.

The assumption (3) may include an infinite number of relations (obviously, it should be verified only for $\lambda$ which are eigenvalues of $\mathcal{A}$, i.e. $\operatorname{det} \Delta_{\mathcal{A}}(\lambda)=0$; there may be an infinite number of eigenvalues of $\mathcal{A}$ belonging to the right half-plane). However, only a finite number of these relations have to be verified. More precisely, there is $M>0$ such that for any eigenvalue $\lambda$ such that $|\operatorname{Im} \lambda|>M$ the condition (3) follows from the condition (4).

Proof. Let $A_{-1}$ be of the form (2.4) and we prove the proposition for eigenvalues located inside the circles $L_{1}^{k}\left(r^{(k)}\right)$, i.e. for $\lambda_{1}^{k}$ (for all other indices $m=1, \ldots, \ell_{1}$ the idea of the proof remains the same). The matrix $A_{-1}-\mu_{1} I=A_{-1}-\mathrm{e}^{-\tilde{\lambda}_{1}^{k}} I$ is of the form:

$$
A_{-1}-\mu_{1} I=\left(\begin{array}{cc}
0 & 0 \\
0 & \widehat{A}
\end{array}\right), \quad \widehat{A} \in \mathbb{C}^{(n-1) \times(n-1)}, \quad \operatorname{det} \widehat{A} \neq 0 .
$$


Due to the assumption (4), there is $i \in\{1, \ldots, p\}$ such that the column $b=\left(b_{1 i}, \ldots, b_{n i}\right)^{T}$ of the matrix $B$ possesses a nonzero first component: $b_{1 i} \neq 0$. Besides, since $\operatorname{det} \widehat{A} \neq 0$, then there is an unique $\alpha=$ $\left(\alpha_{2}, \ldots, \alpha_{n}\right)^{T} \in \mathbb{C}^{n-1}$ such that $\widehat{b}=\widehat{A} \alpha$, where $\widehat{b}=\left(b_{2 i}, \ldots, b_{n i}\right)^{T} \in \mathbb{C}^{n-1}$.

Let us analyze the structure of $\Delta\left(\lambda_{1}^{k}\right)$. We rewrite it as follows:

$$
\Delta\left(\lambda_{1}^{k}\right)=\lambda_{1}^{k} \mathrm{e}^{-\lambda_{1}^{k}}\left(A_{-1}-\mu_{1} I+D_{k}\right)
$$

where

$$
D_{k}=\left(\mathrm{e}^{\tilde{\lambda}_{1}^{k}}-\mathrm{e}^{\lambda_{1}^{k}}\right) I+\mathrm{e}^{\lambda_{1}^{k}} \int_{-1}^{0} \mathrm{e}^{\lambda_{1}^{k} \theta}\left(A_{2}(\theta)+\frac{1}{\lambda_{1}^{k}} A_{3}(\theta)\right) \mathrm{d} \theta .
$$

From the last formula, we conclude that $D_{k} \rightarrow 0$ when $k \rightarrow \infty$.

Let us show that there is $N \in \mathbb{N}$ such that for any $k:|k| \geq N$ :

$$
\operatorname{rank}\left(\Delta\left(\lambda_{1}^{k}\right), b\right)=n
$$

which is equivalent to the statement of the proposition.

Since $\operatorname{det} \Delta\left(\lambda_{1}^{k}\right)=0$, then $\operatorname{det}\left(A_{-1}-\mu_{1} I+D_{k}\right)=0$. Let us denote by $\widehat{D}_{k}=\left\{\left(D_{k}\right)_{i j}\right\}_{i, j=2}^{n}$. Since $\operatorname{det} \widehat{A} \neq 0$, then there is $N \in \mathbb{N}$ such that for any $k:|k| \geq N$ we have: $\operatorname{det}\left(\widehat{A}+\widehat{D}_{k}\right) \neq 0$. Therefore, there is a unique vector $\alpha_{k} \in \mathbb{C}^{n}$ such that $\widehat{b}=\left(\widehat{A}+\widehat{D}_{k}\right) \alpha_{k}$. From the latter, we conclude

$$
\alpha_{k}=\left(\widehat{A}+\widehat{D}_{k}\right)^{-1} \widehat{b}=\left(I+\widehat{A}^{-1} \widehat{D}_{k}\right)^{-1} \widehat{A}^{-1} \widehat{b}=\left(I+\widehat{A}^{-1} \widehat{D}_{k}\right)^{-1} \alpha .
$$

Since $\left(I+\widehat{A}^{-1} \widehat{D}_{k}\right)^{-1} \rightarrow I$ when $k \rightarrow \infty$, we conclude that $\alpha_{k} \rightarrow \alpha$.

If we suppose that (6.7) does not hold, then $b_{1}=b_{1 i}$ allows the following representation:

$$
b_{1 i}=\left(\alpha_{k}\right)_{2}\left(D_{k}\right)_{12}+\left(\alpha_{k}\right)_{n}\left(D_{k}\right)_{1 n} .
$$

However, since $\left(D_{k}\right)_{1 i} \rightarrow 0$, the right-hand side of the last relation tends to zero when $k \rightarrow \infty$. We have come to the contrary, which completes the proof of the proposition.

As mentioned above, for the sake of completeness we give the formulations of the results on the infinite pole assignment from [29]. We formulate them in a form which takes into account the specific form of the operator $\widehat{\mathcal{A}}_{1}$.

Theorem 6.5 (on infinite pole assignment [29]). Let $H$ be a complex Hilbert space, $\mathcal{A}$ be an infinitesimal generator of a $C_{0}$-semigroup in $H$, and the control system be given by $\dot{x}=\mathcal{A} x+\mathcal{B} u, x \in \mathcal{D}(\mathcal{A}) \subset H$. Let $\mu_{1}, \ldots, \mu_{\ell}$ be some nonzero complex numbers and we introduce the complex numbers

$$
\widetilde{\lambda}_{m}^{k}=\ln \left|\mu_{m}\right|+\mathrm{i}\left(\arg \mu_{m}+2 \pi k\right), \quad m=1, \ldots, \ell, \quad k \in \mathbb{Z},
$$

and the circles $L_{m}^{k}\left(r^{(k)}\right)$ centered at $\widetilde{\lambda}_{m}^{k}$ with radii $r^{(k)}$ satisfying the relation $\sum_{k \in \mathbb{Z}}\left(r^{(k)}\right)^{2}<\infty$.

Let the following assumptions hold:

(H1) The spectrum of $\mathcal{A}$ consists only of eigenvalues which are located in the circles $L_{m}^{k}\left({ }^{(k)}\right)$. Moreover, all the eigenvalues of $\mathcal{A}$ are simple (i.e. its algebraic multiplicity equals 1 ) and there is $N_{1} \in \mathbb{N}$ such that for any $k:|k| \geq N_{1}$ the total multiplicity of the eigenvalues contained in the circles $L_{m}^{k}\left(r^{(k)}\right)$ equals 1 .

(H2) The corresponding eigenvectors, which we denote by $\varphi_{m}^{k}$, constitute a Riesz basis in $H$.

(H3) The system $\dot{x}=\mathcal{A} x+\mathcal{B} u$ is of a single input, i.e. the operator $\mathcal{B}: \mathbb{C} \rightarrow H$ is the operator of multiplication by $\mathbf{b} \in H$. 
(H4) We assume the following controllability condition: $\mathbf{b}$ is not orthogonal to eigenvectors $\psi_{m}^{k}$ of the operator $\mathcal{A}^{*}:\left\langle\mathbf{b}, \psi_{m}^{k}\right\rangle \neq 0$ and

$$
\lim _{k \rightarrow \infty} k\left|\left\langle\mathbf{b}, \psi_{m}^{k}\right\rangle\right|=c_{m}, \quad 0<c_{m}<+\infty .
$$

Then there is $N_{2} \geq N_{1}$ such that for any family of complex numbers $\widehat{\lambda}_{m}^{k} \in L_{m}^{k}\left(r^{(k)}\right),|k| \geq N_{2}$ there is a linear control $\mathcal{F}: \mathcal{D}(\mathcal{A}) \rightarrow \mathbb{C}$, such that

(1) The complex numbers $\widehat{\lambda}_{m}^{k}$ are eigenvalues of the operator $\mathcal{A}+\mathcal{B F}$,

(2) The operator $\mathcal{B F}: \mathcal{D}(\mathcal{A}) \rightarrow H$ is relatively $\mathcal{A}$-bounded.

Lemma 6.6 ([29]). If the assumptions (3) and (4) of Theorem 6.1 hold, then there is a vector $b \in \operatorname{Im} B$, say $b=c_{1} b_{1}+\ldots+c_{p} b_{p}, c_{i} \in \mathbb{C}$, such that the following relations hold:

(a) $\left\langle b, y_{m}\right\rangle_{\mathbb{C}^{n}} \neq 0$ for all eigenvectors $y_{m}$ of the matrix $A_{-1}^{*}$ corresponding to the eigenvalues $\overline{\mu_{m}}$, where $\mu_{m} \in \sigma_{1}, m=1, \ldots, \ell_{1}$.

(b) $\left\langle b, y_{m}^{k}\right\rangle_{\mathbb{C}^{n}} \neq 0$ for all such $y_{m}^{k}$ which satisfy $y_{m}^{k} \in \operatorname{Ker} \Delta^{*}\left(\overline{\lambda_{m}^{k}}\right)$ and $\operatorname{Re} \lambda_{m}^{k} \geq 0$.

\section{Conclusion And Perspectives}

In the present paper, we have generalized the results on strong asymptotic non-exponential stability and regular stabilizability for the case of mixed retarded-neutral type systems. The proofs of these generalizations are technically complicated and require subtle estimates. We combine the Riesz basis technique with the analysis of the boundedness of the resolvent on some $\mathcal{A}$-invariant subspaces.

As a perspective, we consider systems with the difference operator $K$ given by

$$
K f=\sum_{i=1}^{r} A_{h_{i}} f\left(h_{i}\right), \quad h_{i} \in[-1,0] .
$$

Besides, the dilemma of item (iii) of Theorem 1.1 on stability may be investigated more precisely.

\section{REFERENCES}

[1] W. Arendt and C.J.K. Batty, Tauberian theorems and stability of one-parameter semigroups. Trans. Am. Math. Soc. 306 (1988) 837-852.

[2] A. Bátkai, K.-J. Engel, J. Prüss and R. Schnaubelt, Polynomial stability of operator semigroups. Math. Nachr. 279 (2006) $1425-1440$.

[3] R. Bellman and K.L. Cooke, Differential-difference equations. Academic Press, New York (1963).

[4] C. Bonnet, A.R. Fioravanti and J.R. Partington, On the stability of neutral linear systems with multiple commensurated delays, in IFAC Workshop on Control of Distributed Parameter Systems. Toulouse (2009) 195-196. IFAC/LAAS-CNRS.

[5] A. Borichev and Y. Tomilov, Optimal polynomial decay of functions and operator semigroups. Math. Ann. 347 (2010) 455-478.

[6] W.E. Brumley, On the asymptotic behavior of solutions of differential-difference equations of neutral type. J. Diff. Equ. 7 (1970) $175-188$.

[7] J.A. Burns, T.L. Herdman and H.W. Stech, Linear functional-differential equations as semigroups on product spaces. SIAM J. Math. Anal. 14 (1983) 98-116.

[8] R.F. Curtain and H. Zwart, An introduction to infinite-dimensional linear systems theory, Texts in Applied Mathematics 21. Springer-Verlag, New York (1995).

[9] X. Dusser and R. Rabah, On exponential stabilizability of linear neutral type systems. Math. Probl. Eng. 7 (2001) 67-86.

[10] J.K. Hale and S.M.V. Lunel, Introduction to functional-differential equations, Applied Mathematical Sciences 99. SpringerVerlag, New York (1993).

[11] J.K. Hale and S.M.V. Lunel, Strong stabilization of neutral functional differential equations. IMA J. Math. Control Inf. 19 (2002) 5-23. Special issue on analysis and design of delay and propagation systems.

[12] D. Henry, Linear autonomous neutral functional differential equations. J. Diff. Equ. 15 (1974) 106-128.

[13] C.A. Jacobson and C.N. Nett, Linear state-space systems in infinite-dimensional space: the role and characterization of joint stabilizability/detectability. IEEE Trans. Automat. Control 33 (1988) 541-549.

[14] T. Kato, Perturbation theory for linear operators, Die Grundlehren der mathematischen Wissenschaften, Band 132. SpringerVerlag New York, Inc., New York (1966). 
[15] V.B. Kolmanovskii and V.R. Nosov, Stability of functional-differential equations, Mathematics in Science and Engineering 180. Academic Press Inc. [Harcourt Brace Jovanovich Publishers], London (1986).

[16] L.A. Liusternik and V.J. Sobolev, Elements of functional analysis, Russian Monographs and Texts on Advanced Mathematics and Physics 5. Hindustan Publishing Corp., Delhi (1961).

[17] Z.-H. Luo, B.-Z. Guo and O. Morgul, Stability and stabilization of infinite dimensional systems with applications. Communications and Control Engineering Series, Springer-Verlag London Ltd., London (1999).

[18] Yu.I. Lyubich and V.Q. Phóng, Asymptotic stability of linear differential equations in Banach spaces. Studia Math. 88 (1988) $37-42$.

[19] S.A. Nefedov and F.A. Sholokhovich, A criterion for stabilizability of dynamic systems with finite-dimensional input. Differentsial' nye Uravneniya 22 (1986) 223-228, 364.

[20] D.A. O'Connor and T.J. Tarn, On stabilization by state feedback for neutral differential-difference equations. IEEE Trans. Automat. Control 28 (1983) 615-618.

[21] L. Pandolfi, Stabilization of neutral functional differential equations. J. Optim. Theory Appl. 20 (1976) 191-204.

[22] J.R. Partington and C. Bonnet, $H_{\infty}$ and BIBO stabilization of delay systems of neutral type. Syst. Control Lett. 52 (2004) 283-288.

[23] L.S. Pontryagin, On the zeros of some elementary transcendental functions. Amer. Math. Soc. Transl. 1 (1955) 95-110.

[24] R. Rabah and G.M. Sklyar, Strong stabilizability for a class of linear time delay systems of neutral type. Mat. Fiz. Anal. Geom. 11 (2004) 314-330.

[25] R. Rabah and G.M. Sklyar, On a class of strongly stabilizable systems of neutral type. Appl. Math. Lett. 18 (2005) $463-469$.

[26] R. Rabah and G.M. Sklyar, The analysis of exact controllability of neutral-type systems by the moment problem approach. SIAM J. Control Optim. 46 (2007) 2148-2181.

[27] R. Rabah, G.M. Sklyar and A.V. Rezounenko, Generalized Riesz basis property in the analysis of neutral type systems. $C$. $R$. Math. Acad. Sci. Paris 337 (2003) 19-24.

[28] R. Rabah, G.M. Sklyar and A.V. Rezounenko, Stability analysis of neutral type systems in Hilbert space. J. Diff. Equ. 214 (2005) 391-428.

[29] R. Rabah, G.M. Sklyar and A.V. Rezounenko, On strong regular stabilizability for linear neutral type systems. J. Diff. Equ. 245 (2008) 569-593.

[30] G.M. Sklyar, Lack of maximal asymptotics for some linear equations in a Banach space. Dokl. Math. 81 (2010) $265-267$. Extended version to appear in Taiwanese Journal of Mathematics (2011).

[31] G.M. Sklyar and A.V. Rezounenko, Stability of a strongly stabilizing control for systems with a skew-adjoint operator in Hilbert space. J. Math. Anal. Appl. 254 (2001) 1-11.

[32] G.M. Sklyar and A.V. Rezounenko, A theorem on the strong asymptotic stability and determination of stabilizing controls. $C$. R. Acad. Sci. Paris Sér. I Math. 333 (2001) 807-812.

[33] G.M. Sklyar and V.Ya. Shirman, On asymptotic stability of linear differential equation in Banach space. Teor. Funktsǐ Funktsional. Anal. i Prilozhen. 37 (1982) 127-132.

[34] R. Triggiani, On the stabilizability problem in Banach space. J. Math. Anal. Appl. 52 (1975) 383-403.

[35] J. van Neerven, The asymptotic behaviour of semigroups of linear operators, Operator Theory: Advances and Applications 88. Birkhäuser Verlag, Basel (1996).

[36] S.M. Verduyn Lunel and D.V. Yakubovich, A functional model approach to linear neutral functional-differential equations. Integr. Equ. Oper. Theory 27 (1997) 347-378.

[37] V.V. Vlasov, Spectral problems that arise in the theory of differential equations with delay. Sovrem. Mat. Fundam. Napravl. 1 (2003) 69-83 (electronic).

[38] W.M. Wonham, Linear multivariable control, Applications of Mathematics 10. 3th edition, Springer-Verlag, New York (1985). 